REVIEWS OF INFECTIOUS DISEASES - VOL. 12, NO. 1 - JANUARY-FEBRUARY 1990

(C) 1990 by The University of Chicago. All rights reserved. $0612-0882 / 90 / 1201-0029 \$ 02.00$

\title{
Global Genetic Structure and Molecular Epidemiology of Encapsulated Haemophilus influenzae
}

James M. Musser; J. Simon Kroll, Dan M. Granoff, E. Richard Moxon, Bernard R. Brodeur, Jose Campos, Henri Dabernat, Wilhelm Frederiksen, Josee Hamel, Gregory Hammond, E. Arne Hoiby, Kristin E. Jonsdottir, Mustafa Kabeer, Ingegerd Kallings, Waheed N. Khan, Mogens Kilian, Kathleen Knowles, H. J. Koornhof, Barbara Law, Karl I. Li, Janet Montgomery, Patricia E. Pattison, Jean-Claude Piffaretti, Aino K. Takala, Mee Len Thong, Robert A. Wall, Joel I. Ward, and Robert K. Selander
From the Department of Biology, Mueller Laboratory, Pennyslvania State University, University Park; Infectious Disease Unit, Department of Paediatrics, John Radcliffe Hospital, Headington, Oxford; Edward Mallinckrodt Department of Pediatrics, Washington University School of Medicine, St. Louis; Hybridoma Section, Bureau of Microbiology, Laboratory Centre for Disease Control, Health and Welfare Canada, Ottawa; Departments of Microbiology and Pediatrics, Hospital Infantil San Juan de Dios, Barcelona; Laboratoire Central de Microbiologie, C. H. U. Toulouse Purpan, Toulouse; Statens Seruminstitut, Copenhagen; Cadham Provincial Laboratory, Winnipeg; National Institute of Public Health, Oslo; Department of Bacteriology, University of Iceland, Reykjavik; The National Bacteriology Laboratory, Stockholm; Children's Hospital National Medical Center, Washington, D.C.; Department of Oral Biology, Royal Dental College, Aarhus; Montreal Children's Hospital, Montreal; South African Institute for

Medical Reseanch, Johannesburg; Department of Pediatrics and Child Health, University of Manitoba, Winnipeg; Children's Hospital of Pittsburgh, Pennsylvania; Papua New Guinea Institute of Medical Research, Goroka; Instituto Cantonale Batteriosierologico, Lugano; National Public Health Institute, Helsinki; Division of Microbiology, Department of Pathology, North Brisbane Hospitals Board, Brisbane; Medical Research Council Laboratories, Faraja; Department of Pediatrics, Harbor-UCLA Medical Center, University of California, Los Angeles

A collection of 2,209 isolates of six polysaccharide capsule types of Haemophilus influen$z a e$, including 1,975 serotype $b$ isolates recovered in 30 countries was characterized for electrophoretically demonstrable allele profiles at 17 metabolic enzyme loci. Two hundred eighty distinct multilocus genotypes were distinguished, and cluster analysis revealed two primary phylogenetic divisions. The population structure of encapsulated $H$. influenzae is clonal. Currently, most of the invasive disease worldwide is caused by serotype $b$ strains of nine clones. Strains producing serotype $c$, $e$, and $f$ capsules belong to single divisions and have no close genetic relationships to strains of other serotypes. Serotype $a$ and $b$ strains occur in both primary phylogenetic divisions, probably as a result of transfer and recombination of serotype-specific sequences of the cap region between clonal lineages. A close genetic relatedness between serotype $d$ isolates and some strains of serotypes a and $b$ was identified. There are strong patterns of geographic variation, on an intercontinental scale, in both the extent of genetic diversity and the clonal composition of populations of encapsulated strains. The analysis suggests that the present distribution of clones is, in part, related to patterns of racial or ethnic differentiation and historical demographic movements of the human host populations.

Received for publication 22 February 1989 and in revised form 13 June 1989.

Research leading to this review was supported by grant no. AI24332 from the National Institute of Allergy and Infectious Diseases (to R. K. S. and D. M. G.), by contract AID-2429-8-312 from the Board on Science and Technology for International Development (BOSTID), National Research Council (to D. M. G.), by a Program Grant from the Medical Research Council, U.K. (to E. R. M.), and by grant 85.3.16.8.E from the Caisse Nationale d'Assurances Maladie des Travailleurs Salaries (to H. D.). J. S. K. is a Lister Institute Research Fellow.
This paper is dedicated to Dr. Margaret Pittman.

C. M. Sommers and L. M. Tremblay provided technical assistance. The authors thank T. S. Whittam for help with data analysis. The senior author thanks K. A. Zeznick for extensive encouragement.

* Present affiliation: Department of Pathology and Laboratory Medicine, Hospital of the University of Pennsylvania, Philadelphia, Pennsylvania.

Please address request for reprints to Dr. Robert K. Selander, Department of Biology, Mueller Laboratory, Pennsylvania State University, University Park, Pennsylvania 16802. 
Encapsulated strains of Haemophilus influenzae are a major cause of meningitis and other serious invasive diseases, including septicemia, obstructive epiglottitis, cellulitis, and septic arthritis, in young children in many parts of the world [1-3]. Because of their great public health importance, encapsulated strains, especially those expressing the serotype $b$ polysaccharide, have been the subject of intensive research in the United States and Europe during the past 10 years. Early immunologic and metabolic studies demonstrated heterogeneity among strains [4-6], but attempts to understand the epidemiology of disease caused by encapsulated organisms initially were hindered by the lack of high-resolution, stable genetic marker systems for strain discrimination and classification.

Early in the present decade, techniques were developed for classifying serotype $b$ isolates on the basis of variation in the electrophoretic mobility patterns of the major outer-membrane proteins (OMPs) [7-10] and lipopolysaccharide [11, 12], serologic diversity in lipopolysaccharide antigens $[9,13,14]$, and other phenotypic characters [15-17]. An examination of 51 serotype $b$ isolates recovered from children in St. Louis with invasive infections identified nine distinctive OMP pattern subtypes and demonstrated that five subtypes, designated as $1 \mathrm{H}, 1 \mathrm{~L}, 2 \mathrm{H}$, $2 \mathrm{~L}$, and $3 \mathrm{~L}$, accounted for $92 \%$ of the strains [18]. In a survey of 256 invasive isolates from a variety of clinical settings in 22 states in the United States, Granoff et al. [19] distinguished 21 OMP subtypes and discovered that about $70 \%$ of cases were caused by strains of three subtypes $(1 \mathrm{H}, 2 \mathrm{~L}$, and $3 \mathrm{~L})$. Subsequently, van Alphen et al. [9] examined 80 isolates from invasive infections in the Netherlands where, in striking contrast to those in the United States, $\mathbf{8 4 \%}$ of isolates had the same OMP pattern (type 1; subtype 3L in the Granoff laboratory system); moreover, no strains of $1 \mathrm{H}, 1 \mathrm{~L}$, or $2 \mathrm{H}$ were identified.

In an effort to measure genetic diversity and evolutionary genomic relationships among serotype $b$ isolates classified by OMP type and biotype, Musser et al. [20] analyzed genetically determined electrophoretic variation in 16 metabolic enzymes in 177 isolates from the United States. They identified 32 distinctive multilocus enzyme genotypes (electrophoretic types, ETs) [20] and determined that $73 \%$ of invasive disease episodes were caused by strains of only three ETs. This work also demonstrated that serotype $b$ capsule is expressed in strains belonging to four rather distantly related groups of chromosomal genotypes, each of which is associated with a characteristic set of OMP patterns [20]. To explain the occurrence of strong nonrandom associations of multilocus enzyme genotype, OMP subtype, and biotype, and the repeated recovery of isolates with identical properties in widely separated geographic regions and over a 40-year period, researchers hypothesized [20] and subsequently confirmed [21, 22] that the structure of natural populations of serotype $\mathrm{b} H$. influenzae is basically clonal as a consequence of infrequent recombination of chromosomal genes.

Two important characteristics of the epidemiology of serotype b $H$. influenzae were identified in these studies. First, although there is extensive genetic diversity among strains expressing type $b$ capsule, most disease is caused by a very small number of clonal chromosomal genotypes, which are marked by ETs. Second, comparison of results from studies in the United States and the Netherlands suggested the existence of strong patterns of geographic variation, on an intercontinental scale, in both the extent of genetic diversity and the clonal composition of populations.

Little is known about the genetics of populations of $H$. influenzae in areas of the world other than the United States and western Europe [23]. To study genetic structure and molecular epidemiology on a global scale, we assembled a large collection of encapsulated $\boldsymbol{H}$. influenzae strains recovered in many countries over the past 40 years. One objective was to generate baseline data that would permit prospective assessment of the effects of vaccination on the genetic diversity and structure of populations of invasive and carrier strains. Additionally, we wanted to examine possible correlations between patterns of geographic variation in genetic diversity and clonal composition of the serotype $b$ populations and patterns of human racial/ethnic differentiation and historical demographic movement. For immunoprophylactic research, development, and application, it may be important to understand the global clone distribution of type $b$ strains.

We here present a comprehensive analysis of the genetic structure and molecular epidemiology of encapsulated $H$. influenzae, based on 2,209 isolates from six continents. The population genetic analysis is based primarily on electrophoresis of 17 chromosomally encoded metabolic enzymes, with additional information being supplied by OMP subtyp- 
ing $[8,24,25]$ and the restriction fragment length polymorphism (RFLP) pattern of the cap region $[24,26]$.

\section{Materials and Methods}

\section{Bacterial Isolates}

A collection of 2,209 isolates of encapsulated $H$. influenzae recovered from individuals in $\mathbf{3 0}$ countries on six continents was examined (table 1). The sample includes 52 isolates of serotype a, 1,975 of serotype b, 13 of serotype c, 27 of serotype d, 92 of serotype $e$, and 50 of serotype $f$. Of the serotype b strains, 1,814 were cultured from blood, cerebrospinal fluid, or other normally sterile body fluid, 91 from the nasopharynx of healthy individuals, 54 from severe lower respiratory tract infections (predominantly in Malaysia), and six from the eyes of patients with conjunctivitis. Most isolates of other serotypes were recovered from asymptomatic carriers or from patients with surface infections. However, 11 isolates of serotype a, six of serotype $f$, and two each of serotypes $\mathrm{d}$ and $\mathrm{e}$ were cultured from patients with invasive infections. The collection includes 24 isolates obtained between 1939 and 1954 [27], but most of the isolates were recovered between 1965 and 1987.

Of the carrier isolates, 46 were recovered from associated children in three day care centers in Spain [28], 10 were from Amerinds living on the Fort Apache Indian Reservation in Arizona, 20 were from Alaskan Natives in a variety of areas in the state, nine were from associated South Korean children taken to Norway for adoption, and six were from unknown sources in the United States and Canada.

Most isolates were obtained from a collection assembled by the senior author and from collections maintained by the coauthors. In addition, the following individuals each supplied a small number of isolates: J. O. Achola, University of Nairobi, Kenya; M. Arpi, Statens Seruminstitut, Copenhagen, Denmark; M. Catalano, National Research Council, Buenos Aires, Argentina; B. W. Catlin, Medical College of Wisconsin, Milwaukee, Wisconsin; P. D. Ellner, Columbia-Presbyterian Medical Center, New York, New York; R. J. Fallon, Ruchill Hospital, Glasgow, Scotland; P. Fleming, Hospital for Sick Children, Toronto, Ontario, Canada; E. J. Hansen, University of Texas Health Science Center, Dallas, Texas; H. Heffernan, National Health Institute, Welling- ton, New Zealand; T. Konda, National Institute of Health, Tokyo, Japan; J. Levy, Rochester General Hospital, Rochester, New York; M. Loeb, University of Rochester School of Medicine and Dentistry, Rochester, New York; M. Magdasy, Central Public Health Laboratory, Montevideo, Uruguay; K. McGowan, St. Christopher's Hospital, Philadelphia, Pennsylvania; T. Oguri, Juntendo University, Tokyo, Japan; S. J. Oppenheimer, Hospital Universiti Sains Malaysia, Kelantan, Malaysia; T. H. Pennington, University of Aberdeen, Scotland; C. A. Reichart, Johns Hopkins Medical Institutions, Baltimore, Maryland; S. H. Sell, Vanderbilt University Medical School, Nashville, Tennessee; A. L. Smith, Children's Orthopedic Hospital and Medical Center, Seattle, Washington; M. Soraekit, Mahidal University, Thailand; J. Spainhour, Children's Hospital of Philadelphia, Philadelphia, Pennyslvania; Y. Terawaki, Shinshu University School of Medicine, Matsumoto, Japan; T. Tupasi, Makati Medical Center, Philippines; M. K. Wagner, Kapiolani Women's and Children's Medical Center, Honolulu, Hawaii; L. B. Weiner, Upstate Medical Center, Syracuse, New York; and R. Yogev, Children's Memorial Hospital, Chicago, Illinois.

\section{Electrophoresis of Enzymes}

Isolates were grown overnight at $37^{\circ} \mathrm{C}$ in $150 \mathrm{~mL}$ of brain-heart infusion broth (Difco; Detroit, Mich.), supplemented with NAD and hemin, each at $2 \mathrm{mg}$ / $\mathrm{mL}$. Cells were harvested by centrifugation, suspended in $2 \mathrm{~mL}$ of $50 \mathrm{mM}$ Tris-hydrochloride, $\mathrm{pH}$ 8.0, containing $5 \mathrm{mM}$ EDTA, and sonicated (Branson Sonifier Cell Disruptor, model 200, with microtip; Danbury, Conn.) for 30 seconds at $50 \%$ pulse, with ice-bath cooling. After centrifugation at 20,000 $\times g$ for 20 minutes at $4^{\circ} \mathrm{C}$, the clear supernatant (lysate) was stored at $-70^{\circ} \mathrm{C}$.

Techniques of horizontal starch-gel electrophoresis and the demonstration of specific enzyme activity were similar to those described elsewhere [20,29, 30]. The 17 enzymes assayed were carbamylate kinase (CAK), nucleoside phosphorylase (NSP), phosphoglucose isomerase (PGI), malic enzyme (MAE), malate dehydrogenase (MDH), glucose-6-phosphate dehydrogenase (G6P), glutamic oxaloacetic transaminase (GOT), adenylate kinase (ADK), 6-phosphogluconate dehydrogenase (6PG), leucylalanine peptidase 1 (PE1), leucylalanine peptidase 2 (PE2), 
Table 1. Composition of the sample of encapsulated $H$. influenzae, grouped by geographic source.

\begin{tabular}{|c|c|c|c|c|c|c|c|c|c|c|c|}
\hline \multirow[b]{3}{*}{ Geographic source } & \multirow{3}{*}{$\begin{array}{l}\text { Collection } \\
\text { period }\end{array}$} & \multicolumn{2}{|c|}{$\begin{array}{l}\text { No. of } \\
\text { isolates }\end{array}$} & \multicolumn{2}{|c|}{$\begin{array}{l}\text { No. of } \\
\text { ETs }\end{array}$} & \multirow[b]{3}{*}{ Geographic source } & \multirow{3}{*}{$\begin{array}{l}\text { Collection } \\
\text { period }\end{array}$} & \multicolumn{2}{|c|}{$\begin{array}{l}\text { No. of } \\
\text { isolates }\end{array}$} & \multicolumn{2}{|c|}{$\begin{array}{l}\text { No. of } \\
\text { ETs }\end{array}$} \\
\hline & & $\begin{array}{l}\text { Sero- } \\
\text { type }\end{array}$ & & $\begin{array}{l}\text { Sero- } \\
\text { type }\end{array}$ & & & & $\begin{array}{l}\text { Sero- } \\
\text { type }\end{array}$ & & $\begin{array}{l}\text { Sero- } \\
\text { type }\end{array}$ & \\
\hline & & b & Other & $\mathrm{b}$ & Other & & & b & Other & $\mathrm{b}$ & Other \\
\hline North America & & 936 & & 104 & & Africa & & 93 & & 27 & \\
\hline \multirow{3}{*}{$\begin{array}{l}\text { Canada } \\
\text { United States }\end{array}$} & $1969-1986$ & $376^{a}$ & & 40 & & The Gambia & $1983-1984$ & $30^{\mathrm{m}}$ & 5 & 6 & 2 \\
\hline & 1939-1954, & & & & & Ghana & 1983-1984 & 5 & & 3 & \\
\hline & $1968-1987$ & $560^{\mathrm{b}}$ & 12 & 77 & 10 & Kenya & $1980 \mathrm{~s}$ & 10 & 9 & 4 & 8 \\
\hline \multicolumn{2}{|l|}{ Europe } & 623 & & 60 & & Rep. of South Africa & $1984-1986$ & $48 n$ & & 18 & \\
\hline \multirow[t]{2}{*}{ Denmark $^{c}$} & 1940-1942, & & & & & Papua New Guinea & $1980-1985$ & 60 & 4 & 9 & 4 \\
\hline & $1980-1986$ & 46 & & 18 & & Other & & 143 & & 29 & \\
\hline England & $1983-1985$ & 21 & 149 & 8 & 52 & Argentina & 1986 & $4^{o}$ & & 2 & \\
\hline Finland & 1985 & $100^{d}$ & & 12 & & Australia & $1984-1986$ & $40 p$ & & 10 & \\
\hline France & $1980 \mathrm{~s}$ & $77^{\mathrm{e}}$ & & 10 & & Guatemala & 1986 & 1 & & 1 & \\
\hline Iceland & $1977-1986$ & $40^{f}$ & & 6 & & Hawaii & $1985-1986$ & 22 & & 11 & \\
\hline The Netherlands & $1975-1982$ & 8 & & 4 & & Mexico & 1986 & 19 & & 1 & \\
\hline Norway & $1980-1985$ & 39 & & 11 & & New Zealand & $1985-1986$ & $19 r$ & & 5 & \\
\hline Scotland & $1983-1986$ & 20 & & 7 & & Dominican Republic & 1980 & 55 & 2 & 14 & 2 \\
\hline Spain & $1980 \mathrm{~s}$ & 718 & & 3 & & Uruguay & 1986 & $1^{\mathrm{s}}$ & & 1 & \\
\hline Sweden & $1982-1986$ & $78^{h}$ & & 13 & & Unknown & $\ldots$ & & 14 & & 8 \\
\hline Switzerland & $1982-1986$ & $123^{i}$ & & 18 & & & & & & & \\
\hline Asia & & 120 & & 17 & & Total & & 1,975 & 234 & 182 & 98 \\
\hline Japan & $1981-1985$ & $12^{j}$ & & 3 & & & & & & & \\
\hline Malaysia & $1971-1979$ & $80^{k}$ & 39 & 11 & 19 & & & & & & \\
\hline Philippines & 1986 & 9 & & 4 & & & & & & & \\
\hline South Korea & 1985 & 91 & & 3 & & & & & & & \\
\hline Thailand & 1986 & 10 & & 4 & & & & & & & \\
\hline
\end{tabular}

a Alberta (26), Ontario (81), Quebec (84), Manitoba (145), New Brunswick (6), Prince Edward Island (26), and British Columbia (8).

b Alabama (1), Alaska (88), Arizona (34), California (1), Colorado (2), Florida (1), Illinois (11), Indiana (1), Louisiana (1), Maryland (31), Massachusetts (1), Minnesota (45), Missouri (7), New York (78), North Carolina (2), Oklahoma (52), Pennsylvania (136), Rhode Island (1), Tennessee (13), Texas (27), Washington, D.C. (1), Washington state (10), Wisconsin (16), Hawaii excluded.

c Includes Greenland.

d Espoo (1), Helsinki (19), Joensuu (9), Jyvaskyla (6), Kajaani (1), Kemi (1), Kokkola (3), Kotka (2), Kuopio (9), Lappeenranta (3), Oulu (18), Pori (3), Porvoo (1), Rovaniemi (2), Savonlinna (2), Tammisaari (2), Turku (7), EPKS (6), PHKS (2), VAKS (1), and unknown (2). e Aix en Provence (3), Bordeux (2), Clermont Ferrand (3), Colombes (1), Compiegne (2), Creteil (10), Le Mans (2), Longjumeau (2), Mulhouse (2), Nantes (2), Nice (2), Reims (5), Rennes (3), Strasbourg (11), Toulouse (21), Versailles (4), and Villeneuve Saint Georges (2).

f Akureyri (3), Breiddalsvik (1), Budardalur (1), Djupivogur (1), Egilsstadir (1), Eskifjordur (1), Gardabaer (1), Hafnarfjordur (1), Husavik

(1), Keflavik (2), Keflavik (U.S.) naval base (1), Kopavogur (2), Reykjavik (23), and Vestmannaeyjar (1).

B Barcelona area (47), Catalonia (18), northern Spain (3), southern Spain (3).

h Boden (1), Eskilstuna (4), Galve (1), Jonkoping (28), Kalmar (2), Karlskrona (1), Linkoping (2), Norrkoping (2), Skovde (3), Stockholm (19), Umea (1), Vasteras (4), Vastervik (2), Vaxdo (2), Visby (3), and unknown (3).

i Bern (24), Geneva (55), Lausanne (15), Lugano (1), San Gall (16), Winterthur (2), Zurich (10).

j Matsumoto (7), Tokyo (5).

$k$ Kuala Lumpur.

' Isolated from nose or throat of adopted South Korean children minutes after arrival at Fornebu Airport, Oslo, Norway.

m Many areas throughout the country.

n Cape Town (7), Durban (2), Johannesburg (32), unknown (7).

${ }^{\circ}$ Buenos Aires.

P Brisbane (38), Melbourne (2).

q Mexico City.

${ }^{r}$ Auckland (7), Christchurch (1), Hastings (4), Nelson (1), Palmerston North (2), Taumarunui (1), Wellington (2), Whakatane (1). $s$ Montevideo. 
leucine aminopeptidase (LAP), phosphoglucomutase (PGM), catalase (CAT), glutamate dehydrogenase (GLD), glyceraldehyde-3-phosphate dehydrogenase (G3P), and fumarase (FUM).

Electromorphs (allozymes) of each enzyme were equated with alleles at the corresponding structural gene locus, and distinctive combinations of alleles over the 17 enzyme loci, representing multilocus chromosomal genotypes, were designated as electrophoretic types (ETs) [30].

\section{Serotyping}

Serotypes were determined by slide agglutination with serotype-specific sera or by the antiserum agar method. Strains of serotype b that were of ETs not previously identified $[20,29]$ were reserotyped in the laboratory of D. M. G. by countercurrent immunoelectrophoresis with specific type $b$ rabbit antisera, prepared under contract for the Institute of Medicine. Isolates with multilocus enzyme genotypes that were very different from those of other isolates of the same serotype were reserotyped and tested for cap region pattern in the laboratory of E. R. M.

\section{Electrophoresis of OMPs}

Isolates of serotype a were typed previously for OMP pattern by Allan et al. [25]. Some serotype $b$ isolates also were typed previously [24] and the OMP patterns of additional serotype $b$ isolates were determined in the laboratories of J. I. W., E. R. M., and K. I. L. by the methods of Barenkamp et al. [8] or Loeb et al. [7], with standards supplied by D. M. G. In brief, electrophoresis was performed on detergentsoluble outer-membrane derivatives in an 8\%-17.5\% Laemmli linear gradient polyacrylamide gel system [31]. The serotype a isolates were assigned to categories on the basis of the gradient gel results and were further classified as $\mathrm{H}, \mathrm{L}$, or $\mathrm{U}$, depending upon the electrophoretic mobility of a heat-modifiable protein $(\mathrm{P} 1$, with an apparent molecular mass of $\sim 45,000$ [25]) on $11 \%$ acrylamide gels [32]. For serotype b strains, the heat-modifiable protein $\mathrm{P} 1$ has three electrophoretic variants, designated $H$ (50 $\mathrm{kDa}), \mathrm{L}(49 \mathrm{kDa})$, and $\mathrm{U}(<49 \mathrm{kDa})$, based on mobility in the Laemmli gel system. Because a detailed comparison of the OMP patterns of serotype $a$ and serotype b strains was not made, pattern designations are not necessarily cognate between serotypes.

\section{RFLP Analysis of the cap Region}

Isolates were typed for cap region RFLP pattern by the methods described elsewhere [24, 26]. Briefly, chromosomal DNA was digested with restriction endonuclease EcoRI, and fragments were resolved according to size on agarose gels, transferred to nitrocellulose filters, and probed with radiolabeled pUO38, a cloned fragment of serotype $\mathrm{b} H$. influenzae chromosomal DNA carrying genes involved in capsule synthesis [33]. The hybridization pattern was visualized by autoradiography. Strains were selected to include ETs representing the breadth of genotypic diversity and geographic origin in each of the major lineages of encapsulated $H$. influenzae.

\section{Statistical Analysis}

Genetic diversity at an enzyme locus among ETs or isolates was calculated as $h=\left(1-\Sigma x_{i}^{2}\right)[n /(n-1)]$, where $x_{i}$ is the frequency of the th allele and $n$ is the number of ETs or isolates in a sample [34]. Mean genetic diversity $(H)$ is the arithmetic average of $h$ values for all loci.

Genetic distance between pairs of ETs was expressed as the proportion of enzyme loci at which different alleles were represented (mismatches), and clustering of ETs was performed from a matrix of pairwise genetic distances by the average-linkage method [30,35].

\section{Results \\ Genetic and Genotypic Diversity}

All 17 enzyme loci assayed were polymorphic for from three (G3P) to 11 (PGI) alleles encoding electrophoretically distinctive variants, with an average of 6.4 alleles per locus. The failure of extracts of some isolates to show activity for one or two enzymes was attributed to the presence of null alleles, the highest frequency of which occurred at the NSP locus $(2.8 \%$ of the isolates). A total of 280 distinctive multilocus genotypes (ETs) was identified (table 2), among which mean genetic diversity per locus $(H)$ was 0.467 (table 3). Most ETs (63\%) were represented by single isolates, but 103 ETs (37\%) had multiple isolates (range, 2-497 isolates). The number of ETs per serotype ranged from seven for serotype $d$ isolates to 182 for serotype $b$ isolates. There was no sharing of ETs between serotypes. 
Table 2. Allele profiles and representative isolates of $280 \mathrm{ETs}$ of encapsulated $H$. influenzae.

\begin{tabular}{|c|c|c|c|c|c|c|c|c|c|c|c|c|c|c|c|c|c|c|c|c|c|c|c|c|c|}
\hline \multirow[b]{2}{*}{ Group } & \multirow{2}{*}{\multicolumn{2}{|c|}{$\begin{array}{cc} & \text { MHAEM } \\
\text { ET } & \text { no.* }\end{array}$}} & & & & & & & Allele a & at i & icated e & enzym & ne locu: & & & & & & & & OMP & & & & \\
\hline & & & CAK 1 & NSP & PGI 1 & MAE & MDH & G6P & GOT & ADK & $6 \mathrm{PG}$ & PE2 & PE1 & LAP & PGM & CAT & GLD & G3P & FUM & type & type & pattern & source & isolation & Other designation \\
\hline Ala & 1.9 & 712 & 9 & 5 & 1 & 5 & 5 & 3 & 5 & 2 & 2 & 4 & 5 & 2 & 4 & 2 & 4 & 2 & 2 & b & $1 \mathrm{H}$ & G & United States & 1980 & SLCH 668 \\
\hline Ala & 1.9 & 513 & 9 & 5 & 1 & 5 & 5 & 3 & 5 & 2 & 2 & 4 & 5 & 2 & 4 & 2 & 4 & 2 & 2 & $\mathrm{~b}$ & $2 \mathrm{~L}$ & $\mathrm{v}$ & United States & 1982 & RM1130 \\
\hline Ala & 1.9 & 1769 & 9 & 5 & 1 & 5 & 5 & 3 & 5 & 2 & 2 & 4 & 5 & 2 & 4 & 2 & 4 & 2 & 2 & b & $2 \mathrm{~L}$ & $s$ & Iceland & 1984 & B-7184 \\
\hline Ala & 2 & 1847 & 9 & 5 & 1 & 5 & 5 & 3 & 3 & 2 & 2 & 4 & 5 & 2 & 4 & 2 & 4 & 2 & 2 & b & IH & & Canada & $1980 \mathrm{~s}$ & LAW 10 \\
\hline Ala & 3 & 2910 & 9 & 5 & 1 & 5 & 5 & 3 & 1 & 2 & 2 & 4 & 5 & 2 & 4 & 2 & 4 & 2 & 2 & b & & & United States & & LOEB $\mathbf{S 1 3 8}$ \\
\hline Ala & 4 & 2368 & 9 & 5 & 1 & 5 & 5 & 3 & 6 & 2 & 2 & 4 & 5 & 2 & 4 & 2 & 4 & 2 & 2 & b & IL & & United States & $1980 \mathrm{~s}$ & HARBOR 117 \\
\hline Ala & 5 & 1081 & 9 & 5 & 1 & 5 & 5 & 3 & 5 & 2 & 4 & 4 & 5 & 2 & 4 & 2 & 4 & 2 & 2 & b & $1 \mathrm{H}$ & G & United States & $1970 \mathrm{~s}$ & SLCH 1338 \\
\hline Ala & 6 & 1065 & 9 & 5 & 1 & 5 & 5 & 3 & 6 & 2 & 4 & 4 & 5 & 2 & 4 & 2 & 4 & 2 & 2 & b & IL & G & United States & $1950 \mathrm{~s}$ & EAGAN \\
\hline Ala & 7 & 2383 & 9 & 5 & 1 & 5 & 5 & 3 & 6 & 2 & 3 & 4 & 5 & 2 & 4 & 2 & 4 & 2 & 2 & b & IL & & United States & $1980 \mathrm{~s}$ & HARBOR 185 \\
\hline Ala & 1.6 & 3078 & 6 & 5 & $i$ & 5 & 5 & 3 & 5 & 2 & 2 & 4 & 5 & 2 & 4 & 2 & 4 & 2 & 2 & b & $2 \mathrm{~L}$ & & Alaska & $1980 \mathrm{~s}$ & JW 32 \\
\hline Ala & 1.7 & 725 & 7 & 5 & 1 & 5 & 5 & 3 & 5 & 2 & 2 & 4 & 5 & 2 & 4 & 2 & 4 & 2 & 2 & b & & $\mathrm{v}$ & Australia & 1985 & RM7118 \\
\hline Ala & 1.8 & 3079 & 8 & 5 & 1 & 5 & 5 & 3 & 5 & 2 & 2 & 4 & 5 & 2 & 4 & 2 & 4 & 2 & 2 & b & IL & & Norway & $1980 \mathrm{~s}$ & D 143 \\
\hline Ala & 8 & 1811 & 8 & 5 & 1 & 5 & 5 & 3 & 6 & 2 & 2 & 4 & 5 & 2 & 4 & 2 & 4 & 2 & 2 & b & 3L & $\mathrm{s}$ & Kenya & $1980 \mathrm{~s}$ & RM7430 \\
\hline Ala & 9 & 1992 & 9 & 5 & 1 & 5 & 5 & 3 & 5 & 3 & 2 & 4 & 5 & 2 & 4 & 2 & 4 & 2 & 2 & $\mathrm{~b}$ & & & Canada & $1980 \mathrm{~s}$ & LAW 155 \\
\hline Ala & 10 & 3081 & 9 & 5 & 1 & 5 & 5 & 3 & 5 & 2 & 2 & 4 & 5 & 2 & 6 & 2 & 4 & 2 & 2 & b & & & United States & 1983 & RGH 4649 \\
\hline Ala & 11 & 1074 & 9 & 5 & 1 & 5 & 5 & 1 & 5 & 2 & 2 & 4 & 5 & 2 & 4 & 2 & 4 & 2 & 2 & b & IL & & United States & & SLCH 3279 \\
\hline $\mathrm{Ala}$ & 13 & 2949 & 9 & 5 & 1 & 5 & 5 & 6 & 5 & 2 & 2 & 4 & 5 & 2 & 4 & 2 & 4 & 2 & 2 & b & $1 \mathrm{H}$ & & United States & 1986 & SLCH $10,026 \mathrm{~A}$ \\
\hline Ala & 14 & 3080 & 9 & 5 & 1 & 5 & 5 & 3 & 5 & 2 & 2 & 4 & 5 & 2 & 4 & 3 & 4 & 2 & 2 & b & $1 \mathbf{H}$ & & Alaskia & $1980 \mathrm{~s}$ & JW 5 \\
\hline Ala & 15 & 3077 & 9 & 5 & 1 & 5 & 2 & 3 & 5 & 2 & 2 & 4 & 5 & 2 & 4 & 2 & 4 & 2 & 2 & b & $2 \mathrm{~L}$ & & United States & 1985 & PITT 8524 \\
\hline Ala & 16 & 1253 & 9 & 5 & 1 & 5 & 3 & 3 & 5 & 2 & 2 & 4 & 5 & 2 & 4 & 2 & 4 & 2 & 2 & b & & & Canada & $1980_{\mathrm{s}}$ & PERRIER \\
\hline Ala & 17 & 1982 & 9 & 5 & 1 & 5 & 5 & 3 & 5 & 2 & 2 & 4 & 2 & 2 & 4 & 2 & 4 & 2 & 2 & b & & & Canada & $1980 \mathrm{~s}$ & LAW 145 \\
\hline $\mathrm{Ala}$ & 18 & 3082 & 9 & 5 & 1 & 3 & 5 & 3 & 5 & 2 & 2 & 4 & 5 & 6 & 4 & 2 & 4 & 2 & 2 & b & $2 \mathrm{~L}$ & & Alaska & $1980 \mathrm{~s}$ & JW 11 \\
\hline Ala & 19 & 3083 & 9 & 5 & 1 & 3 & 5 & 3 & 5 & 2 & 2 & 4 & 5 & 2 & 4 & 2 & 4 & 2 & 2 & b & $2 \mathrm{~L}$ & & Alaska & $1980 \mathrm{~s}$ & JW 36 \\
\hline Ala & 20 & 3732 & 9 & 5 & 1 & 5 & 5 & 3 & 5 & 2 & 2 & 4 & 5 & 6 & 4 & 2 & 4 & 2 & 2 & b & $1 \mathrm{H}$ & & United States & 1984 & PITT 8417 \\
\hline Ala & 22 & 3733 & 9 & 5 & 1 & 5 & 5 & 3 & 5 & 2 & 2 & 4 & 5 & 3 & 4 & 2 & 4 & 2 & 2 & b & $2 \mathrm{~L}$ & & United States & 1984 & PITT 8430 \\
\hline Ala & 23 & 2793 & 5 & 5 & 1 & 5 & 5 & 3 & 5 & 2 & 2 & 4 & 5 & 3 & 4 & 2 & 4 & 2 & 2 & b & & & Scotland & 1984 & PEN $^{\dagger} 7866$ \\
\hline Ala & 24 & 2958 & 9 & 5 & 5 & 5 & 5 & 3 & 5 & 2 & 2 & 4 & 5 & 2 & 4 & 2 & 4 & 2 & 2 & b & $2 \mathrm{~L}$ & & United States & 1986 & SLCH $4691 \mathrm{~b}$ \\
\hline Ala & 26 & 1081 & 9 & 5 & 9 & 5 & 5 & 3 & 5 & 2 & 2 & 4 & 5 & 2 & 4 & 2 & 4 & 2 & 2 & b & $1 \mathrm{H}$ & G & United States & 1983 & SLCH 3765 \\
\hline Ala & 27 & 2932 & 9 & 5 & 9 & 5 & 5 & 3 & 5 & 2 & 2 & 2 & 5 & 2 & 4 & 2 & 4 & 2 & 2 & b & $1 \mathrm{~L}^{\ddagger}$ & & United States & 1985 & SLCH 4996A \\
\hline Ala & 28 & 250 & 9 & 5 & 1 & 5 & 5 & 3 & 5 & 2 & 1 & 4 & 5 & 2 & 4 & 2 & 4 & 2 & 2 & b & & & United States & $1980 \mathrm{~s}$ & \\
\hline $\mathrm{Ala}$ & 29 & 1079 & 9 & 5 & 1 & 5 & 5 & 3 & 5 & 2 & 1 & 0 & 5 & 2 & 4 & 2 & 4 & 2 & 2 & b & $2 \mathrm{~L}$ & $\mathrm{v}$ & United States & 1982 & SL.CH 3718 \\
\hline Ala & 30 & 1052 & 9 & 5 & 1 & 5 & 5 & 3 & 5 & 2 & 1 & 2 & 5 & 2 & 4 & 2 & 4 & 2 & 2 & b & $9 \mathrm{~L}$ & v & United States & 1980 & SLCH 628B \\
\hline Ala & 31 & 562 & 9 & 5 & 1 & 5 & 5 & 3 & 5 & 2 & 2 & 2 & 5 & 2 & 4 & 2 & 4 & 2 & 2 & b & & G & England & 1985 & RM6107 \\
\hline Ala & 32 & 1054 & 9 & 0 & 1 & 5 & 5 & 3 & 5 & 2 & 2 & 4 & 5 & 2 & 4 & 2 & 4 & 2 & 2 & b & $2 \mathrm{H}$ & & United States & 1980 & SLCH $85 \mathrm{Ib}$ \\
\hline Ala & 33 & 1868 & 9 & 4 & 1 & 5 & 5 & 3 & 5 & 2 & 2 & 4 & 5 & 2 & 4 & 2 & 4 & 2 & 2 & b & & & United States & $1980 \mathrm{~s}$ & LAW 31 \\
\hline Ala & 34 & 1080 & 9 & 0 & 1 & 5 & 5 & 3 & 6 & 2 & 2 & 4 & 5 & 2 & 4 & 2 & 4 & 2 & 2 & b & $1 \mathrm{~L}$ & G & United States & 1983 & SLCH 3752 \\
\hline Ala & 35 & 1358 & 8 & 0 & 1 & 5 & 5 & 3 & 6 & 2 & 2 & 4 & 5 & 2 & 4 & 2 & 4 & 2 & 2 & b & & & South Africa & $1980 \mathrm{~s}$ & $\mathrm{JOH}^{\S} 39746$ \\
\hline Alb & 36 & 1075 & 9 & 5 & 1 & 5 & 5 & 3 & 3 & 2 & 1 & 4 & 5 & 3 & 4 & 2 & 4 & 2 & 2 & b & $1 \mathrm{~L}$ & G & United States & $1980 \mathrm{~s}$ & SLCH 3438 \\
\hline Alc & 37 & 1200 & 9 & 5 & 4 & 5 & 5 & 3 & 5 & 2 & 2 & 4 & 5 & 2 & 4 & 2 & 4 & 2 & 2 & b & $2 \mathrm{~L}$ & & Canada & $1980 \mathrm{~s}$ & HAMEL QUE 167 \\
\hline Alc & 38 & 1083 & 9 & 5 & 4 & 5 & 5 & 3 & 6 & 2 & 2 & 4 & 5 & 2 & 4 & 2 & 4 & 2 & 2 & b & $\mathrm{IU}$ & & United States & 1984 & SLC \\
\hline Alc & 39 & 3093 & 5 & 5 & 4 & 5 & 5 & 3 & 6 & 2 & 2 & 4 & 5 & 2 & 4 & 2 & 4 & 2 & 2 & b & $3 \mathrm{~L}$ & s & Norway & $1970 \mathrm{~s}$ & KILIAN 10390 \\
\hline Alc & 40 & 1126 & 5 & 5 & 4 & 5 & 5 & 3 & 1 & 2 & 2 & 4 & 5 & 2 & 4 & 2 & 4 & 2 & 2 & b & $3 \mathrm{~L}$ & & Spain & $1980 \mathrm{~s}$ & R 1713 \\
\hline Alc & 41 & 2069 & 5 & 5 & 4 & 5 & 5 & 3 & 5 & 2 & 2 & 4 & 5 & 6 & 4 & 2 & 4 & 2 & 2 & b & & & France & $1980 \mathrm{~s}$ & DABERNAT 1762 \\
\hline Alc & 42 & 977 & 5 & 5 & 4 & 5 & 5 & 3 & 5 & 2 & 2 & 4 & 5 & 3 & 4 & 2 & 4 & 2 & 2 & b & $3 \mathrm{~L}$ & & Switzerland & 1985 & N 76 \\
\hline Alc & 43 & 945 & 5 & 5 & 4 & 5 & 5 & 7 & 5 & 2 & 2 & 4 & 5 & 3 & 4 & 2 & 4 & 2 & 2 & b & $11 \mathrm{~L}$ & & Switzerland & 1985 & N 108 \\
\hline Alc & 44 & 3084 & 9 & 5 & $\mathfrak{I}$ & 5 & 5 & 3 & 6 & 2 & 2 & 4 & 5 & 3 & 4 & 2 & 4 & 2 & 2 & b & IL & & United States & 1984 & PITT 8434 \\
\hline Alc & 45 & 1477 & 8 & 5 & 1 & 5 & 5 & 3 & 6 & 2 & 2 & 4 & 5 & 3 & 4 & 2 & 4 & 2 & 2 & b & IL & & The Gambia & 1982 & WALL 9 \\
\hline Alc & 46 & 2325 & 8 & 5 & 4 & 5 & 5 & 3 & 6 & 2 & 2 & 4 & 5 & 3 & 4 & 2 & 4 & 2 & 2 & b & & & Australia & $1980 \mathrm{~s}$ & RBH 24 \\
\hline Alc & 47 & 2976 & 7 & 5 & 4 & 5 & 5 & 3 & 3 & 2 & 2 & 4 & 5 & 3 & 4 & 2 & 4 & 2 & 2 & b & & & Scotland & 1986 & PEN $\dagger^{\dagger} 3943$ \\
\hline Alc & 48 & 1176 & 7 & 5 & 4 & 5 & 5 & 3 & 6 & 2 & 2 & 4 & 5 & 3 & 4 & 2 & 4 & 2 & 2 & b & $26 \mathrm{~L}$ & & Canada & $1980 \mathrm{~s}$ & HAMEL C $126 / 83$ \\
\hline Ald & 49 & 2957 & 9 & 5 & 1 & 5 & 5 & 7 & 3 & 2 & 2 & 4 & 5 & 2 & 4 & 2 & 4 & 2 & 2 & b & $1 \mathrm{H}$ & & United States & 1986 & SLCH $4630 C$ \\
\hline Ald & so & 701 & 8 & 5 & 1 & 5 & 5 & 7 & 3 & 2 & 2 & 4 & 5 & 2 & 4 & 2 & 4 & 2 & .2 & b & & $\mathrm{v}$ & Ghana & 1983 & RM7020 \\
\hline Ald & 51 & 2422 & 8 & 5 & 4 & 5 & 5 & 6 & 3 & 2 & 2 & 4 & 5 & 2 & 4 & 2 & 4 & 2 & 2 & b & & & Scotand & $1980 \mathrm{~s}$ & \\
\hline Ale & 52 & 812 & 8 & 5 & 1 & 5 & 1 & 6 & 3 & 2 & 2 & 4 & 5 & 3 & 4 & 2 & 4 & 2 & 2 & b & & & Ghana & $1980 \mathrm{~s}$ & RM7017 \\
\hline Alf & 53 & 2819 & 6 & 5 & 1 & 5 & 5 & 3 & 6 & 2 & 2 & 4 & 4 & 6 & 4 & 2 & 4 & 2 & 2 & $b$ & & $\mathrm{v}$ & Denmark & 1944 & ENGBAEK $\$ 41$ \\
\hline A2a & 54 & 3085 & 8 & 5 & 1 & 5 & 5 & 3 & 6 & 2 & 2 & 4 & 5 & 6 & 4 & 2 & 4 & 2 & 2 & b & IL & & Cor & 1984 & $\mathrm{HA}$ \\
\hline A2a & 55 & 1964 & 8 & 5 & 4 & 5 & 5 & 3 & 6 & 2 & 2 & 4 & 5 & 6 & 4 & 2 & 4 & 2 & 2 & b & & & Canada & $1980 \mathrm{~s}$ & LAW 127 \\
\hline A2a & 56 & 1491 & 8 & 5 & 4 & 5 & 5 & 3 & 5 & 2 & 2 & 4 & 5 & 6 & 4 & 2 & 4 & 2 & 2 & b & & & The Gan & 1983 & WALL 40 \\
\hline A2a & 57 & 1072 & 8 & 5 & 4 & 5 & 5 & 3 & 1 & 2 & 2 & 4 & 5 & 6 & 4 & 2 & 4 & 2 & 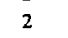 & b & $3 \mathrm{~L}$ & & United States & $1980 \mathrm{~s}$ & SLCH 3254 \\
\hline A2a & 58 & 1653 & 8 & 5 & 1 & 5 & 5 & 3 & 3 & 2 & 2 & 4 & $s$ & 6 & 4 & 2 & 4 & 2 & 2 & b & $16 \mathrm{~L}$ & & Malaysia & 1971 & SN0053 \\
\hline A2a & 12.8 & 1666 & 8 & 5 & 4 & 5 & 5 & 3 & 3 & 2 & 2 & 4 & 5 & 6 & 4 & 2 & 4 & 2 & 2 & b & $3 \mathrm{~L}$ & & United States & 1980 & SLCH 3010 \\
\hline A2a & 12.8 & 1064 & $B$ & 5 & 4 & 5 & 5 & 3 & 3 & 2 & 2 & 4 & 5 & 6 & 4 & 2 & 4 & 2 & 2 & b & IL & $\mathbf{s}$ & Thailand & 1979 & SLCH 1493A \\
\hline A2a & 59 & 2141 & 8 & 5 & 4 & 5 & 5 & 3 & 3 & 2 & 2 & 4 & 5 & 3 & 4 & 2 & 4 & 2 & 2 & b & $1 \mathrm{~L}$ & & Thailand & $1980 \mathrm{~s}$ & SLCH 8035 \\
\hline A2a & 60 & 1800 & 8 & 5 & 4 & 5 & 5 & 3 & 3 & 2 & 2 & 4 & 5 & 5 & 4 & 2 & 4 & 2 & 2 & $\mathrm{~b}$ & $3 \mathrm{~L}$ & $\mathbf{s}$ & Kenya & $1980 \mathrm{~s}$ & RM7419 \\
\hline A2a & 61 & 2821 & 8 & 5 & 4 & 5 & 5 & 8 & 3 & 2 & 2 & 4 & 5 & 6 & 4 & 2 & 4 & 2 & 2 & b & & & Denmark & 1983 & ARPI 20 \\
\hline$A 2 a$ & 62 & 1071 & 8 & 5 & 4 & 5 & 5 & 3 & 3 & 2 & 2 & 4 & 5 & 6 & 4 & 2 & 4 & 1 & 2 & b & $3 \mathrm{~L}$ & & United States & 1941 & SLCH 3207 \\
\hline $2 a$ & 63 & 3086 & 8 & 5 & 4 & 5 & 5 & 3 & 3 & 2 & 2 & 4 & 5 & 6 & 4 & 2 & 4 & 3 & 2 & $b$ & $3 \mathrm{~L}$ & & Norway & $1980 \mathrm{~s}$ & KILIAN 5687 \\
\hline
\end{tabular}


Table 2. (continued)

\begin{tabular}{|c|c|c|c|c|c|c|c|c|c|c|c|c|c|c|c|c|c|c|c|c|c|c|c|c|c|}
\hline \multirow[b]{2}{*}{ Group } & & & & & & & & & Ulele & indic & caled er & enzyme & te locus & & & & & & & & OMP & & & & \\
\hline & ET & no & CAK & NSP & PGI 1 & MAE & MDH & G6P & GOT & $\mathrm{ADK}$ & 6PG & PE2 & PEt & LAP & M & CAT & GLD & G3P F & FUM & type & type & pattern & source & isolation & Other designation \\
\hline A2a & 64 & 1078 & 8 & 5 & 4 & 5 & 5 & 3 & 3 & 2 & 2 & 4 & 1 & 6 & 4 & 2 & 4 & 2 & 2 & b & 3L & $s$ & United States & 1982 & SLCH 3715A \\
\hline A2a & 65 & 1888 & 8 & 5 & 4 & 5 & 5 & 3 & 3 & 2 & 2 & 4 & 7 & 6 & 4 & 2 & 4 & 2 & 2 & b & & & Canada & $1980 \mathrm{~s}$ & LAW S1 \\
\hline A2a & 66 & 1062 & 8 & 5 & 4 & 5 & 5 & 3 & 3 & 2 & 2 & 4 & 5 & 6 & 4 & 2 & 4 & 2 & 3 & b & $11 \mathrm{~L}$ & $s$ & United States & 1980 & SLCH 1445 \\
\hline A2a & 67 & 3734 & 8 & 5 & 4 & 5 & 5 & 3 & 3 & 2 & 2 & 4 & 5 & 6 & 4 & 2 & 11 & 2 & 2 & b & $3 \mathrm{~L}$ & & Norway & $1970 \mathrm{~s}$ & KILIAN 9703 \\
\hline A2a & 68 & 1313 & 8 & 5 & 4 & 5 & 5 & 3 & 3 & 2 & 2 & 4 & 5 & 6 & 4 & 2 & 1 & 2 & 2 & b & $3 \mathrm{~L}$ & & Norway & $1970 \mathrm{~s}$ & KILIAN 3754 \\
\hline$A 2 a$ & 69 & 1595 & 8 & 5 & 4 & 5 & 6 & 3 & 3 & 2 & 2 & 4 & 5 & 6 & 4 & 2 & 4 & 2 & 2 & b & & & Malaysia & 1975 & SN1352 \\
\hline A2a & 70 & 319 & 8 & 5 & 4 & 0 & 5 & 3 & 3 & 2 & 2 & 4 & 5 & 6 & 4 & 2 & 4 & 2 & 2 & b & & & Papua New Guinea & 1980 & SLCH 4294 \\
\hline$A 2 a$ & 71 & 558 & 8 & 5 & 4 & 5 & 5 & 3 & 3 & 2 & 2 & 2 & 5 & 6 & 4 & 2 & 4 & 2 & 2 & b & & G & England & 1983 & RM6094 \\
\hline A2a & 72 & 1751 & 8 & 5 & 4 & 5 & 5 & 3 & 3 & 3 & 2 & 2 & 5 & 6 & 4 & 2 & 4 & 2 & 2 & b & $3 \mathbf{L}$ & & Iceland & 1977 & B-3786 \\
\hline $\mathrm{A} 2 \mathrm{a}$ & 73 & 1245 & 8 & 5 & 4 & 5 & 5 & 3 & 3 & 2 & 2 & 5 & 5 & 6 & 4 & 2 & 4 & 2 & 2 & b & & & Canada & $1980 \mathrm{~s}$ & HAMEL MONTREAL \\
\hline A2a & 74 & 1301 & 8 & 5 & 2 & 5 & 5 & 3 & 3 & 2 & 2 & 5 & 5 & 6 & 4 & 2 & 4 & 2 & 2 & b & $2 \mathrm{~L}$ & & South Africa & $1980 \mathrm{~s}$ & CAPE TOWN 6559 \\
\hline A2a & 12.6 & 1058 & 6 & 5 & 4 & 5 & 5 & 3 & 3 & 2 & 2 & 4 & 5 & 6 & 4 & 2 & 4 & 2 & 2 & b & $14 \mathrm{~L}$ & $\mathbf{s}$ & United States & 1980 & SLCH 1256 \\
\hline A2a & 12,10 & 476 & $\mathbf{0}$ & 5 & 4 & 5 & 5 & 3 & 3 & 2 & 2 & 4 & 5 & 6 & 4 & 2 & 4 & 2 & 2 & b & $11 \mathrm{~L}$ & & Hawaii & $1980 \mathrm{~s}$ & WAGNER 741 \\
\hline A2a & 12.9 & 1373 & 9 & 5 & 4 & 5 & 5 & 3 & 3 & 2 & 2 & 4 & 5 & 6 & 4 & 2 & 4 & 2 & 2 & b & $16 \mathrm{~L}$ & $\mathrm{~s}$ & Japan & 1982 & KAWAKAMI 39 \\
\hline A2a & 75 & 3089 & 9 & 5 & 4 & 5 & 5 & 3 & $\mathbf{3}$ & 2 & 2 & 4 & 5 & 6 & 1 & 2 & 4 & 2 & 2 & b & $3 \mathrm{~L}$ & & Denrnark & $1980 \mathrm{~s}$ & KILIAN M1840 \\
\hline A2a & 12.11 & 2358 & 1 & 5 & 4 & 5 & 5 & 3 & 3 & 2 & 2 & 4 & 5 & 6 & 4 & 2 & 4 & 2 & 2 & b & $29 \mathrm{~L}$ & & Philippines & $1980 \mathrm{~s}$ & SLCH 8661 \\
\hline A2a & 76 & 1348 & 1 & 5 & 4 & 5 & 5 & 3 & 3 & 2 & 2 & 4 & 5 & 3 & 4 & 2 & 4 & 2 & 2 & b & & & South Africa & $1980 \mathrm{~s}$ & $\mathrm{JOH}^{8} 40422$ \\
\hline A2a & 12.7 & 3735 & 7 & 5 & 4 & 5 & 5 & 3 & 3 & 2 & 2 & 4 & 5 & 6 & 4 & 2 & 4 & 2 & 2 & b & $3 \mathrm{~L}$ & $s$ & United States & 1984 & PITT 8416 \\
\hline $\mathbf{A} 2 \mathrm{a}$ & 77 & 930 & 7 & 5 & 4 & 5 & 2 & 3 & 3 & 2 & 2 & 4 & 5 & 6 & 4 & 2 & 4 & 2 & 2 & b & $3 \mathrm{~L}$ & & Switzerland & 1983 & N 201 \\
\hline A2a & 78 & 1055 & 9 & 5 & 4 & 5 & 2 & 3 & 3 & 2 & 2 & 4 & 5 & 6 & 4 & 2 & 4 & 2 & 2 & $b$ & $16 \mathrm{~L}$ & $s$ & United States & $1970 \mathrm{~s}$ & SLCH 1059 \\
\hline A2a & 79 & 942 & 5 & 5 & 4 & 5 & 2 & 3 & 3 & 2 & 2 & 4 & 5 & 6 & 4 & 2 & 4 & 2 & 2 & b & $3 \mathrm{~L}$ & & Switzerland & 1985 & N 111 \\
\hline$A 2 a$ & 12.5 & 1060 & 5 & 5 & 4 & 5 & 5 & 3 & 3 & 2 & 2 & 4 & 5 & 6 & 4 & 2 & 4 & 2 & 2 & b & $3 \mathrm{~L}$ & $s$ & United States & $1950 \mathrm{~s}$ & SLCH 1333 \\
\hline A2a & 80 & 2833 & 5 & 5 & 4 & 5 & 5 & 3 & 3 & 2 & 2 & 4 & 5 & 3 & 4 & 2 & 4 & 2 & 2 & b & & & Denmark & 1983 & ARPI 68 \\
\hline $\mathrm{A} 2 \mathrm{a}$ & 81 & 1343 & 5 & 5 & 4 & 5 & 5 & 3 & 3 & 2 & 2 & 4 & 5 & 5 & 4 & 2 & 4 & 2 & 2 & b & 3L & & South Africa & $1980 \mathrm{~s}$ & JOH $^{8}$ LANCER \\
\hline A2a & 82 & 2852 & 5 & 5 & 4 & 5 & 5 & 3 & 3 & 2 & 2 & 4 & 5 & 2 & 4 & 2 & 4 & 2 & 2 & $b$ & & & Sweden & 1982 & NK 653/82 \\
\hline A2a & 83 & 2315 & 5 & 5 & 4 & 5 & 5 & 3 & 3 & 2 & 2 & 4 & 5 & 6 & 2 & 2 & 4 & 2 & 2 & b & & & Australia & $1980 \mathrm{~s}$ & RBH 14 \\
\hline $\mathrm{A} 2 \mathbf{a}$ & 84 & 3090 & 5 & 5 & 4 & 5 & 5 & 3 & 3 & 2 & 2 & 4 & 5 & 6 & 1 & 2 & 4 & 2 & 2 & b & & & Canada & 1984 & HAM 13 \\
\hline A2a & 85 & 3087 & 5 & 5 & 4 & 6 & 5 & 3 & 3 & 2 & 2 & 4 & 5 & 6 & 4 & 2 & 4 & 2 & 2 & b & 3L & & Canada & 1984 & HAM 16 \\
\hline A2a & 21.8 & 2575 & 8 & 5 & 4 & 5 & 5 & 6 & 3 & 2 & 2 & 4 & 5 & 6 & 4 & 2 & 4 & 2 & 2 & b & $1 \mathrm{~L}$ & & Finland & 1985 & IHI23222 \\
\hline A2a & 21.9 & 2820 & 9 & 5 & 4 & 5 & 5 & 6 & 3 & 2 & 2 & 4 & 5 & 6 & 4 & 2 & 4 & 2 & 2 & b & & $\mathbf{s}$ & Sweden & 1985 & RM7109 \\
\hline A2a & 21.5 & 2310 & 5 & 5 & 4 & 5 & 5 & 6 & 3 & 2 & 2 & 4 & 5 & 6 & 4 & 2 & 4 & 2 & 2 & b & & & Australia & 1984 & RBH 1 \\
\hline $\mathrm{A} 2 \mathrm{a}$ & 86 & 2955 & 5 & 5 & 4 & 5 & 5 & 8 & 3 & 2 & 2 & 4 & 5 & 6 & 4 & 2 & 4 & 2 & 2 & b & 3L & & United States & 1985 & SLCH $4585 B$ \\
\hline A2a & 87 & 2784 & 5 & 5 & 4 & 5 & 5 & 1 & 3 & 2 & 2 & 4 & 5 & 6 & 4 & 2 & 4 & 2 & 2 & b & & & Finland & 1985 & IHI23368 \\
\hline A2a & 88 & 2546 & 5 & 5 & 4 & 5 & 5 & 7 & 3 & 2 & 2 & 4 & 5 & 6 & 4 & 2 & 4 & 2 & 2 & b & & & Finland & 1985 & IHI23255 \\
\hline A2a & 89 & 2077 & 9 & 5 & 4 & 5 & 5 & 7 & 3 & 2 & 2 & 4 & 5 & 6 & 4 & 2 & 4 & 2 & 2 & b & & & France & 1980s & DABERNAT 1775 \\
\hline A2 $\mathbf{a}$ & 12.0 & 1694 & 0 & 5 & 4 & 5 & 5 & 3 & 3 & 2 & 2 & 4 & 5 & 6 & 4 & 2 & 4 & 2 & 2 & b & $3 \mathrm{~L}$ & & New Zealand & 1985 & AS850619 \\
\hline A2a & 90 & 2237 & 0 & 0 & 4 & 5 & 5 & 3 & 3 & 2 & 2 & 4 & 5 & 6 & 4 & 2 & 4 & 2 & 2 & b & & & Sweden & 1982 & NK 952 \\
\hline$A 2 a$ & 91 & 1840 & 7 & 0 & 4 & 5 & 5 & 3 & 3 & 2 & 2 & 4 & 5 & 6 & 4 & 2 & 4 & 2 & 2 & b & 3L & & Canada & $1980 \mathrm{~s}$ & LAW 3 \\
\hline $\mathrm{A} 2 \mathrm{a}$ & 92 & 1804 & 8 & 0 & 4 & 5 & 5 & 3 & 3 & 2 & 2 & 4 & 5 & 6 & 4 & 2 & 4 & 2 & 2 & b & & $s$ & Unired States & 1983 & RM1005 \\
\hline A2a & 93 & 2979 & 5 & 0 & 4 & 5 & 5 & 3 & 3 & 2 & 2 & 4 & 5 & 6 & 4 & 2 & 4 & 2 & 2 & b & & & Scotland & 1983 & $\mathrm{PEN}^{\dagger} 62782$ \\
\hline A2a & 94 & 2714 & 9 & 4 & 4 & 5 & 5 & 3 & 3 & 2 & 2 & 4 & 5 & 6 & 4 & 2 & 4 & 2 & 2 & b & & & United States & $1980 \mathrm{~s}$ & СНОР" 19 \\
\hline $\mathrm{A} 2 \mathrm{a}$ & 95 & 2708 & 8 & 4 & 4 & $s$ & 5 & 3 & 3 & 2 & 2 & 4 & 5 & 6 & 4 & 2 & 4 & 2 & 2 & b & & & United States & 1986 & CHOP" 13 \\
\hline A2a & 96 & 2824 & 5 & 4 & 4 & 5 & 5 & 3 & 3 & 2 & 2 & 4 & 5 & 6 & 4 & 2 & 4 & 2 & 2 & b & & & Greenland & 1983 & ARPI $89-83$ \\
\hline$A 2 a$ & 97 & 2609 & 7 & 4 & 4 & 5 & 5 & 3 & 3 & 2 & 2 & 4 & 5 & 6 & 4 & 2 & 4 & 2 & 2 & b & & & Canada & $1980 \mathrm{~s}$ & HAMEL PEII 26 \\
\hline A2a & 98 & 3088 & 7 & 7 & 4 & 5 & 5 & 3 & 3 & 2 & 2 & 4 & 5 & 6 & 4 & 2 & 4 & 2 & 2 & b & & & United States & I980s & YOGEV 111 \\
\hline$A 2 a$ & 99 & 1625 & 8 & 7 & 4 & 5 & 5 & 3 & 3 & 2 & 2 & 4 & 5 & 6 & 4 & 2 & 4 & 2 & 2 & b & $3 \mathrm{~L}$ & & Malaysia & 1975 & SN1204 \\
\hline $\mathrm{A} 2 \mathrm{a}$ & 100 & 1673 & 7 & 5 & 4 & 5 & 5 & 3 & 3 & 4 & 2 & 4 & 5 & 6 & 4 & 2 & 4 & 2 & 2 & b & $3 \mathrm{~L}$ & & Canada & 1980 & SLCH $1687 \mathrm{~F}$ \\
\hline A2a & 101 & 1067 & 7 & 5 & 4 & 5 & 5 & 3 & 3 & 2 & 3 & 4 & 5 & 6 & 4 & 2 & 4 & 2 & 2 & b 1 & 14.1L & $s$ & United States & 1980 & SLCH 1993 \\
\hline $\mathrm{A} 2 \mathrm{a}$ & 102 & 1077 & 7 & 5 & 4 & 5 & 5 & 3 & 3 & 2 & 4 & 4 & 5 & 6 & 4 & 2 & 4 & 2 & 2 & b & $22 \mathrm{~L}$ & $\mathrm{~s}$ & United States & 1980 & SLCH 3516 \\
\hline $\mathrm{A} 2 \mathrm{a}$ & 103 & 1057 & 7 & 5 & 4 & 5 & 5 & 3 & 3 & 2 & 2 & 4 & 5 & 6 & 6 & 2 & 4 & 2 & 2 & b & $5.1 \mathrm{~L}$ & & Alaska & 1980 & SLCH 1211 \\
\hline $\mathrm{A} 2 \mathrm{a}$ & 104 & 995 & 7 & 5 & 4 & 5 & 5 & 3 & 3 & 2 & 2 & 4 & 5 & 6 & 2 & 2 & 4 & 2 & 2 & b & $3 \mathrm{~L}$ & & Switzerland & 1986 & N 58 \\
\hline $\mathrm{A}_{2} \mathrm{a}$ & 105 & 1036 & 8 & 5 & 4 & 5 & 5 & 3 & 3 & 2 & 2 & 4 & 5 & 6 & 6 & 2 & 4 & 2 & 2 & b & & & Switzerland & 1985 & N 16 \\
\hline A2a & 106 & 3091 & 8 & 5 & 4 & 5 & 1 & 3 & 3 & 2 & 4 & 4 & 5 & 6 & 4 & 2 & 4 & 2 & 2 & $\mathrm{~b}$ & $13 \mathrm{~L}$ & & Alaska & 1985 & JW 19 \\
\hline $\mathrm{A} 2 \mathrm{a}$ & 107 & 3092 & 8 & 5 & 4 & 5 & 5 & 3 & 3 & 2 & 1 & 4 & 5 & 6 & 4 & 2 & 4 & 2 & 2 & b & & & Canada & 1969 & TOR 31 \\
\hline A2a & 108 & 933 & 5 & 5 & 4 & 5 & 5 & 3 & 3 & 2 & 1 & 4 & 5 & 6 & 4 & 2 & 4 & 2 & 2 & b & 3L & & Switzerland & 1983 & N 204 \\
\hline$A 2 a$ & 109 & 1305 & 8 & 5 & 2 & 5 & 5 & 3 & 3 & 2 & 1 & 4 & 5 & 6 & 4 & 2 & 4 & 2 & 2 & b & $2 \mathrm{~L}$ & & South Africa & $1980 \mathrm{~s}$ & CAPE TOWN 9723 \\
\hline A2a & 110 & 3736 & 5 & 5 & 4 & 5 & 5 & 3 & 3 & 2 & 2 & 4 & 5 & 6 & 4 & 2 & 12 & 2 & 2 & b & $1 \mathrm{~L}$ & & Denmark & & KILIAN 114 \\
\hline A2a & 111 & 1255 & 5 & 5 & 4 & 5 & 5 & 3 & 3 & 2 & 2 & 4 & 5 & 6 & 4 & 2 & 12 & 3 & 2 & b & & & Canada & $1980 \mathrm{~s}$ & \\
\hline $\mathrm{A} 2 \mathrm{~b}$ & 112 & 1937. & 7 & 5 & 5 & 5 & 5 & 3 & 3 & 2 & 2 & 4 & 5 & 6 & 4 & 2 & 4 & 2 & 2 & b & & & Canada & $1980 \mathrm{~s}$ & LAW 100 \\
\hline $\mathrm{A} 2 \mathrm{~b}$ & 113 & 1244 & 8 & 5 & 5 & 5 & 5 & 3 & 3 & 2 & 2 & 4 & 5 & 6 & 4 & 3 & 4 & 2 & 2 & b & & & Canada & $1980 \mathrm{~s}$ & CHILETTE \\
\hline $\mathrm{A} 2 \mathrm{~b}$ & 114 & 3737 & 6 & 5 & 1 & 5 & 5 & 3 & 3 & 2 & 2 & 4 & 5 & 6 & 4 & 3 & 4 & 2 & 2 & b & $5 \mathrm{~L}$ & & Alaska & $1980 \mathrm{~s}$ & JW 81 \\
\hline $\mathrm{A} 2 \mathrm{c}$ & 115 & 1314 & 8 & 5 & 4 & 5 & 5 & 3 & 3 & 2 & 2 & 4 & 4 & 5 & 4 & 2 & 4 & 2 & 2 & b & $2 L$ & & South Africa & $1980 \mathrm{~s}$ & DURBAN 63 \\
\hline A2d & 116 & 188 & 8 & 5 & 4 & 5 & 3 & 6 & 3 & 2 & 2 & 4 & 5 & 6 & 4 & 2 & 4 & 2 & 2 & $\mathrm{~b}$ & 16L & & Dominican Republic & $1980 \mathrm{~s}$ & CEF 79 \\
\hline A2d & 117 & 212 & 5 & 5 & 4 & 5 & 3 & 2 & 3 & 2 & 2 & 4 & 5 & 6 & 4 & 2 & 4 & 2 & 2 & b & & & Dominican Republic & $1980 \mathrm{~s}$ & SULB 37 \\
\hline A2d & 118 & 1059 & 7 & 5 & 4 & 5 & 3 & 3 & 3 & 2 & 2 & 4 & 5 & 6 & 4 & 2 & 4 & 2 & 1 & b & $5 \mathrm{~L}$ & $s$ & United States & $1980 \mathrm{~s}$ & SLCH 1287 \\
\hline$A 2 e$ & 21.6 & 1053 & 6 & 5 & 4 & 5 & 5 & 6 & 3 & 2 & 2 & 4 & 5 & 6 & 4 & 2 & 4 & 2 & 2 & b & $13 \mathrm{~L}$ & & United States & 1979 & SLCH 808A \\
\hline A2e & 119 & 1068 & 6 & 5 & 4 & 5 & 5 & 6 & 3 & 2 & 3 & 4 & 5 & 6 & 4 & 2 & 4 & 2 & 2 & b & $11 \mathrm{~L}$ & & United States & $1980 \mathrm{~s}$ & SLCH 3012 \\
\hline aze & 120 & 3095 & 6 & 5 & 4 & 5 & 5 & 6 & 3 & 2 & 2 & 4 & 5 & 6 & 6 & 2 & 4 & 2 & 2 & b & $13 \mathrm{~L}$ & & Alaska & $1980 \mathrm{~s}$ & JW 80 \\
\hline
\end{tabular}


Table 2. (continued)

\begin{tabular}{|c|c|c|c|c|c|c|c|c|c|c|c|c|c|c|c|c|c|c|c|c|c|c|c|c|c|}
\hline \multirow{2}{*}{\multicolumn{2}{|c|}{ Group ET }} & \multirow{2}{*}{$\begin{array}{c}\text { MHAEM } \\
\text { no.* }\end{array}$} & \multicolumn{17}{|c|}{ Allele at indicated enzyme locus } & \multirow{2}{*}{\multicolumn{2}{|c|}{$\begin{array}{l}\text { Sero- OMP } \\
\text { type type }\end{array}$}} & \multirow{2}{*}{$\begin{array}{c}\text { RFLP } \\
\text { pattern }\end{array}$} & \multirow{2}{*}{$\begin{array}{c}\text { Geographic } \\
\text { source }\end{array}$} & & \\
\hline & & & CAK & NSP & PGI & MAE & MDH & G6P & GOT & ADK & $6 \mathrm{PG}$ & PE2 & PE1 & LAP & PGM & CAT & T GLD & G3P & FUM & & & & & isolation & Other designation \\
\hline A2e & 121 & 472 & 6 & 5 & 4 & 5 & 5 & 6 & 3 & 2 & 2 & 4 & 4 & 6 & 4 & 2 & 4 & 2 & 2 & b & $13 \mathrm{~L}$ & & Hawaii & 1980 & 2862 \\
\hline $\mathrm{A} 2 \mathrm{e}$ & 122 & 1778 & 6 & 5 & 5 & 5 & 5 & 6 & 3 & 2 & 2 & 4 & 5 & 6 & 4 & 2 & 4 & 2 & 2 & b & & $\mathbf{s}$ & United States & 1984 & RM8012 \\
\hline$A 2 e$ & 123 & 1092 & 6 & 5 & 4 & 3 & 5 & 6 & 3 & 2 & 2 & 4 & 5 & 6 & 4 & 2 & 4 & 2 & 2 & b & & & United States & $1980 \mathrm{~s}$ & \\
\hline A2e & 124 & 3096 & 6 & 0 & 4 & 5 & 5 & 6 & 3 & 2 & 1 & 4 & 5 & 6 & 4 & 2 & 4 & 2 & 2 & b & $13 \mathrm{~L}$ & & Alaska & $1980 \mathrm{~s}$ & JW 67 \\
\hline$A 2 e$ & 125 & 2716 & 6 & 5 & 4 & 5 & 5 & 6 & 3 & 2 & 2 & 4 & 7 & 3 & 4 & 2 & 4 & 2 & 2 & $\mathrm{~b}$ & & & United States & $1980 \mathrm{~s}$ & CHOP" 21 \\
\hline A2e & 126 & 2809 & 6 & 5 & 4 & 5 & 5 & 7 & 3 & 2 & 2 & 5 & 5 & 6 & 4 & 2 & 4 & 2 & 2 & $b$ & & & Denmark & 1944 & ENGBAEK T28 \\
\hline A2f & 127 & 2804 & 6 & 5 & 4 & 3 & 2 & 3 & 3 & 2 & 2 & 4 & 5 & 6 & 4 & 2 & 4 & 2 & 2 & b & & & Denmark & 1943 & ENGBAEK K7 \\
\hline A3 & 128 & 484 & 6 & 5 & 1 & 3 & 5 & 3 & 3 & 2 & 2 & 4 & 5 & 3 & 4 & 12 & 4 & 2 & 2 & b & & & Hawaii & $1980 \mathrm{~s}$ & WAGNER 6546 \\
\hline A4 & 129 & 1670 & 6 & 5 & 1 & 3 & 5 & 7 & 1 & 2 & 2 & 4 & 8 & 8 & 4 & 2 & 4 & 2 & 2 & b & & & Canada & $1980 \mathrm{~s}$ & SLCH $1687 \mathrm{C}$ \\
\hline A5 & 130 & 1300 & 4 & 7 & 1 & 5 & 6 & 6 & 3 & 2 & 1 & 4 & 5 & 2 & 4 & 2 & 4 & 2 & 2 & b & IL & & South Africa & $1980 \mathrm{~s}$ & CAPE TOWN 5274 \\
\hline B 1a & 131 & 3097 & 6 & 5 & 4 & 5 & 3 & 6 & 3 & 2 & 2 & 4 & 5 & 3 & 6 & 2 & 4 & 2 & 2 & b & & & United States & $1980 \mathrm{~s}$ & RGH 5531 \\
\hline Bla & 132 & 1629 & 6 & 5 & 4 & 5 & 3 & 6 & 3 & 1 & 2 & 4 & 5 & 3 & 6 & 2 & 4 & 2 & 2 & b & & & Malaysia & 1973 & SN0573 \\
\hline Blb & 25.6 & 1063 & 6 & 5 & 5 & 3 & 3 & 6 & 3 & 2 & 2 & 4 & 5 & 3 & 6 & 2 & 4 & 2 & 2 & b & $6 \mathrm{U}$ & $\mathbf{s}$ & United States & 1981 & SLCH 1481a \\
\hline Blb & 133 & 798 & 6 & 5 & 5 & 3 & 3 & 6 & 3 & 2 & 3 & 4 & 5 & 3 & 6 & 2 & 4 & 2 & 2 & b & & $\mathbf{s}$ & United States & 1985 & RM8069 \\
\hline Blb & 134 & 1973 & 6 & 5 & 5 & 3 & 3 & 6 & 6 & 2 & 2 & 4 & 5 & 3 & 6 & 2 & 4 & 2 & 2 & b & & & Canada & $1980 \mathrm{~s}$ & LAW 136 \\
\hline $\mathbf{B} 1 \mathbf{b}$ & 135 & 2163 & 6 & 5 & 5 & 3 & 3 & 6 & 3 & 2 & 2 & 3 & 5 & 3 & 6 & 2 & 4 & 2 & 2 & b & $6 U$ & & Papua New Guinea & 1980 & SLCH 8362a \\
\hline B1b & 136 & 2033 & 6 & 5 & 5 & 3 & 3 & 6 & 3 & 2 & 2 & 4 & 5 & 6 & 6 & 2 & 4 & 2 & 2 & b & & & Hawaij & 1986 & WAGNER 2954 \\
\hline B1b & 137 & 2135 & 6 & 5 & 5 & 3 & 3 & 6 & 3 & 2 & 2 & 4 & 5 & 6 & 4 & 2 & 4 & 2 & 2 & b & & & France & & DABERNAT 2280 \\
\hline B1b & 138 & 881 & 6 & 5 & 5 & 3 & 3 & 6 & 3 & 2 & 2 & 4 & 5 & 3 & 4 & 2 & 4 & 2 & 2 & b & $6 \mathrm{U}$ & $\mathbf{s}$ & The Netherlands & 1977 & 770177 \\
\hline B1b & 139 & 2139 & 6 & 5 & 5 & 3 & 3 & 6 & 3 & 2 & 2 & 4 & 5 & 5 & 4 & 2 & 4 & 2 & 2 & b & $6 \mathrm{U}$ & & Kenya & 1980 & SLCH 8009 \\
\hline B1b & 140 & 1795 & 6 & 5 & 5 & 3 & 3 & 7 & 3 & 2 & 2 & 4 & 5 & 5 & 4 & 2 & 4 & 2 & 2 & $\mathrm{~b}$ & & $\mathbf{s}$ & Kenya & $1980 \mathrm{~s}$ & RM7414 \\
\hline B1b & 141 & 1481 & 6 & 5 & 5 & 3 & 3 & 6 & 3 & 2 & 2 & 4 & 5 & 5 & 6 & 2 & 4 & 2 & 2 & $\mathbf{b}$ & $6 \mathrm{U}$ & & The Gambia & 1982 & WALL 13 \\
\hline B1b & 142 & 1480 & 6 & 5 & 5 & 3 & 3 & 6 & 3 & 2 & 2 & 4 & 5 & 5 & 6 & 2 & 4 & 2 & 1 & b & & & Hawaii & $1980 \mathrm{~s}$ & WAGNER 6618 \\
\hline B1b & 143 & 1056 & 6 & 5 & 5 & 3 & 3 & 6 & 3 & 2 & 2 & 4 & 5 & 3 & 6 & 2 & 4 & 2 & 1 & $\mathbf{b}$ & $23 \mathrm{U}$ & $\mathbf{s}$ & United States & 1985 & SLCH 1209 \\
\hline B1b & 144 & 1493 & 6 & 5 & 5 & 3 & 3 & 3 & 3 & 2 & 2 & 4 & 5 & 5 & 6 & 2 & 4 & 2 & 2 & b & & & The Gambia & & WALL 42 \\
\hline B1b & 145 & 1066 & 6 & 5 & 5 & 3 & 3 & 6 & 3 & 2 & 2 & 4 & 5 & 2 & 6 & 2 & 4 & 2 & 2 & b & $24 \mathrm{U}$ & $\mathbf{s}$ & United States & 1982 & SLCH 1971 \\
\hline B1b & 146 & 3098 & 6 & 5 & 5 & 3 & 3 & 7 & 3 & 2 & 2 & 4 & 5 & 2 & 6 & 2 & 4 & 2 & 2 & b & & & United States & 1984 & PITT 8447 \\
\hline Blb & 147 & 171 & 6 & 7 & 5 & 3 & 3 & 6 & 3 & 2 & 2 & 4 & 5 & 2 & 6 & 2 & 4 & 2 & 2 & $\mathrm{~b}$ & & & Dominican Republic & 1980 & CEF 8 \\
\hline Blb & 148 & 215 & 6 & 7 & 5 & 3 & 3 & 6 & 3 & 2 & 2 & 4 & 5 & 3 & 6 & 2 & 4 & 2 & 2 & $b$ & & & Dominican Republic & $1980 \mathrm{~s}$ & SULB 83 \\
\hline Blb & 149 & 225 & 6 & 7 & 5 & 3 & 3 & 3 & 3 & 2 & 2 & 4 & 5 & 2 & 6 & 2 & 4 & 2 & 2 & $\mathrm{~b}$ & $6 \mathrm{U}$ & & Dominican Republic & $1980 \mathrm{~s}$ & SULB 85 \\
\hline Bib & 150 & 874 & 6 & 5 & 5 & 3 & 3 & 6 & 3 & 2 & 2 & 4 & 7 & 5 & 4 & 2 & 4 & 2 & 2 & b & $6 \mathrm{U}$ & & South Africa & 1984 & 840062 \\
\hline B1b & 151 & 912 & 6 & 5 & 5 & 3 & 3 & 6 & 3 & 2 & 2 & 4 & 7 & 6 & 4 & 2 & 4 & 2 & 2 & b & & $\mathbf{s}$ & Switzerland & 1985 & N 207 \\
\hline B1b & 152 & 1334 & 6 & 5 & 5 & 3 & 3 & 6 & 3 & 2 & 2 & 4 & 7 & 5 & 6 & 2 & 4 & 2 & 2 & b & $6 \mathrm{U}$ & $\mathbf{s}$ & South Africa & 1980 & 12681 \\
\hline Blb & 153 & 1347 & 6 & 0 & 5 & 3 & 3 & 6 & 3 & 2 & 2 & 4 & 7 & 5 & 6 & 2 & 4 & 2 & 2 & $b$ & $6 \mathrm{U}$ & & South Africa & $1980 \mathrm{~s}$ & $\mathrm{JOH}^{8} 128011$ \\
\hline Blc & 154 & 206 & 5 & 5 & 5 & 3 & 3 & 3 & 3 & 2 & 2 & 4 & 5 & 6 & 6 & 2 & 4 & 2 & 2 & b & & & Dominican Republic & $1980 \mathrm{~s}$ & SULB 31 \\
\hline Bld & 155 & 3100 & 6 & 5 & 5 & 3 & 3 & 1 & 3 & 2 & 2 & 4 & 5 & 12 & 6 & 2 & 4 & 1 & 2 & b & $\mathbf{I U}$ & & Alaska & $1980 \mathrm{~s}$ & JW 65 \\
\hline Ble & 156 & 3099 & 6 & 5 & 5 & 3 & 3 & 3 & 3 & 2 & 2 & 3 & 5 & 3 & 4 & 2 & 4 & 2 & 2 & b & & & South Korea & $1980 \mathrm{~s}$ & D14 \\
\hline Blf & 157 & 571 & 6 & 5 & 5 & 3 & 5 & 6 & 3 & 2 & 2 & 4 & 5 & 3 & 6 & 2 & 4 & 2 & 2 & d & & D & England & 1963 & RM6137 \\
\hline BIf & 158 & 518 & 6 & 5 & 5 & 3 & 5 & 6 & 3 & 2 & 2 & 4 & 5 & 3 & 6 & 2 & 4 & 3 & 2 & d & & $\mathrm{D}$ & United States & & RMI 168 \\
\hline Blf & 159 & 584 & 6 & 5 & 5 & 3 & 5 & 6 & 1 & 2 & 2 & 4 & 5 & 3 & 6 & 2 & 4 & 2 & 2 & d & & D & England & 1985 & RM6150 \\
\hline B1f & 160 & 787 & 6 & 5 & 5 & 5 & 5 & 6 & 1 & 2 & 2 & 4 & 5 & 3 & 6 & 2 & 4 & 2 & 2 & d & & D & United States & 1984 & RM8039 \\
\hline Blf & 161 & 706 & 6 & 5 & 5 & 3 & 5 & 6 & 1 & 2 & 2 & 4 & 5 & 3 & 6 & 2 & 11 & 2 & 2 & d & & D & Papua New Guinea & $1980 \mathrm{~s}$ & RM7033 \\
\hline B1g & 162 & 747 & 6 & 5 & 5 & 3 & 5 & 6 & 3 & 2 & 2 & 2 & 5 & 3 & 6 & 5 & 4 & 2 & 2 & d & & D & Malaysia & 1972 & RM7271 \\
\hline$B \lg$ & 163 & 1810 & 6 & 5 & 5 & 3 & 5 & 6 & 3 & 2 & 2 & 2 & 5 & 5 & 4 & 2 & 4 & 2 & 2 & d & & D & Kenya & 1986 & RM7429 \\
\hline $\mathbf{B} \mathbf{a} \mathbf{a}$ & 164 & 506 & 6 & 5 & 6 & 5 & 5 & 6 & 3 & 2 & 2 & 4 & 5 & 2 & 1 & 2 & 4 & 2 & 3 & a & $1 \mathrm{U}$ & $\mathbf{T}$ & United States & 1981 & RM1042 \\
\hline $\mathbf{B 2 b}$ & 165 & 704 & 6 & 5 & 5 & 3 & 5 & 6 & 3 & 2 & 2 & 4 & 5 & 2 & 1 & 2 & 4 & 2 & 2 & a & 10 & $\mathbf{T}$ & Papua New Guinea & $1980 \mathrm{~s}$ & RM7031 \\
\hline $\mathbf{B} 2 \mathbf{b}$ & 166 & 705 & 6 & 5 & 5 & 3 & 5 & 6 & 3 & 2 & 2 & 4 & 5 & 2 & 1 & 2 & 4 & 3 & 2 & a & $1 \mathrm{U}$ & $\mathbf{T}$ & Papua New Guinea & $1980 \mathrm{~s}$ & RM7032 \\
\hline $\mathbf{B} 2 \mathbf{b}$ & 167 & 728 & 6 & 5 & 5 & $\mathfrak{3}$ & 5 & 6 & 3 & 2 & 2 & 4 & 5 & 3 & 1 & 2 & 4 & 2 & 3 & $\mathbf{a}$ & 10 & $\mathbf{T}$ & Malaysia & 1973 & RM7191 \\
\hline $\mathbf{B} 2 \mathbf{b}$ & 168 & 735 & 6 & 5 & 5 & 3 & 5 & 6 & 3 & 2 & 2 & 4 & 6 & 3 & 1 & 2 & 4 & 2 & 3 & a & 10 & $\mathbf{T}$ & Malaysia & 1974 & RM7198 \\
\hline $\mathbf{B} 2 \mathrm{c}$ & 169 & 727 & 6 & 5 & 5 & 3 & $s$ & 6 & 1 & 2 & 2 & 2 & 5 & 6 & 1 & 2 & 4 & 2 & 3 & a & $1 U$ & $\mathbf{T}$ & Malaysia & 1973 & RM7190 \\
\hline B2d & 170 & 1802 & 6 & 5 & 5 & 3 & 5 & 6 & 6 & 2 & 2 & 4 & 5 & 5 & 3 & 2 & 4 & 2 & 3 & a & $1 \mathrm{U}$ & $\mathbf{N}$ & Kenya & 1986 & RM7421 \\
\hline B3 & 171 & 1485 & 6 & 5 & 11 & 2 & 3 & 6 & 5 & 2 & 2 & 4 & 5 & 3 & 4 & 2 & 4 & 2 & 2 & b & & & The Gambia & 1983 & WALL 18 \\
\hline B4 & 172 & 1370 & 6 & 5 & 2 & 3 & 2 & 6 & 1 & 2 & 2 & 4 & 5 & 2 & 4 & 2 & 4 & 2 & 2 & $b$ & & & Japan & 1985 & KAWAKAMI 10 \\
\hline B4 & 173 & 742 & 6 & 5 & 4 & 3 & 2 & 6 & 1 & 2 & 2 & 4 & 5 & 5 & 4 & 2 & 4 & 2 & 3 & $\mathbf{a}$ & 5L & $\mathbf{N}$ & The Gambia & $1980 \mathrm{~s}$ & RM7205 \\
\hline B4 & 174 & 1797 & 6 & 5 & 4 & 3 & 2 & 6 & 1 & 2 & 2 & 4 & 5 & 3 & 4 & 2 & 4 & 2 & 3 & $\mathbf{a}$ & $\mathbf{S L}$ & $\mathbf{N}$ & Kenya & 1986 & RM7416 \\
\hline B5 & 175 & 1788 & 6 & 5 & 1 & 3 & 3 & 8 & 6 & 2 & 2 & 5 & 4 & 3 & 6 & 2 & 4 & 2 & 2 & b & & & Iceland & 1986 & B-1014 \\
\hline Cl & 176 & 993 & 4 & 5 & 4 & 2 & 3 & 8 & 5 & 2 & 3 & 5 & 5 & 6 & 4 & 3 & 4 & 1 & 2 & b & & & Switzerland & 1985 & $\mathrm{~N} 60$ \\
\hline Dla & 177 & 517 & 6 & 5 & 10 & 5 & 1 & 6 & 5 & 2 & 2 & 4 & 1 & 3 & 6 & 2 & 4 & 3 & 2 & c & & $\mathrm{Cl}$ & United States & & RM1167 \\
\hline D1a & 178 & 746 & 6 & 51 & 10 & 5 & 1 & 6 & 5 & 2 & 2 & 4 & 1 & 3 & 6 & 2 & 4 & 2 & 3 & c & & $\mathrm{Cl}$ & Malaysia & 1975 & RM7270 \\
\hline
\end{tabular}


Table 2. (continued)

\begin{tabular}{|c|c|c|c|c|c|c|c|c|c|c|c|c|c|c|c|c|c|c|c|c|c|c|c|c|c|}
\hline & & & & & & & & & Allele a & t indic & cated e & enzyme & e locus & & & & & & & & & & & & \\
\hline Group & & no. ${ }^{*}$ & CAK & NSP & PGI & MAE & MDH & I G6P & GOT & ADK & $6 \mathrm{PG}$ & PE2 & $\mathbf{P E 1}$ & LAP $P$ & PGM & CAT & GLD & G3P 1 & FUM & type & type $p$ & pattern & source & isolation & Other designation \\
\hline Dlb & 179 & 520 & 6 & 0 & 10 & 5 & 1 & 6 & 5 & 2 & 2 & 4 & 1 & 2 & 6 & 2 & 4 & 2 & 2 & c & & & Unknown & 1968 & RM1271 \\
\hline Dlb & 180 & 743 & 6 & 0 & 10 & 5 & 1 & 6 & 5 & 2 & 2 & 4 & 3 & 5 & 6 & 2 & 4 & 2 & 2 & c & & Cl & Malaysia & 1973 & RM7267 \\
\hline DIb & 181 & 569 & 6 & 0 & 10 & 5 & 5 & 6 & 5 & 2 & 2 & 4 & 5 & 2 & 6 & 2 & 4 & 2 & 2 & c & & Cl & England & 1981 & RM6135 \\
\hline D2 & 182 & 565 & 6 & 5 & 10 & 5 & 5 & 6 & 5 & 2 & 2 & 4 & 3 & 6 & 6 & 2 & 4 & 2 & 1 & c & & C1 & Unknown & 1970 & RM6129 \\
\hline D2 & 183 & 566 & 6 & 5 & 10 & 5 & 5 & 5 & 5 & 2 & 2 & 4 & 3 & 6 & 4 & 2 & 4 & 2 & 2 & c & & C1 & England & 1964 & RM6132 \\
\hline D3 & 184 & 785 & 6 & 0 & 13 & 5 & 1 & 3 & 5 & 2 & 2 & 4 & 5 & 6 & 6 & 2 & 4 & 3 & 2 & c & & Cl & United States & 1983 & RM8032 \\
\hline D4 & 185 & 568 & 6 & 5 & 7 & 5 & 1 & 7 & 3 & 2 & 2 & 4 & 4 & 3 & 6 & 2 & 4 & 3 & 2 & c & & $\mathrm{C} 2$ & England & 1975 & RM6134 \\
\hline DS & 186 & 1803 & 6 & 5 & 7 & 5 & 1 & 7 & 5 & 2 & 2 & 3 & 4 & 2 & 4 & 2 & 4 & 2 & 2 & c & & C2 & Kenya & 1986 & RM7422 \\
\hline E1 & 187 & 1805 & 5 & 5 & 1 & 5 & 5 & 6 & 1 & 2 & 2 & 5 & 7 & 1 & 3 & 2 & 4 & 2 & 1 & c & & & Kenya & 1986 & RM7424 \\
\hline F1 & 188 & 602 & 5 & 5 & 9 & 2 & 5 & 6 & 3 & 2 & 2 & 4 & 3 & 3 & 7 & 2 & 5 & 2 & 2 & e & & E & England & 1964 & RM6168 \\
\hline FI & 189 & 603 & 5 & 5 & 9 & 2 & 5 & 6 & 3 & 2 & 2 & 4 & 3 & 3 & 7 & 2 & 1 & 2 & 2 & e & & $\mathbf{E}$ & England & 1964 & RM6169 \\
\hline F1 & 190 & 619 & 5 & 5 & 9 & 2 & 5 & 6 & 3 & 2 & 2 & 4 & 5 & 3 & 7 & 2 & 1 & 2 & 2 & e & & $\mathbf{E}$ & England & 1965 & RM6185 \\
\hline $\mathrm{F} 2 \mathrm{a}$ & 191 & 492 & 9 & 5 & 9 & 2 & 5 & 1 & 6 & 2 & 4 & 2 & 3 & 3 & 7 & 2 & 5 & 2 & 2 & e & & $\mathbf{E}$ & United States & & RM1018 \\
\hline$F 2 a$ & 192 & 784 & 9 & 5 & 9 & 2 & 5 & 1 & 6 & 2 & 2 & 2 & 3 & 3 & 7 & 2 & 5 & 2 & 2 & e & & & United States & 1983 & RM8031 \\
\hline $\mathbf{F} 2 \mathbf{a}$ & 193 & 634 & 9 & 5 & 9 & 2 & 5 & 1 & 6 & 2 & 4 & 2 & 3 & 5 & 7 & 2 & 5 & 2 & 2 & e & & E & England & 1967 & RM6200 \\
\hline F2a & 194 & 755 & 9 & 5 & 9 & 2 & 5 & 3 & 6 & 2 & 2 & 2 & 3 & 6 & 7 & 2 & 5 & 2 & 2 & e & & $\mathbf{E}$ & Malaysia & 1973 & RM7279 \\
\hline F2a & 195 & 585 & 9 & 7 & 9 & 2 & 5 & 1 & 3 & 2 & 4 & 2 & 3 & 3 & 7 & 2 & 5 & 2 & 2 & e & & & Unknown & & RM6151 \\
\hline F2a & 196 & 644 & 9 & 0 & 9 & 2 & 5 & 1 & 3 & 2 & 4 & 2 & 3 & 3 & 7 & 2 & 5 & 2 & 2 & e & & E & England & 1972 & RM6210 \\
\hline F2a & 197 & 592 & 9 & 5 & 9 & 2 & 5 & 1 & 3 & 2 & 4 & 2 & 3 & 3 & 7 & 2 & 5 & 2 & 2 & $\mathrm{e}$ & & & England & 1962 & RM6158 \\
\hline F2a & 198 & 596 & 9 & 5 & 9 & 2 & 5 & 1 & 3 & 2 & 4 & 2 & 3 & 3 & 8 & 2 & 5 & 2 & 2 & e & & E & England & 1963 & RM6162 \\
\hline F2a & 199 & 589 & 9 & 5 & 9 & 2 & 5 & 1 & 3 & 2 & 4 & 2 & 3 & 3 & 7 & 2 & 5 & 3 & 2 & e & & E & Unknown & & RM6155 \\
\hline $\mathbf{F} 2 \mathbf{a}$ & 200 & 590 & 9 & 5 & 9 & 2 & 5 & 1 & 3 & 2 & 4 & 2 & 2 & 3 & 6 & 2 & 5 & 2 & 2 & e & & E & Unlnown & & RM6156 \\
\hline F2a & 201 & 624 & 9 & 5 & 9 & 2 & 5 & 1 & 3 & 2 & 4 & 2 & 2 & 3 & 7 & 2 & 5 & 2 & 2 & e & & E & England & 1966 & RM6190 \\
\hline $\mathbf{F} 2 \mathrm{~b}$ & 202 & 615 & 5 & 5 & 9 & 2 & 5 & 1 & 5 & 2 & 4 & 2 & 3 & 3 & 7 & 2 & 1 & 2 & 2 & e & & Anom** & England & 1965 & RM6181 \\
\hline $\mathbf{F} 2 \mathbf{b}$ & 203 & 709 & 5 & 5 & 9 & 2 & 5 & 1 & 3 & 2 & 1 & 2 & 3 & 3 & 7 & 2 & 1 & 2 & 2 & e & & E & Papua New Guinea & $1980 \mathrm{~s}$ & RM7066 \\
\hline $\mathbf{F} 2 \mathbf{c}$ & 204 & 601 & 9 & 5 & 9 & 2 & 5 & 3 & 3 & 2 & 2 & 2 & 3 & 3 & 7 & 2 & 5 & 2 & 2 & e & & $\mathbf{E}$ & England & 1963 & RM6167 \\
\hline F $2 \mathrm{c}$ & 205 & 1796 & 9 & 5 & 9 & 2 & 5 & 3 & 3 & 2 & 2 & 2 & 3 & 3 & 5 & 2 & 5 & 2 & 2 & e & & & Kenya & 1986 & RM7415 \\
\hline $\mathbf{F} 2 \mathrm{c}$ & 206 & 756 & 9 & 5 & 9 & 2 & 4 & 3 & 3 & 2 & 2 & 2 & 3 & 3 & 7 & 2 & 5 & 2 & 2 & e & & E & Malaysia & 1973 & RM7280 \\
\hline $\mathbf{F} 2 \mathrm{c}$ & 207 & 600 & 9 & 5 & 9 & 2 & 5 & 3 & 3 & 2 & 2 & 2 & 3 & 4 & 7 & 2 & 5 & 2 & 2 & e & & E & England & 1963 & RM6166 \\
\hline$F 2 c$ & 208 & 612 & 9 & 5 & 9 & 2 & 5 & 3 & 3 & 2 & 2 & 2 & 3 & 3 & 7 & 2 & 5 & 2 & 1 & e & & E & England & 1964 & RM6178 \\
\hline $\mathbf{F} 2 \mathrm{c}$ & 209 & 611 & 9 & 0 & 9 & 2 & 5 & 3 & 3 & 2 & 2 & 2 & 3 & 3 & 7 & 2 & 5 & 2 & 1 & e & & & England & 1964 & RM6177 \\
\hline F2c & 210 & 645 & 9 & 0 & 9 & 2 & 5 & 3 & 3 & 2 & 2 & 2 & 3 & 3 & 7 & 2 & 5 & 2 & 2 & e & & E & England & 1972 & RM6211 \\
\hline F2c & 211 & 662 & 0 & 0 & 9 & 2 & 5 & 3 & 3 & 2 & 2 & 2 & 3 & 3 & 7 & 2 & 5 & 2 & 2 & e & & E & England & 1976 & RM6228 \\
\hline $\mathrm{F} 2 \mathrm{c}$ & 212 & 649 & 9 & 8 & 9 & 2 & 5 & 3 & 3 & 2 & 2 & 2 & 3 & 3 & 7 & 2 & 5 & 2 & 2 & e & & & England & 1973 & RM6215 \\
\hline $\mathrm{F} 2 \mathrm{c}$ & 213 & 763 & 9 & 5 & 9 & 2 & 5 & 3 & 3 & 2 & 2 & 2 & 3 & 5 & 6 & 2 & 5 & 2 & 2 & e & & & Malaysia & $1970 \mathrm{~s}$ & RM7287 \\
\hline$F 2 \mathfrak{c}$ & 214 & 642 & 9 & 5 & 9 & 2 & 3 & 4 & 3 & 2 & 2 & 2 & 3 & 3 & 7 & 2 & 5 & 2 & 2 & e & & E & England & 1971 & RM6208 \\
\hline$F 2 \mathfrak{c}$ & 215 & 663 & 5 & 0 & 10 & 2 & 5 & 3 & 3 & 2 & 2 & 2 & 3 & 3 & 7 & 2 & 5 & 2 & 2 & e & & E & England & 1977 & RM6229 \\
\hline$F 2 c$ & 216 & 653 & 5 & 5 & 10 & 2 & 5 & 3 & 3 & 2 & 2 & 2 & 3 & 3 & 7 & 2 & 5 & 2 & 2 & $\mathbf{e}$ & & $\mathbf{E}$ & England & 1974 & RM6219 \\
\hline$F 2 c$ & 217 & 661 & 0 & 5 & 10 & 2 & 5 & 3 & 3 & 2 & 2 & 2 & 3 & 3 & 7 & 2 & 5 & 2 & 2 & e & & E & England & 1976 & RM6227 \\
\hline G1 & 218 & 1804 & 5 & 5 & 9 & 2 & 2 & 1 & 5 & 2 & 2 & 2 & 3 & 3 & 4 & 2 & 2 & 2 & 3 & e & & E & Kenya & 1986 & RM7423 \\
\hline $\mathrm{Hla}$ & 219 & 515 & 7 & 5 & 4 & 1 & 3 & 1 & 3 & 2 & 5 & 2 & 3 & 2 & 6 & 3 & 1 & 2 & 2 & a & $2 \mathrm{H}$ & $\mathbf{M}$ & Unknown & & RM1147 \\
\hline $\mathrm{Hla}$ & 220 & 553 & 7 & 5 & 4 & 1 & 5 & 1 & 3 & 2 & 5 & 2 & 3 & 2 & 6 & 3 & 1 & 2 & 2 & a & $4 \mathrm{H}$ & M & England & 1977 & RM6083 \\
\hline $\mathrm{Hla}$ & 221 & 543 & 7 & 5 & 4 & 1 & 3 & 1 & 3 & 2 & 5 & 2 & 2 & 2 & 6 & 3 & 1 & 2 & 2 & a & $2 \mathrm{H}$ & M & England & 1966 & RM6073 \\
\hline $\mathrm{Hlb}$ & 222 & 529 & 7 & 4 & 4 & 1 & 5 & ] & 3 & 2 & 5 & 2 & 3 & 3 & 4 & 3 & 1 & 2 & 2 & a & $6 \mathrm{H}$ & M & England & 1964 & RM6059 \\
\hline HIb & 223 & 534 & 7 & 5 & 4 & 1 & 5 & 1 & 3 & 2 & 5 & 2 & 3 & 3 & 4 & 3 & 1 & 2 & 2 & a & $6 \mathrm{H}$ & $\mathbf{M}$ & England & 1966 & RM6064 \\
\hline $\mathrm{Hlb}$ & 224 & 540 & 7 & 4 & 4 & 1 & 5 & 1 & 3 & 2 & 5 & 2 & 3 & 2 & 4 & 3 & 1 & 2 & 2 & a & $6 \mathrm{H}$ & $\mathbf{M}$ & England & 1962 & RM6070 \\
\hline Hib & 225 & 549 & 0 & 4 & 4 & 1 & 5 & 1 & 3 & 2 & 5 & 2 & 3 & 2 & 4 & 3 & 1 & 2 & 2 & a & $6 \mathrm{H}$ & $\mathbf{M}$ & England & 1967 & RM6079 \\
\hline Hlb & 226 & 538 & 7 & 5 & 4 & 1 & 5 & 1 & 3 & 2 & 5 & 2 & 3 & 2 & 4 & 4 & 1 & 2 & 2 & $\mathbf{a}$ & $6 \mathrm{H}$ & $\mathbf{M}$ & England & 1968 & RM6068 \\
\hline Ila & 227 & 532 & 1 & 5 & 5 & 1 & 5 & 1 & 2 & 1 & 5 & 2 & 3 & 1 & 4 & 2 & 4 & 2 & 2 & a & $4 \mathrm{H}$ & M & England & 1965 & RM6062 \\
\hline $\mathrm{IIa}$ & 228 & 539 & 1 & 5 & 5 & 1 & 5 & $\mathbf{I}$ & 2 & 1 & 5 & 2 & 3 & 1 & 4 & 3 & 4 & 2 & 2 & a & $4 \mathrm{H}$ & $\mathbf{M}$ & England & 1963 & RM6069 \\
\hline 11a & 229 & 550 & 1 & 5 & 5 & 1 & 5 & 1 & 2 & 1 & 5 & 1 & 3 & $i$ & 4 & 2 & 4 & 2 & 2 & a & $4 \mathrm{H}$ & $\mathbf{M}$ & England & 1967 & RM6080 \\
\hline $11 \mathrm{~b}$ & 230 & 724 & 1 & 5 & 4 & 1 & 5 & 1 & 3 & 1 & 4 & 2 & 3 & 1 & 4 & 2 & 4 & 2 & 2 & a & $5 \mathrm{H}$ & $\mathbf{M}$ & Dominican Republic & $1980 \mathrm{~s}$ & RM7115 \\
\hline Jla & 231 & 3106 & 7 & 5 & 4 & 2 & 5 & 1 & 4 & 1 & 2 & 3 & 5 & 2 & 2 & 5 & 4 & 2 & 2 & b & & & Uniled States & 1968 & CATLIN \\
\hline Jla & 232 & 3107 & 7 & 5 & 4 & 2 & 5 & 1 & 4 & 1 & 5 & 3 & 5 & 2 & 2 & 5 & 4 & 2 & 2 & $b$ & & o & United States & 1968 & CATLIN 5788 \\
\hline $\mathrm{Jlb}$ & 233 & 1076 & 7 & 5 & 4 & 2 & 5 & 1 & 3 & 1 & 4 & 2 & 5 & 2 & 1 & 3 & 4 & 2 & 2 & b & $8-\mathrm{H}^{\dagger \dagger}$ & & United States & 1983 & SLCH 3491 \\
\hline Jlb & 234 & 3108 & 7 & 6 & 4 & 2 & 5 & 1 & 3 & 1 & 4 & 3 & 5 & 2 & 1 & 3 & 4 & 2 & 2 & b & $8 \mathbf{H}$ & & Alaska & $1980 \mathrm{~s}$ & JW 28 \\
\hline $\mathrm{Jlb}$ & 235 & 3109 & 7 & 7 & 4 & 2 & 5 & 1 & 3 & 1 & 4 & 3 & 5 & 2 & 1 & 3 & 4 & 2 & 2 & b & $8 \mathbf{H}$ & $\mathbf{o}$ & Alaska & 1980 & JW 43 \\
\hline $\mathrm{Jtb}$ & 236 & 1069 & 3 & 5 & 4 & 2 & 5 & 1 & 3 & 1 & 3 & 3 & 5 & 2 & 1 & 3 & 4 & 2 & 2 & b & $8 \mathbf{H}$ & & United States & 1954 & SLCH 3191 \\
\hline $\mathrm{Jlb}$ & 237 & 1070 & 7 & 5 & 4 & 2 & 5 & 1 & 3 & 1 & 3 & 3 & 5 & 2 & 1 & 3 & 4 & 3 & 2 & b & $8 \mathbf{H}$ & & United States & 1940 & SLCH 3205 \\
\hline J2 & 238 & 1303 & 7 & 5 & 4 & 5 & 5 & 1 & 3 & 1 & 4 & 4 & 5 & 2 & 2 & 2 & 4 & 2 & 2 & $b$ & & & South Africa & $1980 \mathrm{~s}$ & CAPE TOWN 8811 \\
\hline J3 & 239 & 1084 & 7 & 5 & 4 & 3 & 5 & 1 & 7 & 1 & 4 & 2 & 1 & 3 & 2 & 3 & 4 & 2 & 2 & $b$ & $17 \mathrm{H}$ & & United States & 1947 & RABINOWITZ \\
\hline Kla & 240 & 511 & 0 & 4 & 3 & 2 & 5 & 1 & 3 & 1 & 5 & 2 & 2 & 3 & 4 & 3 & 4 & 2 & 2 & $\mathrm{f}$ & & 0 & United States & 1979 & RM1121 \\
\hline
\end{tabular}


Table 2. (continued)

\begin{tabular}{|c|c|c|c|c|c|c|c|c|c|c|c|c|c|c|c|c|c|c|c|c|c|c|c|c|c|}
\hline & & & & & & & & & llele a & indic & cated er & enzyme & e locus & & & & & & & & OMP & & & & \\
\hline Group & ET & no.* & CAK & NSP & PGI & MAE & MDH & G6P & GOT & ADK & 6PG & PE2 1 & PEL & LAP I & PGM & CAT & GLD & G3P F & FUM & type & type & pattern & source & isolation & Other designation \\
\hline K1b & 241 & 686 & 7 & 5 & 3 & 2 & 3 & 1 & 3 & 1 & 5 & 2 & 2 & 3 & 7 & 3 & 4 & 2 & 2 & f & & & England & 1966 & RM6252 \\
\hline K1e & 242 & 697 & 7 & 5 & 2 & 2 & 5 & 1 & 3 & 1 & 5 & 2 & 2 & 2 & 7 & 3 & 4 & 2 & 2 & $f$ & & F & England & & RM6263 \\
\hline $\mathrm{K} 1 \mathrm{c}$ & 243 & 698 & 7 & 5 & 2 & 2 & 5 & 1 & 3 & 1 & 5 & 2 & 2 & 3 & 7 & 3 & 4 & 2 & 2 & f & & & England & 1984 & RM6265 \\
\hline K1c & 244 & 771 & 7 & 5 & 2 & 2 & 5 & 1 & 3 & 1 & 5 & 2 & 2 & 3 & 2 & 3 & 4 & 2 & 2 & f & & F & Malaysia & $1970 \mathrm{~s}$ & RM7299 \\
\hline Klc & 245 & 757 & 7 & 5 & 2 & 2 & 5 & 1 & 3 & 1 & 5 & 2 & 2 & 3 & 4 & 3 & 4 & 2 & 2 & f & & & Malaysia & 1971 & RM7281 \\
\hline Klc & 246 & 758 & 7 & 5 & 2 & 2 & 5 & 1 & 3 & 1 & 5 & 2 & 2 & 2 & 4 & 3 & 4 & 2 & 2 & f & & & Malaysia & $1970 \mathrm{~s}$ & RM7282 \\
\hline $\mathrm{K} 1 \mathbf{c}$ & 247 & 768 & 7 & 5 & 2 & 2 & 5 & 1 & 3 & 1 & 5 & 2 & 2 & 5 & 4 & 3 & 4 & 2 & 2 & f & & & Malaysia & $1970 \mathrm{~s}$ & RM7295 \\
\hline $\mathrm{K} 1 \mathrm{c}$ & 248 & 761 & 7 & 5 & 2 & 2 & 5 & 1 & 3 & 1 & 5 & 2 & 2 & 6 & 4 & 3 & 4 & 2 & 2 & f & & & Malaysia & $1970 \mathrm{~s}$ & RM7285 \\
\hline K1c & 249 & 759 & 7 & 5 & 2 & 2 & 5 & 1 & 3 & 1 & 5 & 2 & 2 & 1 & 4 & 3 & 4 & 2 & 2 & f & & $\mathbf{F}$ & Malaysia & $1970 \mathrm{~s}$ & RM7283 \\
\hline K1c & 250 & 770 & 7 & 5 & 2 & 2 & 5 & 1 & 3 & 1 & 5 & 2 & 2 & 1 & 2 & 3 & 4 & 2 & 2 & $f$ & & $\mathrm{~F}$ & Malaysia & $1970 \mathrm{~s}$ & RM7298 \\
\hline Kle & 251 & 764 & 5 & 5 & 2 & 2 & 5 & 1 & 3 & 1 & 5 & 2 & 2 & 5 & 4 & 3 & 4 & 2 & 2 & f & & F & Malaysia & $1970 \mathrm{~s}$ & RM7290 \\
\hline $\mathbf{K} \mathbf{l c}$ & 252 & 760 & 7 & 0 & 2 & 2 & 5 & 1 & 3 & 1 & 5 & 2 & 2 & 1 & 4 & 3 & 4 & 2 & 2 & f & & & Malaysia & $1970 \mathrm{~s}$ & RM7284 \\
\hline $\mathbf{K} 1 \mathrm{c}$ & 253 & 762 & 7 & 0 & 2 & 2 & 5 & 1 & 3 & 1 & 5 & 2 & 2 & 3 & 4 & 3 & 4 & 2 & 2 & $f$ & & $\mathrm{~F}$ & Malaysia & $1970 \mathrm{~s}$ & RM7286 \\
\hline $\mathbf{K} 1 \mathbf{c}$ & 254 & 765 & 5 & 0 & 2 & 2 & 5 & 1 & 3 & 1 & 5 & 2 & 2 & 6 & 4 & 3 & 4 & 2 & 2 & $\mathrm{f}$ & & F & Malaysia & $1970 \mathrm{~s}$ & RM7292 \\
\hline K1d & 255 & 776 & 7 & 0 & 2 & 5 & 5 & 1 & 3 & 1 & 5 & 2 & 2 & 3 & 2 & 3 & 4 & 2 & 2 & f & & & United States & 1984 & RM8009 \\
\hline $\mathbf{K} 2 \mathrm{a}$ & 256 & 514 & 7 & 5 & 3 & 2 & 5 & 3 & 4 & 1 & 5 & 2 & 2 & 3 & 6 & 3 & 1 & 2 & 2 & $\mathrm{f}$ & & 0 & United States & & RM1137 \\
\hline $\mathbf{K} 2 \mathrm{a}$ & 257 & 689 & 7 & 5 & 3 & 2 & 5 & 3 & 4 & 1 & 5 & 2 & 2 & 3 & 7 & 3 & 1 & 2 & 2 & f & & & England & 1967 & RM6255 \\
\hline $\mathbf{K} 2 \mathrm{a}$ & 258 & 699 & 7 & 5 & 3 & 2 & 5 & 3 & 4 & 1 & 5 & 2 & 2 & 2 & 7 & 3 & 1 & 2 & 2 & $f$ & & UN辡 & England & 1984 & RM6266 \\
\hline $\mathbf{K} 2 \mathrm{a}$ & 259 & 693 & 7 & 5 & 3 & 2 & 5 & 3 & 4 & 1 & 5 & 1 & 2 & 3 & 7 & 3 & 1 & 2 & 2 & f & & 01 & England & 1967 & RM6259 \\
\hline $\mathbf{K} 2 \mathrm{a}$ & 260 & 688 & 7 & 0 & 3 & 2 & 5 & 3 & 4 & 1 & 5 & 2 & 2 & 3 & 7 & 3 & 1 & 2 & 2 & f & & 0 & England & 1966 & RM6254 \\
\hline $\mathrm{K} 2 \mathrm{a}$ & 261 & 696 & 7 & 0 & 3 & 2 & 5 & 3 & 4 & 1 & 5 & 2 & 2 & 2 & 7 & 3 & 1 & 2 & 2 & f & & 01 & England & 1968 & RM6262 \\
\hline $\mathrm{K} 2 \mathrm{a}$ & 262 & 669 & 7 & 5 & 3 & 2 & 5 & 3 & 4 & 1 & 5 & 2 & 1 & 3 & 6 & 3 & 1 & 2 & 2 & f & & 01 & England & 1963 & RM6235 \\
\hline $\mathrm{K} 2 \mathrm{a}$ & 263 & 678 & 7 & 5 & 3 & 2 & 5 & 3 & 4 & 1 & 5 & 2 & 1 & 3 & 7 & 3 & 1 & 2 & 2 & $\mathbf{f}$ & & 0 & England & 1964 & RM6244 \\
\hline $\mathrm{K} 2 \mathrm{a}$ & 264 & 673 & 7 & 5 & 3 & 2 & 5 & 3 & 4 & 1 & 5 & 2 & 1 & 5 & 6 & 3 & 1 & 2 & 2 & f & & UN & England & 1963 & RM6239 \\
\hline $\mathrm{K} 2 \mathrm{a}$ & 265 & 671 & 7 & 0 & 3 & 2 & 5 & 3 & 4 & 1 & 5 & 2 & 1 & 3 & 6 & 3 & 1 & 2 & 2 & f & & 0 & England & 1963 & RM6237 \\
\hline $\mathrm{K} 2 \mathrm{a}$ & 266 & 679 & 7 & 0 & 3 & 2 & 5 & 3 & 4 & 1 & 5 & 2 & 1 & 3 & 7 & 3 & 1 & 2 & 2 & f & & & England & 1964 & RM6245 \\
\hline $\mathrm{Ll}$ & 267 & 1798 & 7 & 5 & 3 & 2 & 5 & 1 & 4 & 1 & 1 & 2 & 2 & 5 & 5 & 2 & 1 & 3 & 2 & $\mathbf{f}$ & & & Kenya & 1986 & RM7417 \\
\hline
\end{tabular}

NOTE. See text or footnote to table 3 for enzyme abbreviations

* Musser Haemophilus.

$\uparrow$ Pennington.

¥ Similar to subtype 1L, but not identical.

$\$$ Johannesburg.

"Children's Hospital of Philadelphia.

I Prince Edward Island.

** Anomalous.

†† Similar to subtype 8, but apparently lacks P1 band.

柿 Undefined.

\section{Genetic Relationships Among Multilocus Enzyme Genotypes}

The dendrogram in figure 1 summarizes estimates of the genetic relationships of the $280 \mathrm{ETs}$, based on allelic variation at the 17 enzyme loci. At a genetic distance of 0.20 , there were 56 branches, each represented by a single ET or a cluster of ETs. Twelve major lineages diverging at distances greater than 0.42 were designated by the capital letters $A$ through $L$; clusters of ETs in these lineages were numbered (e.g., $A 1, A 2$, etc.), and groups of ETs in a cluster were designated with lower case letters. Lineages A through $\mathrm{G}$ were separated from lineages $\mathrm{H}$ through $L$ at a genetic distance of 0.66 , which means that ETs in these two primary divisions of the dendrogram (I and II) differed, on average, at 10 of the 17 loci assayed.
Major lineage A, in division I, was composed entirely of ETs of serotype b isolates. (Cluster Al and clusters A2 through A5 included ETs previously assigned by Musser et al. [20, 29] to clone families [groups of closely allied clones] designated as $A$ and B, respectively.) Lineage B, which diverged from lineage $A$ at a genetic distance of 0.43 , contained ETs of isolates producing capsule types a, b, and d. (Serotype $b$ isolates in this lineage previously were assigned to clone family C $[20,29]$.) Clusters D1 through E1 contained the ETs of all the serotype $c$ isolates studied, and lineages $F$ and $G$, which were separated from lineages $\mathrm{A}$ through $\mathrm{E}$ at a genetic distance of 0.58 , included all ETs represented by serotype e isolates. Lineages $\mathrm{H}$ and $\mathrm{I}$, clusters $\mathrm{J} 1$ through $\mathrm{J} 3$, and clusters K1 through L1 included ETs of isolates of serotype $\mathrm{a}, \mathrm{b}$, and $\mathrm{f}$, respectively. (Serotype b strains in clusters 
Table 3. Mean genetic diversity $(H)$ at 17 enzyme loci in 280 ETs of encapsulated $H$. influenzae.

\begin{tabular}{|c|c|c|c|c|c|c|c|}
\hline \multirow{3}{*}{$\begin{array}{l}\text { Enzyme } \\
\text { locus* }\end{array}$} & \multicolumn{7}{|c|}{ Mean genetic diversity (no. of alleles) } \\
\hline & \multicolumn{6}{|c|}{ Serotype } & \multirow[b]{2}{*}{ Total } \\
\hline & a & b & c & d & $\mathrm{e}$ & $\mathbf{f}$ & \\
\hline CAK & $0.700(4)$ & $0.808(9)$ & $0.182(2)$ & $0.000(1)$ & $0.486(3)$ & $0.204(3)$ & $0.812(9)$ \\
\hline NSP & $0.257(2)$ & $0.224(5)$ & $0.509(2)$ & $0.000(1)$ & $0.385(4)$ & $0.474(3)$ & $0.286(6)$ \\
\hline PGI & $0.567(3)$ & $0.606(6)$ & $0.600(4)$ & $0.000(1)$ & $0.181(2)$ & $0.519(2)$ & $0.770(11)$ \\
\hline MAE & $0.552(3)$ & $0.398(5)$ & $0.000(1)$ & $0.286(2)$ & $0.000(1)$ & $0.071(2)$ & $0.623(6)$ \\
\hline MDH & $0.343(3)$ & $0.391(5)$ & $0.509(2)$ & $0.000(1)$ & $0.187(4)$ & $0.071(2)$ & $0.358(6)$ \\
\hline G6P & $0.514(2)$ & $0.532(6)$ & $0.600(4)$ & $0.000(1)$ & $0.630(4)$ & $0.495(2)$ & $0.647(8)$ \\
\hline GOT & $0.538(4)$ & $0.501(6)$ & $0.345(3)$ & $0.571(2)$ & $0.340(3)$ & $0.508(2)$ & $0.538(7)$ \\
\hline ADK & $0.324(2)$ & 0.135 & 0.000 & 0.000 & 0.000 & 0.000 & 0.274 (4) \\
\hline $6 \mathrm{PG}$ & $0.567(3)$ & $0.260(5)$ & $0.000(1)$ & $0.000(1)$ & $0.495(3)$ & $0.071(2)$ & $0.449(5)$ \\
\hline PE2 & 0.552 & $0.214(5)$ & $0.345(3)$ & $0.476(2)$ & $0.181(2)$ & $0.071(2)$ & $0.485(6)$ \\
\hline PE1 & $0.605(4)$ & $0.147(6)$ & $0.855(5)$ & $0.000(1)$ & $0.185(3)$ & $0.304(2)$ & $0.526(8)$ \\
\hline LAP & $0.748(5)$ & $0.661(6)$ & $0.836(5)$ & $0.286(2)$ & $0.243(4)$ & $0.680(5)$ & $0.731(8)$ \\
\hline PGM & $0.652(4)$ & $0.355(4)$ & $0.473(3)$ & $0.286(2)$ & $0.299(5)$ & $0.738(5)$ & $0.589(8)$ \\
\hline CAT & $0.552(3)$ & $0.135(4)$ & $0.000(1)$ & $0.286(2)$ & 0.000 (1) & $0.071(2)$ & $0.300(5)$ \\
\hline GLD & $0.495(2)$ & $0.044(4)$ & $0.000(1)$ & $0.286(2)$ & $0.288(3)$ & $0.508(2)$ & $0.345(6)$ \\
\hline G3P & $0.095(2)$ & $0.065(3)$ & $0.436(2)$ & $0.286(2)$ & $0.065(2)$ & $0.071(2)$ & $0.090(3)$ \\
\hline FUM & $0.467(2)$ & 0.043 & $0.473(3)$ & $0.000(1)$ & $0.185(3)$ & $0.000(1)$ & $0.116(3)$ \\
\hline Average & $0.502(3.0)$ & $0.325(5.1)$ & $0.363(2.5)$ & $0.162(1.5)$ & $0.242(2.8)$ & $0.286(2.4)$ & $0.467(6.4)$ \\
\hline
\end{tabular}

* Abbreviations: CAK, carbamylate kinase; NSP, nucleoside phosphorylase; PGI, phosphoglucose isomerase; MAE, malic enzyme; MDH, malate dehydrogenase; G6P, glucose 6-phosphate dehydrogenase; GOT, glutamic oxaloacetic transaminase; ADK, adenylate kinase; 6PG, 6-phosphogluconate dehydrogenase; PE2, leucylalanine peptidase-2; PE1, leucylalanine peptidase-1; LAP, leucine aminopeptidase; PGM, phosphoglucomutase; CAT, catalase; GLD, glutamate dehydrogenase; G3P, glyceraldehyde-3-phosphate dehydrogenase; and FUM, fumarase.

$\mathrm{J} 1$ through $\mathrm{J} 3$ previously were assigned to clone family D [29].)

In summary, multilocus enzyme electrophoresis revealed two major genetic divisions among encapsulated $H$. influenzae. Division I contained all ETs of serotype $c$ and $d$ strains, one phylogenetic line of serotype a strains, and most ETs $(\mathbf{9 5 \%})$ and isolates (99\%) of serotype b. Division II included all ETs of serotype $f$, a second major line of ETs expressing serotype a capsule, and a genetically heterogeneous second group of ETs of serotype $b$ isolates. Thus, ETs of isolates of serotypes $a$ and $b$ occurred in each of the two primary phylogenetic divisions that are separated at a genetic distance of 0.66 .

The allele profiles and additional characteristics of representative isolates of the 280 ETs of encapsulated $H$. influenzae are presented in table 2; information on the geographic origin, OMP type, and cap region RFLP pattern of the isolates assigned to each ET is given in table 4. ETs were numbered in consecutive order according to their positions in the dendrogram (figure 1), with the exception of ETs that were identical at all loci except the CAK locus to the four common multilocus genotypes (ETs 1, 12, 21, and 25) earlier identified by Musser et al. [20], for which are reserved the numbers previously assigned. After the appearance of the paper describing these four ETs [20], two additional enzymes were identified, carbamylate kinase and nucleoside phosphorylase, that can be assayed in $\boldsymbol{H}$. influenzae isolates. Carbamylate kinase is strongly polymorphic in serotype $b$ isolates, and many electrophoretic variants of this enzyme are represented among isolates previously grouped in each of the four common ETs (ETs 1, 12, 21, and 25). Consequently, isolates of each of these ETs are now differentiated into a series of ETs on the basis of alleles of CAK (e.g., ET 12.5, $12.6,12.7,12.8$, etc.).

The great majority $(80 \%)$ of serotype b ETs are in groups $\mathrm{Ala}, \mathrm{Alc}, \mathrm{A2a}, \mathrm{A2e}$, and B1b, and most isolates are of ETs in groups Ala (28\%), A2a $(60 \%)$, and Blb (6\%). The four ETs represented by the largest number of isolates are ET $1.9(81 \%$ of isolates in group Ala), ET 12.5 and 12.8 (75\% of isolates in group A2a), and ET $25.6(40 \%$ of isolates in group $\mathrm{B} 1 \mathrm{~b}$ ). Isolates of these four multilocus geno- 
Table 4. Characteristics of 280 ETs (represented by 2,209 isolates) of encapsulated $H$. influenzae.

\begin{tabular}{|c|c|c|c|}
\hline $\begin{array}{l}\text { Group } \\
\text { ET }\end{array}$ & $\begin{array}{c}\text { Geographic source } \\
\text { (no. of isolates) }\end{array}$ & $\begin{array}{c}\text { OMP } \\
\text { pattern* }\end{array}$ & $\begin{array}{c}\text { cap } \\
\text { region } \\
\text { RFLP } \\
\text { pattern }\end{array}$ \\
\hline Ala 1.9 & Alaska (26) & $\begin{array}{l}1 \mathrm{H}(13), 2 \mathrm{~L}(6) \\
2 \mathrm{H}(4), 1 \mathrm{~L}(2), \\
4 \mathrm{H}(1)\end{array}$ & \\
\hline Ala 1.9 & Australia (3) & & \\
\hline Ala 1.9 & Canada (143) & $\begin{array}{l}2 \mathrm{~L}(22), 1 \mathrm{~L}(2) \\
\quad 1 \mathrm{H}(2), 19 \mathrm{H}(1)\end{array}$ & \\
\hline Ala 1.9 & Denmark (3) & $1 L(2)$ & \\
\hline Ala 1.9 & Dominican Republic (1) & & $S(1)$ \\
\hline Ala 1.9 & England (3) & & $\mathrm{G}(2)$ \\
\hline Ala 1.9 & Finland (2) & & \\
\hline Ala 1.9 & France (1) & & \\
\hline Ala 1.9 & Hawaii (7) & $1 \mathrm{H}(1), 11 \mathrm{~L}(1)$ & \\
\hline Ala 1.9 & Iceland (33) & $\begin{array}{c}2 \mathrm{~L}(31), 1 H(1) \\
G(1), S(1) \\
V(1)\end{array}$ & \\
\hline Ala 1.9 & Mexico (1) & & \\
\hline Ala 1.9 & Norway (6) & $1 \mathrm{H}(4), 2 \mathrm{H}(1)$ & \\
\hline Ala 1.9 & Papua New Guinea (1) & & \\
\hline Ala 1.9 & South Africa (2) & $2 \mathrm{~L}(2)$ & $S(1)$ \\
\hline Ala 1.9 & Sweden $(6)$ & & $G(1)$ \\
\hline Ala 1.9 & Switzerland (2) & $1 \mathrm{H}(1), 2 \mathrm{~L}(1)$ & \\
\hline Ala 1.9 & United States (210) & $\begin{array}{l}1 \mathrm{H}(47), 2 \mathrm{~L}(25) \\
2 \mathrm{H}(4), 7 \mathrm{H}(1), \\
10 \mathrm{H}(1), 15 \mathrm{~L}(1), \\
18 \mathrm{~L}(1), \mathrm{V}(25), \\
\mathrm{G}(5)\end{array}$ & \\
\hline Ala 2 & Canada (4) & $1 H(1)$ & \\
\hline A1a 2 & United States (1) & $1 H(1)$ & \\
\hline Ala 3 & United States (1) & & \\
\hline Ala 4 & Alaska (6) & $1 \mathrm{~L}(6)$ & \\
\hline Ala 4 & Canada (5) & & \\
\hline Ala 4 & Iceland (1) & & \\
\hline Ala 4 & Sweden (1) & & \\
\hline Ala 4 & United States (19) & $1 \mathrm{~L}(11), 1 \mathrm{H}(2)$ & $G(1)$ \\
\hline Ala 5 & United States (1) & $1 \mathrm{H}(1)$ & $\mathrm{G}(1)$ \\
\hline Ala 6 & United States (1) & $1 L(1)$ & $G(1)$ \\
\hline Ala 7 & United States (1) & $1 \mathrm{~L}(1)$ & \\
\hline Ala 1.6 & Canada (2) & & \\
\hline Ala 1.6 & Alaska (1) & $2 \mathrm{~L}(1)$ & \\
\hline Ala 1.7 & Australia (1) & & $\mathrm{V}(1)$ \\
\hline Ala 1.8 & Norway (1) & $1 \mathrm{~L}(1)$ & \\
\hline Ala 8 & Australia (1) & & \\
\hline Ala 8 & Hawaii (1) & & \\
\hline Ala 8 & Kenya (4) & $3 L(3)$ & $G(4)$ \\
\hline Ala 8 & South Africa (5) & & \\
\hline A1a 8 & Switzerland (1) & & \\
\hline Ala 9 & Canada (1) & & \\
\hline Ala 10 & United States (1) & & \\
\hline Ala 11 & United States (1) & $1 L(1)$ & \\
\hline Ala 13 & United States (2) & $1 \mathrm{H}(1), 2 \mathrm{~L}(1)$ & \\
\hline Ala 14 & Alaska (1) & IH(1) & \\
\hline Ala 15 & United States (1) & $2 L(1)$ & \\
\hline Ala 16 & Canada (2) & & \\
\hline
\end{tabular}

Table 4. (continued)

\begin{tabular}{|c|c|c|c|}
\hline $\begin{array}{l}\text { Group } \\
\text { ET }\end{array}$ & $\begin{array}{l}\text { Geographic source } \\
\text { (no. of isolates) }\end{array}$ & $\begin{array}{c}\text { OMP } \\
\text { pattern* }\end{array}$ & $\begin{array}{c}\text { cap } \\
\text { region } \\
\text { RFLP } \\
\text { pattern }\end{array}$ \\
\hline Ala 16 & England (1) & & \\
\hline A1a 16 & United States (1) & & \\
\hline Ala 17 & Canada (2) & & \\
\hline Ala 18 & Alaska (1) & $2 \mathrm{~L}(1)$ & \\
\hline Ala 19 & Alaska (3) & $2 L(3)$ & \\
\hline Ala 20 & Canada (1) & & \\
\hline Ala 20 & United States (1) & $1 \mathrm{H}(1)$ & \\
\hline Ala 22 & Canada (1) & & \\
\hline A1a 22 & The Netherlands (1) & & \\
\hline Ala 22 & Scotland (1) & & \\
\hline Ala 22 & United States (2) & $2 \mathrm{~L}(2)$ & \\
\hline Ala 23 & Scotland (1) & & \\
\hline Ala 24 & United States (1) & $2 \mathrm{~L}(1)$ & \\
\hline Ala 26 & United States (1) & $1 \mathrm{H}(1)$ & $G(1)$ \\
\hline A1a 27 & United States (1) & $1 \mathrm{~L}(1)$ & \\
\hline Ala 28 & United States (1) & & \\
\hline Ala 29 & United States (1) & $2 \mathrm{~L}(1)$ & $\mathrm{V}(1)$ \\
\hline Ala 30 & United States (2) & $1 \mathrm{H}(1), 9 \mathrm{~L}(1)$ & $\mathrm{V}(1)$ \\
\hline Ala 31 & England (1) & & $G(1)$ \\
\hline Ala 32 & Canada (1) & & \\
\hline Ala 32 & England (1) & & $G(1)$ \\
\hline Ala 32 & United States (3) & $1 \mathrm{~L}(1), 2 \mathrm{H}(1)$ & $\mathrm{V}(1)$ \\
\hline Ala 33 & Canada (1) & & \\
\hline Ala 34 & United States (1) & $1 L(1)$ & $\mathrm{G}(1)$ \\
\hline Ala 35 & South Africa (1) & & \\
\hline Alb 36 & United States (1) & $1 \mathrm{~L}(1)$ & $\mathrm{G}(1)$ \\
\hline Alc 37 & Canada (1) & $2 \mathrm{~L}(1)$ & \\
\hline Alc 38 & United States (1) & $1 \mathrm{U}(1)$ & \\
\hline A.lc 39 & Norway (1) & $3 \mathrm{~L}(1)$ & $S(1)$ \\
\hline Alc 40 & France (1) & & \\
\hline Alc 40 & Spain (1) & $3 \mathbf{L}(1)$ & \\
\hline Alc 41 & France (1) & & \\
\hline Alc 42 & Switzerland (1) & $3 \mathrm{~L}(1)$ & \\
\hline Alc 43 & France (1) & & \\
\hline Alc 43 & Switzerland (1) & $11 \mathrm{~L}(1)$ & \\
\hline Alc 44 & United States (1) & $1 \mathrm{~L}(1)$ & \\
\hline Alc 45 & The Gambia (3) & $1 L(1)$ & \\
\hline Alc 46 & Australia (1) & & \\
\hline Alc 47 & Scotland (1) & & \\
\hline Alc 48 & Canada (1) & $26 \mathrm{~L}(1)$ & \\
\hline Ald 49 & United States (1) & $1 \mathrm{H}(1)$ & \\
\hline Ald 50 & Ghana (2) & & $\mathrm{G}(1), \mathrm{V}(1)$ \\
\hline Ald 51 & Scotland (1) & & \\
\hline Ale 52 & Ghana (1) & & \\
\hline Alf 53 & Denmark (1) & & \\
\hline A2a 54 & Canada (1) & $1 \mathrm{~L}(1)$ & \\
\hline A2a 55 & Canada (1) & & \\
\hline A2a 56 & The Gambia (1) & & \\
\hline A2a 56 & United States (1) & & \\
\hline A2a 57 & Hawaii (1) & & \\
\hline A2a 57 & United States (1) & $3 \mathrm{~L}(1)$ & \\
\hline
\end{tabular}


Table 4. (continued)

\begin{tabular}{|c|c|c|c|}
\hline $\begin{array}{l}\text { Group } \\
\text { ET }\end{array}$ & $\begin{array}{c}\text { Geographic source } \\
\text { (no. of isolates) }\end{array}$ & $\begin{array}{c}\text { OMP } \\
\text { pattern* }\end{array}$ & $\begin{array}{c}\text { cap } \\
\text { region } \\
\text { RFLP } \\
\text { pattern }\end{array}$ \\
\hline A2a 58 & Malaysia (5) & $16 \mathrm{~L}(1)$ & $G(1)$ \\
\hline A2a 12.8 & Alaska (3) & $3 L(3)$ & \\
\hline A2a 12.8 & Argentina (1) & $2 \mathbf{L}(1)$ & \\
\hline A2a 12.8 & Australia (2) & & \\
\hline A2a 12.8 & Canada (72) & $3 \mathrm{~L}(6)$ & \\
\hline A2a 12.8 & Denmark (2) & & \\
\hline A2a 12.8 & Dominican Republic (20) & & \\
\hline A2a 12.8 & England (1) & & \\
\hline A2a 12.8 & Finland (44) & $3 \mathrm{~L}(3)$ & \\
\hline A2a 12.8 & France (5) & $2 \mathrm{~L}(1)$ & \\
\hline A2a 12.8 & The Gambia (18) & $2 \mathrm{~L}(6)$ & \\
\hline $\mathrm{A} 2 \mathrm{a} 12.8$ & Ghana (2) & & $S(2)$ \\
\hline A2a 12.8 & Hawaii (4) & & \\
\hline A2a 12.8 & Japan (10) & $3 \mathrm{~L}(3), 16 \mathrm{~L}(1)$ & \\
\hline $\mathrm{A} 2 \mathrm{a} 12.8$ & Malaysia (52) & $\begin{array}{l}3 \mathrm{~L}(10), 18 \mathrm{~L}(3) \\
1 \mathrm{~L}(1), 2 \mathrm{~L}(1) \\
13.1 \mathrm{~L}(1) \\
27 \mathrm{~L}(1)\end{array}$ & $\mathbf{S}(2)$ \\
\hline A2a 12.8 & New Zealand (4) & $3 \mathrm{~L}(4)$ & \\
\hline A2a 12.8 & Norway (5) & & \\
\hline A2a 12.8 & Papua New Guinea (22) & $\begin{array}{l}1 \mathrm{~L}(9), 3 \mathrm{~L}(5) \\
13 \mathrm{~L}(1)\end{array}$ & $S(3)$ \\
\hline A2a 12.8 & Philippines (6) & $\begin{array}{l}1 \mathrm{~L}(3), 3 \mathrm{~L}(2) \\
18 \mathrm{~L}(1)\end{array}$ & \\
\hline A2a 12.8 & South Africa (9) & $2 \mathrm{~L}(2)$ & $S(1)$ \\
\hline A2a 12.8 & Scotland (1) & & \\
\hline A2a 12.8 & South Korea (2) & & \\
\hline A2a 12.8 & Sweden (14) & & \\
\hline A2a 12.8 & Switzerland (9) & & \\
\hline A2a 12.8 & Thailand (7) & $\begin{array}{l}2 \mathrm{~L}(3), 3 \mathrm{~L}(4) \\
1 \mathrm{~L}(1)\end{array}$ & $S(1)$ \\
\hline A2a 12.8 & United States (76) & $\begin{array}{l}3 \mathrm{~L}(20), 13 \mathrm{~L}(6) \\
14 \mathrm{~L}(1)\end{array}$ & $S(2)$ \\
\hline A2a 12.8 & Uruguay (1) & $2 \mathrm{~L}(1)$ & \\
\hline A2a 59 & Malaysia (1) & $3 \mathrm{~L}(1)$ & \\
\hline A2a 59 & Thailand (1) & $1 \mathrm{~L}(1)$ & \\
\hline A2a 60 & Denmark (1) & & \\
\hline A2a 60 & Kenya (4) & $3 \mathrm{~L}(1)$ & $S(4)$ \\
\hline A2a 60 & Malaysia (4) & $3 L(3)$ & \\
\hline A2a 60 & South Africa (1) & & \\
\hline A2a 60 & Sweden (1) & & \\
\hline A2a 61 & Denmark (1) & & \\
\hline A2a 62 & United States (1) & $3 \mathrm{~L}(1)$ & \\
\hline A2a 63 . & Norway (1) & $3 \mathrm{~L}(1)$ & \\
\hline A2a 64 & United States (1) & $3 L(1)$ & $S(1)$ \\
\hline A2a 65 & Canada (1) & & \\
\hline A2a 66 & United States (1) & $11 L(1)$ & $S(1)$ \\
\hline A2a 67 & Denmark (1) & & \\
\hline A2a 67 & Finland (1) & & \\
\hline A2a 67 & Norway (1) & $3 L(1)$ & \\
\hline A2a 68 & Norway (1) & & \\
\hline A2a 68 & South Africa (1) & & \\
\hline A2a 69 & Malaysia (4) & & \\
\hline A2a 70 & Papua New Guinea (2) & & \\
\hline
\end{tabular}

Table 4. (continued)

\begin{tabular}{|c|c|c|c|}
\hline $\begin{array}{l}\text { Group } \\
\text { ET }\end{array}$ & $\begin{array}{l}\text { Geographic source } \\
\text { (no. of isolates) }\end{array}$ & $\begin{array}{c}\text { OMP } \\
\text { pattern* }\end{array}$ & $\begin{array}{c}\text { cap } \\
\text { region } \\
\text { RFLP } \\
\text { pattern }\end{array}$ \\
\hline A2a 71 & England (1) & & $S(1)$ \\
\hline A2a 72 & Iceland (1) & $3 L(1)$ & \\
\hline A2a 73 & Canada (1) & & \\
\hline A2a 74 & South Africa (1) & $2 L(1)$ & \\
\hline A2a 12.6 & Australia (1) & & \\
\hline A2a 12.6 & Denmark (1) & & \\
\hline A2a 12.6 & France (3) & & \\
\hline A2a 12.6 & Philippines (1) & $18 \mathrm{~L}(1)$ & \\
\hline A2a 12.6 & Switzerland (1) & & \\
\hline A2a 12.6 & United States (4) & $14 \mathrm{~L}(1), 18 \mathrm{~L}(1)$ & $S(1)$ \\
\hline A2a 12.10 & Hawaii (1) & $11 \mathrm{~L}(1)$ & \\
\hline $\mathrm{A} 2 \mathrm{a} 12.9$ & Canada (1) & & \\
\hline A2a 12.9 & Dominican Republic (1) & & \\
\hline A2a 12.9 & Finland (3) & & \\
\hline A2a 12.9 & France (1) & & \\
\hline A2a 12.9 & Japan (2) & $16 \mathrm{~L}(2)$ & \\
\hline A2a 12.9 & Netherlands (1) & & \\
\hline $\mathrm{A} 2 \mathrm{a} 12.9$ & Norway (2) & & \\
\hline A2a 12.9 & South Korea (5) & & \\
\hline A2a 12.9 & Sweden (5) & & $\mathbf{S}(1)$ \\
\hline A2a 12.9 & Switzerland (2) & & \\
\hline A2a 75 & Denmark (1) & $3 \mathrm{~L}(1)$ & \\
\hline A2a 12.11 & Philippines (1) & $29 L(1)$ & \\
\hline A2a 76 & South Africa (1) & & \\
\hline A2a 12.7 & Alaska (14) & $3 L(14)$ & \\
\hline A2a 12.7 & Australia (1) & & \\
\hline $\mathrm{A} 2 \mathrm{a} 12.7$ & Canada (23) & $31 L(1)$ & $S(1)$ \\
\hline A2a 12.7 & Denmark (1) & $3 \mathrm{~L}(1)$ & \\
\hline A2a 12.7 & England (1) & & \\
\hline A2a 12.7 & Hawaii (2) & & \\
\hline A2a 12.7 & Malaysia (1) & & \\
\hline A2a 12.7 & Norway (2) & & \\
\hline A2a 12.7 & Switzerland (4) & & \\
\hline A2a 12.7 & United States (27) & $3 L(9), 13 L(3)$ & \\
\hline A2a 77 & Switzerland (2) & $3 \mathrm{~L}(1)$ & \\
\hline A2a 78 & United States (2) & $1 \mathrm{~L}(1) \dagger, 16 \mathrm{~L}(1)$ & $S(1)$ \\
\hline A2a 79 & Switzerland (1) & $3 \mathrm{~L}(1)$ & \\
\hline $\mathrm{A} 2 \mathrm{a} 12.5$ & Alaska (2) & $3 \mathrm{~L}(2)$ & \\
\hline $\mathrm{A} 2 \mathrm{a} 12.5$ & Argentina (3) & $3 \mathrm{~L}(1)$ & \\
\hline $\mathrm{A} 2 \mathrm{a} 12.5$ & Australia (24) & & \\
\hline A2a 12.5 & Canada (63) & $3 \mathrm{~L}(7)$ & \\
\hline A 2 a 12.5 & Denmark (22) & $3 \mathrm{~L}(2)$ & \\
\hline A2a 12.5 & Dominican Republic (18) & & \\
\hline $\mathrm{A} 2 \mathrm{a} 12.5$ & England (12) & & \\
\hline A2a 12.5 & Finland (23) & $3 L(2)$ & \\
\hline A2a 12.5 & France $(60)$ & $3 \mathrm{~L}(6)$ & \\
\hline A2a 12.5 & Greenland (3) & & \\
\hline A2a 12.5 & Hawaii (2) & & \\
\hline A2a 12.5 & Iceland (3) & $3 \mathrm{~L}(3)$ & $S(1)$ \\
\hline A2a 12.5 & New Zealand (11) & $3 \mathrm{~L}(11)$ & \\
\hline A2a 12.5 & Netherlands (5) & $3 \mathrm{~L}(3)$ & \\
\hline $\mathrm{A} 2 \mathrm{a} 12.5$ & Norway (16) & $3 L(3)$ & \\
\hline $\mathrm{A} 2 \mathrm{a} 12.5$ & Papua New Guinea (2) & $3 \mathrm{~L}(2)$ & \\
\hline $\mathrm{A} 2 \mathrm{a} 12.5$ & South Africa (3) & & \\
\hline
\end{tabular}


Table 4. (continued)

\begin{tabular}{|c|c|c|c|}
\hline $\begin{array}{l}\text { Group } \\
\text { ET }\end{array}$ & $\begin{array}{c}\text { Geographic source } \\
\text { (no. of isolates) }\end{array}$ & $\begin{array}{c}\text { OMP } \\
\text { pattern* }\end{array}$ & $\begin{array}{c}\text { cap } \\
\text { region } \\
\text { RFLP } \\
\text { pattern } \\
\end{array}$ \\
\hline $\mathrm{A} 2 \mathrm{a} 12.5$ & Scotland (13) & & \\
\hline $\mathrm{A} 2 \mathrm{a} 12.5$ & Spain (69) & & \\
\hline A2a 12.5 & Sweden (34) & & \\
\hline A2a 12.5 & Switzerland (88) & $3 L(10)$ & \\
\hline $\mathrm{A} 2 \mathrm{a} 12.5$ & United States (21) & $3 L(8)$ & $\mathbf{S}(1)$ \\
\hline A $2 a 80$ & Denmark (1) & & \\
\hline A2a 80 & United States (1) & & \\
\hline A2a 81 & South Africa (1) & $3 L(1)$ & \\
\hline A2a 82 & France (2) & & \\
\hline A2a 82 & Sweden $(6)$ & & \\
\hline A2a 82 & United States (1) & $3 \mathrm{~L}(1)$ & \\
\hline A2a 83 & Australia (1) & & \\
\hline A2a 84 & Canada (1) & & \\
\hline A2a 85 & Canada (1) & $3 \mathrm{~L}(1)$ & \\
\hline A2a 21.8 & Alaska (1) & $1 \mathbf{L}(1)^{\dagger}$ & \\
\hline A2a 21.8 & Dominican Republic (1) & & \\
\hline A2a 21.8 & Finland (19) & $1 \mathrm{~L}(3)$ & \\
\hline A2a 21.8 & Iceland (1) & $1 \mathrm{~L}(1)$ & \\
\hline $\mathrm{A} 2 \mathrm{a} 21.8$ & Malaysia (6) & $1 \mathbf{L}(1)$ & \\
\hline $\mathrm{A} 2 \mathrm{a} 21.8$ & Sweden (4) & & \\
\hline A2a 21.8 & United States (1) & $1 \mathrm{~L}(1)$ & \\
\hline A2a 21.9 & Sweden (1) & & $S(1)$ \\
\hline A2a 21.5 & Australia (3) & & \\
\hline A2a 86 & United States (1) & $3 L(1)$ & \\
\hline A2a 87 & Finland (1) & & \\
\hline A2a 88 & Finland (2) & & \\
\hline A2a 89 & France (1) & & \\
\hline A2a 12.0 & Alaska (1) & $3 \mathrm{~L}(1)$ & \\
\hline $\mathrm{A} 2 \mathrm{a} 12.0$ & Australia (2) & & \\
\hline A2a 12.0 & Canada (4) & $3 L(1)$ & $S(1)$ \\
\hline A2a 12.0 & Denmark (1) & & \\
\hline A2a 12.0 & Finland (1) & & \\
\hline A2a 12.0 & New Zealand (1) & $3 \mathrm{~L}(1)$ & \\
\hline A2a 12.0 & Switzerland (1) & & \\
\hline A2a 90 & Canada (1) & & \\
\hline A2a 90 & Sweden (1) & & \\
\hline A2a 91 & Canada (1) & $3 \mathrm{~L}(1)$ & \\
\hline A2a 92 & United States (1) & & $S(1)$ \\
\hline A2a 93 & Canada (1) & & \\
\hline A2a 93 & Dominican Republic (1) & & \\
\hline A2a 93 & Finland (1) & & \\
\hline A2a 93 & Scotland (2) & & \\
\hline A2a 93 & Spain (1) & & \\
\hline A2a 93 & Sweden (2) & & \\
\hline A2a 94 & United States (1) & & \\
\hline A2a 95 & United States (2) & & \\
\hline A2a 96 & Denmark (1) & & \\
\hline A2a 96 & Greenland (1) & & \\
\hline A2a 97 & Canada (1) & & \\
\hline A2a 98 & United States (1) & & \\
\hline A2a 99 & Malaysia (2) & $3 \mathrm{~L}(1)$ & \\
\hline A2a 100 & Canada (17) & $3 \mathrm{~L}(3)$ & $S(1)$ \\
\hline A2a 100 & Finland (2) & & \\
\hline A2a 101 & United States (1) & $14.1 \mathrm{~L}(1)$ & $S(1)$ \\
\hline
\end{tabular}

Table 4. (continued)

\begin{tabular}{|c|c|c|c|}
\hline $\begin{array}{l}\text { Group } \\
\text { ET }\end{array}$ & $\begin{array}{c}\text { Geographic source } \\
\text { (no. of isolates) }\end{array}$ & $\begin{array}{c}\text { OMP } \\
\text { pattern* }\end{array}$ & $\begin{array}{c}\text { cap } \\
\text { region } \\
\text { RFLP } \\
\text { pattern }\end{array}$ \\
\hline A2a 102 & United States (1) & $22 \mathrm{~L}(1)$ & $S(1)$ \\
\hline A2a 103 & Alaska (7) & $\begin{array}{c}5 \mathrm{~L}(6), 3 \mathrm{~L}(1) \\
5.1 \mathrm{~L}(1)\end{array}$ & $S(1)$ \\
\hline A2a 103 & United States (1) & & \\
\hline A2a 104 & Switzerland (2) & $3 \mathrm{~L}(1)$ & \\
\hline A2a 105 & Canada (1) & & \\
\hline A2a 105 & Dominican Republic (1) & & \\
\hline $\mathrm{A} 2 \mathrm{a} 105$ & South Africa (2) & & \\
\hline $\mathrm{A} 2 \mathrm{a} 105$ & Switzerland (2) & & \\
\hline A2a 106 & Alaska (1) & $13 \mathrm{~L}(1)$ & \\
\hline A2a 107 & Canada (1) & & \\
\hline $\mathrm{A} 2 \mathrm{a} 108$ & Switzerland (1) & $3 L(1)$ & \\
\hline $\mathrm{A} 2 \mathrm{a} 108$ & United States (1) & & \\
\hline A2a 109 & South Africa (1) & $2 \mathrm{~L}(1)$ & \\
\hline A2a 110 & Denmark (1) & $1 \mathrm{~L}(1)$ & \\
\hline A2a 110 & Finland (1) & & \\
\hline A2a 111 & Canada (1) & & \\
\hline A2b 112 & Canada (1) & & \\
\hline $\mathrm{A} 2 \mathrm{~b} 113$ & Canada (1) & & \\
\hline A2b 114 & Alaska (1) & $5 \mathrm{~L}(1)$ & \\
\hline A2c 115 & South Africa (1) & $2 \mathrm{~L}(\mathrm{l})$ & \\
\hline A2d 116 & Dominican Republic (1) & $16 \mathrm{~L}(1)$ & \\
\hline A2d 117 & Dominican Republic (1) & $16 L(1)$ & \\
\hline A2d 118 & United States (1) & $5 \mathrm{~L}(1)$ & $S(1)$ \\
\hline A2e 21.6 & Alaska (12) & $13 \mathrm{~L}(12)$ & \\
\hline A2e 21.6 & Canada (2) & & \\
\hline A2e 21.6 & Denmark (1) & & \\
\hline $\mathrm{A} 2 \mathrm{e} 21.6$ & Guatemala (1) & & \\
\hline A2e 21.6 & New Zealand (1) & $13 \mathrm{~L}(1)$ & \\
\hline A2e 21.6 & Papua New Guinea (1) & $13 L(1)$ & \\
\hline A2e 21.6 & Philippines (1) & & \\
\hline A2e 21.6 & Thailand (1) & $13 \mathrm{~L}(1)$ & \\
\hline A2e 21.6 & United States (37) & $\begin{array}{l}13 \mathrm{~L}(21), 11 \mathrm{~L}(1), \\
3 \mathrm{~L}(1) \dagger\end{array}$ & $S(8)$ \\
\hline A2e 119 & United States (1) & $11 \mathrm{~L}(1)$ & \\
\hline A2e 120 & Alaska (1) & $13 \mathrm{~L}(1)$ & \\
\hline A2e 121 & Canada (1) & & \\
\hline A2e 121 & Hawaii (1) & $13 L(1)$ & \\
\hline A2e 122 & United States (2) & & $S(2)$ \\
\hline A2e 123 & United States (1) & & \\
\hline A2e 124 & Alaska (1) & $13 \mathrm{~L}(1)$ & \\
\hline A2e 125 & United States (1) & & \\
\hline A2e 126 & Denmark (1) & & \\
\hline A2f 127 & Denmark (1) & & \\
\hline A3 128 & Hawaii (1) & & \\
\hline A4 129 & Canada (1) & & \\
\hline A5 130 & South Africa (1) & $1 \mathrm{~L}(1)$ & \\
\hline B1a 131 & United States (1) & & \\
\hline B1a 132 & Malaysia (1) & $6 \mathrm{U}(1)$ & \\
\hline
\end{tabular}


Table 4. (continued)

\begin{tabular}{|c|c|c|c|}
\hline $\begin{array}{l}\text { Group } \\
\text { ET }\end{array}$ & $\begin{array}{l}\text { Geographic source } \\
\text { (no. of isolates) }\end{array}$ & $\begin{array}{c}\text { OMP } \\
\text { pattern* }\end{array}$ & $\begin{array}{c}\text { cap } \\
\text { region } \\
\text { RFLP } \\
\text { pattern }\end{array}$ \\
\hline B1b 25.6 & Canada (6) & $6 \mathrm{U}(1)$ & $S(2)$ \\
\hline B1b 25.6 & Malaysia (4) & $6 \mathrm{U}(2)$ & \\
\hline B1b 25.6 & Papua New Guinea (26) & $6 \mathrm{U}(4)$ & $S(1)$ \\
\hline Blb 25.6 & Thailand (1) & $6 \mathrm{U}(1)$ & \\
\hline B1b 25.6 & United States (10) & $6 \mathrm{U}(9)$ & $\$(3)$ \\
\hline B1b 133 & United States (1) & & $S(1)$ \\
\hline Blb 134 & Canada (1) & & \\
\hline B1b 135 & Papua New Guinea (2) & $6 U(2)$ & \\
\hline Blb 136 & Hawaii (1) & & \\
\hline Blb 137 & Denmark (2) & & \\
\hline B1b 137 & France (1) & & \\
\hline Blb 138 & Netherlands (1) & $6 \mathrm{U}(1)$ & $\mathbf{S}(1)$ \\
\hline Blb 138 & Papua New Guinea (1) & $6 \mathrm{U}(1)$ & \\
\hline Blb 139 & Kenya (1) & $6 \mathrm{U}(1)$ & \\
\hline B1b 139 & Malaysia (1) & & $S(1)$ \\
\hline Blb 139 & Sweden (1) & & \\
\hline Blb 140 & Kenya (1) & & $S(1)$ \\
\hline Blb 141 & Canada (2) & & \\
\hline Blb 141 & The Gambia (5) & $6 \mathrm{U}(3)$ & \\
\hline Blb 141 & South Africa (4) & & \\
\hline Blb 141 & Sweden (2) & & \\
\hline B1b 142 & Hawaii (1) & & \\
\hline Blb 143 & United States (1) & $23 \mathrm{U}(1)$ & $S(1)$ \\
\hline Blb 144 & The Gambia (1) & & \\
\hline B1b 145 & Alaska (1) & $6 \mathrm{U}(1)$ & \\
\hline B1b 145 & Canada (1) & & \\
\hline Blb 145 & Dominican Republic (1) & & \\
\hline Blb 145 & New Zealand (2) & $6 \mathrm{U}(1), 12 \mathrm{U}(1)$ & $\mathbf{S}(1)$ \\
\hline Blb 145 & Papua New Guinea (3) & & $\mathbf{S}(\mathbf{1})$ \\
\hline B1b 145 & United States (1) & $24 \mathrm{U}(1)$ & $\mathbf{S}(1)$ \\
\hline B1b 146 & United States (1) & & \\
\hline B1b 147 & Dominican Republic (6) & & \\
\hline B1b 148 & Dominican Republic (1) & & \\
\hline B1b 149 & Dominican Republic (1) & $6 U(1)$ & \\
\hline B1b 150 & South Africa (2) & $6 \mathrm{U}(1)$ & \\
\hline BIb 150 & Switzerland (2) & & \\
\hline Blb 151 & Switzerland (2) & $6 \mathrm{U}(1)$ & $S(1)$ \\
\hline Blb 152 & South Africa (12) & $6 \mathrm{U}(2)$ & $S(1)$ \\
\hline B1b 153 & South Africa (1) & & \\
\hline B1c 154 & Dominican Republic (1) & & \\
\hline B1d 155 & Alaska (1) & $1 \mathrm{U}(1)$ & \\
\hline Ble 156 & South Korea (1) & & \\
\hline B1f 157 & England (11) & & $\mathrm{d}(9)$ \\
\hline BIf 158 & United States (1) & & $\mathrm{d}(1)$ \\
\hline Blf 159 & England (4) & & $\mathrm{d}(2)$ \\
\hline B1f 160 & United States (1) & & \\
\hline B1f 161 & Papua New Guinea (1) & & $d(1)$ \\
\hline Blg 162 & Malaysia (8) & & $\mathrm{d}(8)$ \\
\hline Blg 163 & Kenya (1) & & $\mathrm{d}(1)$ \\
\hline B2a 164 & United States (1) & $\mathrm{a} 1 \mathrm{U}(1)$ & $\mathrm{aT}(1)$ \\
\hline B2b 165 & Papua New Guinea (2) & $\operatorname{alU}(2)$ & $\mathrm{aT}(2)$ \\
\hline
\end{tabular}

Table 4. (continued)

\begin{tabular}{|c|c|c|c|}
\hline $\begin{array}{l}\text { Group } \\
\text { ET }\end{array}$ & $\begin{array}{c}\text { Geographic source } \\
\text { (no. of isolates) }\end{array}$ & $\begin{array}{c}\text { OMP } \\
\text { pattern* }\end{array}$ & $\begin{array}{c}\text { cap } \\
\text { region } \\
\text { RFLP } \\
\text { pattern }\end{array}$ \\
\hline B2b 166 & Papua New Guinea (1) & alU(1) & $\operatorname{aT}(1)$ \\
\hline B2b 167 & The Gambia (2) & alU(2) & $\mathbf{a T}(2)$ \\
\hline B2b 167 & Malaysia (7) & alU(7) & $\mathbf{a T}(7)$ \\
\hline B2b 168 & Malaysia (1) & alU(1) & $\mathbf{a T}(1)$ \\
\hline B2c 169 & Malaysia (2) & $\mathrm{a} 1 \mathrm{U}(2)$ & $\mathbf{a T}(2)$ \\
\hline B2d 170 & Kenya (1) & alU(1) & aN(1) \\
\hline B3 171 & The Gambia (2) & & \\
\hline B4 172 & Japan (sero b) (1) & & \\
\hline B4 173 & The Gambia (sero a) (3) & a5L(3) & aN(3) \\
\hline B4 173 & Malaysia (sero a) (1) & alU(1) & $\mathrm{aT}(1)$ \\
\hline B4 174 & Kenya (sero a) (1) & asL(1) & aN(1) \\
\hline B5 175 & Iceland (1) & & \\
\hline C1 176 & Switzerland (1) & & \\
\hline D1a 177 & United States (1) & & $\operatorname{cl}(1)$ \\
\hline D1a 178 & Malaysia (1) & & $\operatorname{cl}(1)$ \\
\hline D1b 179 & Unknown (1) & & \\
\hline D1b 180 & Malaysia (3) & & $\operatorname{cl}(3)$ \\
\hline D1b 181 & England (1) & & $\operatorname{cl}(1)$ \\
\hline D2 182 & Unknown (1) & & $\operatorname{cl}(1)$ \\
\hline D2 183 & England (1) & & $\operatorname{cl}(1)$ \\
\hline D3 184 & United States (1) & & $\operatorname{cl}(1)$ \\
\hline D4 185 & England (1) & & $\mathrm{c} 2(1)$ \\
\hline D5 186 & Kenya (1) & & $c 2(1)$ \\
\hline E1 187 & Kenya (1) & & \\
\hline F1 188 & England (1) & & e(1) \\
\hline F1 189 & England (5) & & $e(2)$ \\
\hline F1 190 & England (2) & & $e(2)$ \\
\hline F2a 191 & England (2) & & \\
\hline F2a 191 & United States (3) & & e(1) \\
\hline F2a 191 & Unknown (1) & & \\
\hline F2a 192 & United States (1) & & \\
\hline F2a 193 & England (3) & & $e(1)$ \\
\hline F2a 194 & Malaysia (1) & & e(1) \\
\hline F2a 195 & Unknown (3) & & \\
\hline F2a 196 & England (2) & & e(1) \\
\hline F2a 197 & England (4) & & \\
\hline F2a 198 & England (1) & & e(1) \\
\hline F2a 199 & Unknown (1) & & $e(1)$ \\
\hline F2a 200 & Unknown (1) & & e(1) \\
\hline F2a 201 & England (1) & & $e(1)$ \\
\hline F2b 202 & England (1) & & Anom $\ddagger$ \\
\hline F2b 203 & Papua New Guinea (1) & & $\mathbf{e}(1)$ \\
\hline F2c 204 & England (31) & & $e(3)$ \\
\hline F2c 205 & Kenya (2) & & \\
\hline F2c 206 & Malaysia (1) & & $e(1)$ \\
\hline F2c 207 & England (1) & & $e(1)$ \\
\hline F2c 208 & England (2) & & $\mathbf{e}(1)$ \\
\hline
\end{tabular}


Table 4. (continued)

\begin{tabular}{|c|c|c|c|}
\hline $\begin{array}{l}\text { Group } \\
\text { ET }\end{array}$ & $\begin{array}{c}\text { Geographic source } \\
\text { (no. of isolates) }\end{array}$ & $\begin{array}{c}\text { OMP } \\
\text { pattern* }\end{array}$ & $\begin{array}{c}c a p \\
\text { region } \\
\text { RFLP } \\
\text { pattern }\end{array}$ \\
\hline F2c 209 & England (1) & & \\
\hline F/c 210 & England (2) & & $e(1)$ \\
\hline F2c 211 & England (1) & & $e(1)$ \\
\hline F2c 212 & England (1) & & \\
\hline F2c 213 & Malaysia (1) & & \\
\hline F2c 214 & England (2) & & $e(2)$ \\
\hline F2c 215 & England (5) & & $e(1)$ \\
\hline F2c 216 & England (6) & & $e(3)$ \\
\hline F2c 217 & England (1) & & $e(1)$ \\
\hline G1 218 & Kenya (1) & & $e(1)$ \\
\hline H1a 219 & England (13) & a2H(10), a7H(3) & $\mathrm{aM}(13)$ \\
\hline H1a 219 & Unknown (1) & $\mathrm{a} 2 \mathrm{H}(1)$ & $\mathrm{aM}(1)$ \\
\hline H1a 220 & England (1) & $\mathrm{a} 4 \mathrm{H}(1)$ & $\mathrm{aM}(1)$ \\
\hline H1a 221 & England (1) & $\mathrm{a} 2 \mathrm{H}(1)$ & $\mathrm{aM}(1)$ \\
\hline H1b 222 & England (1) & $\mathrm{a} 6 \mathrm{H}(1)$ & $\mathrm{aM}(1)$ \\
\hline H1b 223 & England (1) & $\mathrm{a} 6 \mathrm{H}(1)$ & $\operatorname{aM}(1)$ \\
\hline H1b 224 & England (3) & a6H(3) & $\operatorname{aM}(3)$ \\
\hline H1b 225 & England (1) & $\mathrm{a} 6 \mathrm{H}(1)$ & $\mathbf{a M}(1)$ \\
\hline H1b 226 & England (1) & $\mathrm{a} 6 \mathrm{H}(1)$ & $\mathbf{a M}(1)$ \\
\hline I1a 227 & England (2) & $\mathrm{a} 4 \mathrm{H}(2)$ & $\mathrm{aM}(2)$ \\
\hline Ila 228 & England (1) & $\mathrm{a} 4 \mathrm{H}(1)$ & $\mathrm{aM}(1)$ \\
\hline Ila 229 & England (3) & $\mathrm{a} 4 \mathrm{H}(2), \mathrm{a} 8 \mathrm{H}(1)$ & $\mathrm{aM}(3)$ \\
\hline I1b 230 & Dominican Republic (1) & a5H(1) & $\mathrm{aM}(1)$ \\
\hline J1a 231 & United States (1) & & \\
\hline J1a 232 & United States (1) & & $O(1)$ \\
\hline J1b 233 & United States (1) & $8-H^{\S}(1)$ & \\
\hline J1b 234 & Alaska (1) & $8 \mathbf{H}(1)$ & \\
\hline J1b 235 & Alaska (2) & $8 \mathrm{H}(2)$ & $O(1)$ \\
\hline J1b 235 & Norway (1) & $30 \mathrm{H}(1)$ & \\
\hline J1b 236 & Canada (1) & $8 \mathbf{H}(1)$ & \\
\hline J1b 236 & United States (1) & $8 \mathrm{H}(1)$ & \\
\hline J1b 237 & United States (1) & $8 \mathrm{H}(1)$ & \\
\hline J2 238 & South Africa (1) & $25 \mathrm{~L}(1)$ & \\
\hline J3239 & United States (1) & $17 \mathrm{H}(1)$ & \\
\hline Kla 240 & United States (1) & & \\
\hline K1b 241 & England (2) & & $O(1)$ \\
\hline K1c 242 & England (1) & & $F(1)$ \\
\hline K1c 243 & England (1) & & \\
\hline K1c 244 & Malaysia (1) & & $F(1)$ \\
\hline K1c 245 & Malaysia (1) & & \\
\hline K1c 246 & Malaysia (1) & & \\
\hline K1c 247 & Malaysia (1) & & \\
\hline K1c 248 & Malaysia (1) & & \\
\hline K1c 249 & Malaysia (2) & & $F(1)$ \\
\hline K1C 250 & Malaysia (1) & & $F(1)$ \\
\hline K1c 251 & Malaysia (2) & & $\mathbf{F}(1)$ \\
\hline K1c 252 & Malaysia (1) & & \\
\hline K1c 253 & Malaysia (1) & & $F(1)$ \\
\hline K1c 254 & Malaysia (1) & & $F(1)$ \\
\hline
\end{tabular}

Table 4. (continued)

\begin{tabular}{llcl} 
Group & $\begin{array}{c}\text { Geographic source } \\
\text { (no. of isolates) }\end{array}$ & $\begin{array}{c}\text { OMP } \\
\text { pattern* }\end{array}$ & $\begin{array}{c}\text { cap } \\
\text { region } \\
\text { RFLP } \\
\text { pattern }\end{array}$ \\
\hline K1d 255 & United States (1) & \\
K2 256 & United States (1) & $\mathrm{O}(1)$ \\
K2 256 & Unknown (1) & \\
K2 257 & England (1) & \\
K2 258 & England (1) & Un*(1) \\
K2 259 & England (1) & $\mathrm{O}(1)$ \\
K2 260 & England (7) & $\mathrm{O}(1), \mathrm{U}{ }^{*}(1)$ \\
K2 261 & England (1) & $\mathrm{O}(1)$ \\
K2 262 & England (2) & $\mathrm{O}(1)$ \\
K2 262 & Unknown (2) & $\mathrm{O}(2)$ \\
K2 263 & England (3) & $\mathrm{O}(1)$ \\
K2 264 & England (1) & $\mathrm{Un}{ }^{*}(1)$ \\
K2 265 & England (5) & $\mathrm{O}(3)$ \\
K2 266 & England (4) & \\
L1 267 & Kenya (1) & \\
\hline
\end{tabular}

* For most geographic source groups, strains were randomly selected for OMP subtype analysis. The OMP subtype designations for serotype $b$ isolates are those reported by the Granoff laboratory $[8,19]$, and serotype a OMP patterns have been distinguished by the Moxon laboratory [25]. To avoid confusion, serotype a pattern designations are preceded by the letter "a." Note that serotype a and serotype $b$ strains with the same OMP type designation may actually not be identical in OMP electrophoretic pattern. The cap region RFLP patterns have been reported previously $[24,26]$. The number of isolates identified of each OMP pattern or cap region RFLP pattern is given in parentheses.

$\dagger$ Pattern similar to, but not identical with, designated OMP subtype.

$\ddagger$ Anomalous.

$\$$ Similar to type 8 but apparently lacking the P1 band.

"Unclassified.

types account for $70 \%$ of all serotype b strains in the sample, and, moreover, $81 \%$ of all type $b$ strains examined belong to only nine of the 280 multilocus enzyme genotypes identified (table 4).

\section{cap Region RFLP Patterns and Genotypic Diversity} in cap Region RFLP Pattern

The cap region is a 20 - to 40 -kilobase $(\mathrm{kb})$ segment of the chromosome containing genes necessary for the production of capsule polysaccharide $[33,36$, 37]. Previously 14 cap region RFLP patterns were identified among 222 isolates of six serotypes examined [21]. The approximate sizes (in kilobases) of the DNA fragments characterizing each pattern are indicated in figure 2; their distributions among the major branches of the dendrogram are shown in figure 
1 and described in detail elsewhere [21]. All RFLP patterns were serotype specific.

For both serotypes a and $b$, the cap region patterns of isolates in the two primary phylogenetic divisions were very different. The $a(T)$ and $a(N)$ patterns of serotype a strains in division I have three of four fragments of common size, but fragments of similar size do not occur in the a(M) pattern of serotype a strains in division II. Serotype b strains in division $I$ had patterns $b(S), b(V)$, and $b(G)$, which share most of their fragments, whereas serotype $b$ strains in division II had the $b(O)$ pattern, which has only two of its seven fragments ( 2.1 and $2.7 \mathrm{~kb}$, which are type $b$ specific) in common with the other serotype $b$ patterns.

Each of the 14 cap region RFLP patterns occurred in isolates of several multilocus genotypes, with as many as 30 ETs being identified among the 53 isolates with pattern $b(S)$. For patterns $b(S), b(O), d$, and $e$, mean genetic diversity $(H)$ among ETs was roughly equivalent to that recorded for ETs of all isolates of the same serotype, whereas among ETs of the other probe patterns, $H$ was, on average, equal to only $64 \%$ of that of ETs of the same serotype (table 5) [21]. These results reflect the circumstance that cap patterns are found in isolates that are nonrandom samples of clones of each serotype.

\section{Genotypic Diversity Within OMP Patterns of Serotype b Strains}

The OMP electrophoretic patterns of 587 serotype $b$ isolates were determined and assigned to 33 categories. These include 25 patterns previously described $[19,20]$ and eight newly identified subtypes, as follows: 13.1L, which is similar to but distinguishable from 13L, and $25 \mathrm{~L}, 26 \mathrm{~L}, 27 \mathrm{~L}, 29 \mathrm{~L}, 31 \mathrm{~L}, 30 \mathrm{H}$, and 12U. Most OMP patterns were confined to somewhat closely related clones, with an average of $37 \%$ of the total genetic diversity and $54 \%$ of serotype $b$ genetic diversity being apportioned among ETs represented by isolates of the nine more common OMP subtypes (table 6).

OMP types in cluster $\mathrm{Al}$ were $1 \mathrm{H}$ (80 isolates), 2H (11 isolates), 1L (33 isolates), 2L (115 isolates), $3 \mathrm{~L}$ (three isolates), $11 \mathrm{~L}$ (two isolates), and $4 \mathrm{H}, 19 \mathrm{H}$, $9 \mathrm{~L}, 15 \mathrm{~L}, 18 \mathrm{~L}, 26 \mathrm{~L}$, and $1 \mathrm{U}$, each of which was recorded in a single isolate. Cluster A2 contained OMP types $1 \mathrm{~L}$ (26 isolates), 2L (16 isolates), 3L (172 isolates), $5 \mathrm{~L}$ (eight isolates), $11 \mathrm{~L}$ (four isolates), $13 \mathrm{~L}$ (51 isolates), 14L (two isolates), 16L (six isolates),
Table 5. Mean genetic diversity $(H)$ among ETs within cap locus RFLP patterns of $H$. influenzae.

\begin{tabular}{lrcrc}
\hline Pattern & $\begin{array}{c}\text { No. of } \\
\text { isolates }\end{array}$ & $\begin{array}{c}\text { Mean no. of } \\
\text { alleles }\end{array}$ & $\begin{array}{r}\text { No. of } \\
\text { ETs }\end{array}$ & $\boldsymbol{H}_{\text {ETs }}$ \\
\hline $\mathrm{a}(\mathrm{T})$ & 17 & 1.77 & 7 & 0.235 \\
$\mathrm{a}(\mathrm{N})$ & 5 & 1.29 & 3 & 0.196 \\
$\mathrm{a}(\mathrm{M})$ & 30 & 1.94 & 12 & 0.306 \\
$\mathrm{~b}(\mathrm{G})$ & 14 & 1.88 & 10 & 0.261 \\
$\mathrm{~b}(\mathrm{~V})$ & 14 & 1.53 & 6 & 0.208 \\
$\mathrm{~b}(\mathrm{~S})$ & 53 & 2.65 & 30 & 0.314 \\
$\mathrm{~b}(0)$ & 4 & 1.59 & 3 & 0.373 \\
$\mathrm{c}(\mathrm{l})$ & 9 & 1.82 & 7 & 0.283 \\
$\mathrm{c}(2)$ & 2 & 1.29 & 2 & 0.294 \\
$\mathrm{~d}$ & 22 & 1.41 & 6 & 0.161 \\
$\mathrm{e}$ & 30 & 2.65 & 23 & 0.257 \\
f(F) & 7 & 1.47 & 7 & 0.148 \\
f(0) & 12 & 1.53 & 8 & 0.183 \\
f(un) $\dagger$ & 3 & 1.29 & 3 & 0.176 \\
\multicolumn{1}{c}{ Total } & 222 & 1.72 & 124 & 0.467 \\
\hline
\end{tabular}

* One isolate with a slightly anomalous hybridization pattern was not included in the sample.

$\dagger$ un $=$ unclassified.

18L (six isolates), and one isolate each of 5.1L, 13.1L, 14.1L, 22L, 27L, 29L, and 31L. All 6U isolates were in cluster $\mathrm{B1}$, and $8 \mathrm{H}$ isolates were confined to cluster $\mathbf{J 1}$.

Mean genetic diversity per locus among ETs synthesizing the $\mathrm{H}, \mathrm{L}$, or $\mathrm{U}$ outer membrane protein $\mathrm{Pl}$ was, on average, $81 \%, 50 \%$, and $47 \%$, respectively, of that in the total sample of encapsulated strains and $\sim 60 \%$ of that recorded for serotype $b$ strains

Table 6. Genetic diversity in relation to OMP pattern and P1 type in serotype b $H$. influenzae.

\begin{tabular}{lrrrr}
\hline OMP & $\begin{array}{r}\text { No. of } \\
\text { isolates }\end{array}$ & $\begin{array}{c}\text { No. of } \\
\text { ETs }\end{array}$ & $H_{\text {ETs }}$ & $\begin{array}{c}\text { Mean } \\
\text { no. of } \\
\text { alleles }\end{array}$ \\
\hline IH & 80 & 10 & 0.122 & 1.6 \\
IL & 59 & 19 & 0.223 & 2.2 \\
2L & 117 & 14 & 0.205 & 1.9 \\
3L & 180 & 30 & 0.162 & 2.8 \\
11L & 6 & 6 & 0.224 & 1.7 \\
13L & 50 & 7 & 0.171 & 1.5 \\
16L & 7 & 7 & 0.182 & 1.6 \\
6U & 33 & 11 & 0.165 & 1.6 \\
8H & 6 & 4 & 0.118 & 1.2 \\
H (division I) & 94 & 11 & 0.122 & 1.6 \\
H (division II) & 8 & 5 & 0.216 & 2.1 \\
H (pooled) & 102 & 16 & 0.377 & 2.6 \\
L & 447 & 76 & 0.233 & 3.8 \\
U & 38 & 14 & 0.218 & 2.1 \\
$\quad$ Total & 587 & 103 & 0.319 & 4.4 \\
\hline
\end{tabular}




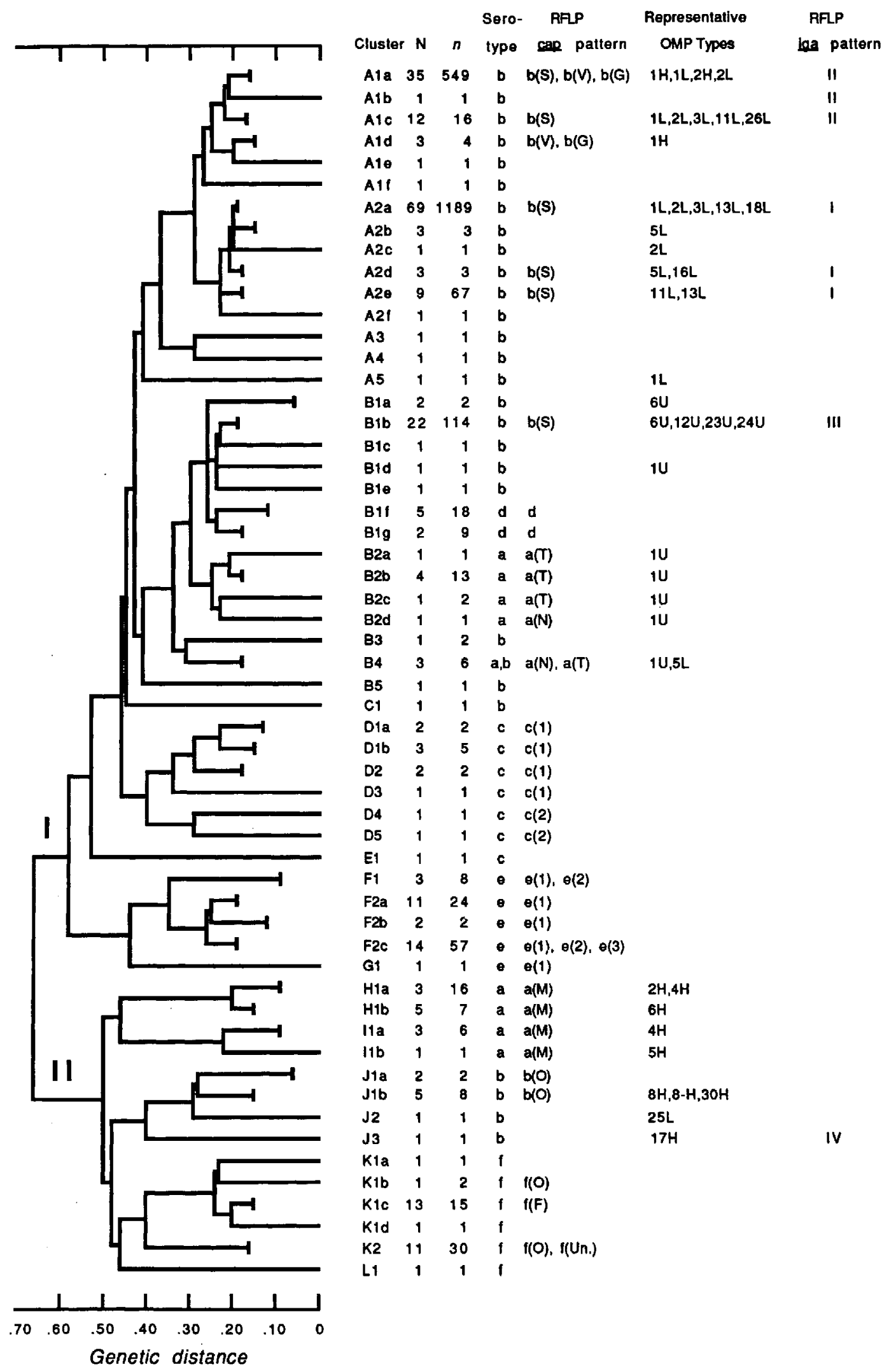




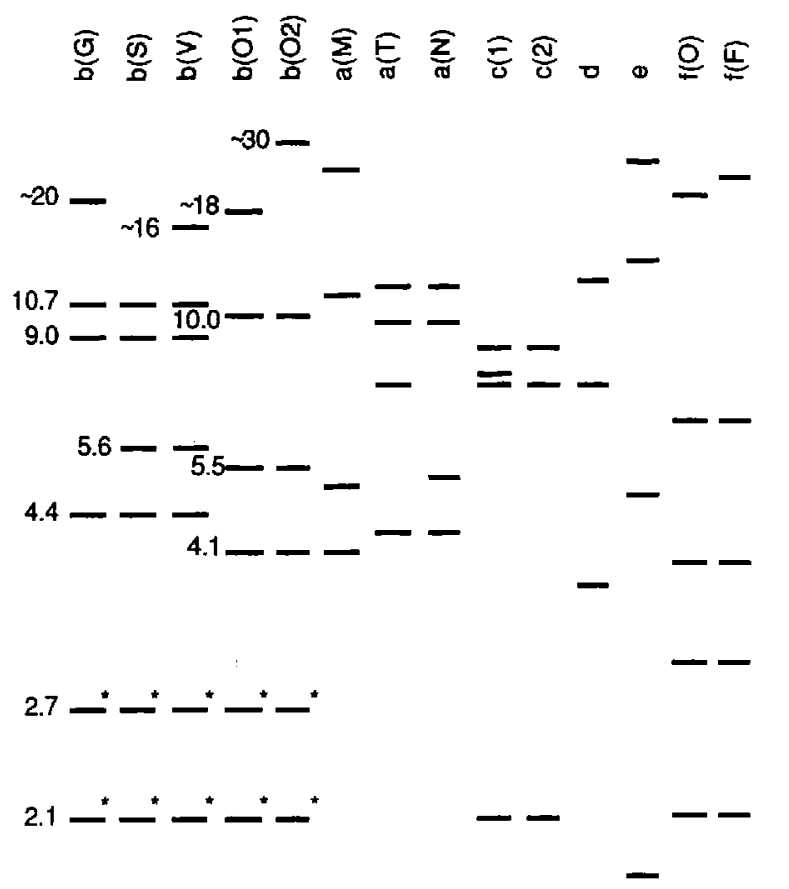

Figure 2. RFLP patterns of the cap region in encapsulated $H$. influenzae. The figure represents a Southern blot of EcoRI-digested chromosomal DNA from the known variants of the six serotypes hybridized to pUO38 [31], a DNA probe containing the entire cap locus from a serotype b strain. Bands marked with an asterisk denote DNA confined to serotype b strains. The sizes of fragments identified in serotype b strains are shown in kilobases. Similarity in size in strains of different serotype does not carry the implication of nucleotide sequence identity.

(table 6). This limited genetic diversity within P1 types (especially OMP subtype $1 \mathrm{H}$ ) reflects the fact that isolates of the same OMP type are likely to be closely similar or identical in overall genetic character, a conclusion earlier deduced from observed associations between OMP type and biotype and between OMP type and ET in a sample from the United States [20].
Table 7. Proportion of ETs shared between pairs of serotype b $H$. influenzae isolates of various OMP patterns.

\begin{tabular}{|c|c|c|c|c|c|c|c|c|c|}
\hline \multirow{2}{*}{$\begin{array}{l}\text { OMP } \\
\text { pat- } \\
\text { tern }\end{array}$} & \multicolumn{9}{|c|}{ OMP pattern } \\
\hline & $1 \mathrm{H}$ & $1 \mathrm{~L}$ & $2 \mathrm{~L}$ & $3 \mathrm{~L}$ & $11 \mathrm{~L}$ & $13 \mathrm{~L}$ & $16 \mathrm{~L}$ & $6 \mathrm{U}$ & $8 \mathrm{H}$ \\
\hline $1 \mathrm{H}$ & - & 0.074 & 0.091 & 0.000 & 0.067 & 0.000 & 0.000 & 0.000 & 0.000 \\
\hline $1 \mathrm{~L}$ & & - & 0.065 & 0.043 & 0.042 & 0.040 & 0.083 & 0.000 & 0.000 \\
\hline $2 \mathrm{~L}$ & & & - & 0.023 & 0.053 & 0.050 & 0.050 & 0.000 & 0.000 \\
\hline $3 \mathrm{~L}$ & & & & - & 0.029 & 0.088 & 0.028 & 0.000 & 0.000 \\
\hline $11 \mathrm{~L}$ & & & & & - & 0.083 & 0.000 & 0.000 & 0.000 \\
\hline $13 \mathrm{~L}$ & & & & & & - & 0.077 & 0.000 & 0.000 \\
\hline $16 \mathrm{~L}$ & & & & & & & - & 0.000 & 0.000 \\
\hline $6 \mathrm{U}$ & & & & & & & & - & 0.000 \\
\hline $8 \mathrm{H}$ & & & & & & & & & - \\
\hline
\end{tabular}

Nine OMP types were represented by isolates assigned to four or more ETs, and the largest number of ETs for these nine classes was recorded for OMP $3 \mathrm{~L}$, which occurred in isolates of $30 \mathrm{ETs}$. However, most OMP subtypes were confined to isolates of ETs in only one or two groups of serotype b strains; exceptions were subtypes $1 \mathrm{~L}, 2 \mathrm{~L}, 3 \mathrm{~L}$, and $11 \mathrm{~L}$, which occurred in isolates assigned to $5,4,3$, and 4 groups, respectively, and there was extensive sharing of ETs among these and other OMP subtypes (table 7).

The common OMP subtypes were $1 \mathrm{H}(13 \%$ of isolates), 1L (10\%), 2L (22\%), 3L (29\%), 13L (8\%), and $6 \mathrm{U}(5 \%)$. Seventy-nine $(99 \%)$ of the $1 \mathrm{H}$ isolates were ET 1.9 or other closely related ETs in group Ala; $98 \%$ of $3 \mathrm{~L}$ isolates were of ETs in group A2a; and $97 \%$ of $6 \mathrm{U}$ isolates were assigned to group Blb. OMP subtypes $1 \mathrm{~L}$ and $2 \mathrm{~L}$ were unusual in that they occurred in significant proportions of isolates assigned to two different clusters (A1 and A2) of the dendrogram: for $1 \mathrm{~L}$ isolates, $50 \%$ and $43 \%$, respectively, were in groups $\mathrm{Ala}$ and $\mathrm{A} 2 \mathrm{a}$; and $2 \mathrm{~L}$ isolates occurred in groups Ala (88\%) and A2a (12\%).

In summary, with a single exception, all isolates assigned to cluster $\mathrm{Al}$ were of OMP types with the $\mathrm{Pl}$ protein designated $\mathrm{H}$ or $\mathrm{L}$; in contrast, all iso-

Figure 1. Dendrogram showing genetic relationships of the 280 ETs of encapsulated $H$. influenzae and serotypes, cap tegion RFLP patterns, common OMP subtypes, and iga RFLP patterns for the various lineages. The dendrogram was generated by the average-linkage method of clustering from a matrix of coefficients of pairwise genetic distance, based on 17 enzyme loci. There are two primary divisions (I and II) and 12 major lineages (A through L). Groups represented by multiple ETs diverging from one another at a genetic distance of 0.20 or less were truncated; the point of truncation indicates the deepest level of divergence of families of ETs within the cluster. The number of ETs (N) and number of isolates $(n)$ in each group are indicated. For serotype a and serotype b isolates, all OMP subtypes occurring in association with each RFLP pattern in each group are indicated, except for clusters A1 and A2, for which several additional OMP subtypes have been identified [20, 21]. The distribution of the iga RFLPs is based upon data in [110]. Note that serotype $a$ and $b$ strains with the same OMP subtype designation may not be identical in actual OMP electrophoretic pattern (see Materials and Methods). Additional OMP subtypes identified in each group are listed in table 4. 
lates in cluster A2 had the L OMP variant, and all isolates in cluster $\mathrm{B} 1$ had the $\mathrm{U}$ variant. The $\mathrm{H}$ band also was present in isolates assigned to clusters $\mathrm{J} 1$ and $\mathbf{J 3}$, and the single strains comprising cluster $\mathrm{J} 2$ had the $L$ variant. We identified 76 ETs among isolates expressing the $\mathrm{L}$ variant, $17 \mathrm{ETs}$ among $\mathrm{H}$ variant strains, and 14 ETs among strains typed as $U$.

\section{Genotypic Diversity Within OMP Patterns of Serotype a Strains}

The distribution of OMP patterns of serotype a isolates was reported previously [21]. Briefly, all isolates of the seven ETs in cluster B2 were OMP type 1U; of the five serotype a isolates in cluster B4, four were OMP subtype $5 \mathrm{~L}$ and the fifth was OMP $1 \mathrm{U}$.

All 30 serotype a isolates in lineages $\mathrm{H}$ and $\mathrm{I}$ in division $\mathrm{II}$ had the $\mathrm{H}$ mobility variant of the $45-\mathrm{kDa}$ heat-modifiable OMP. The most common OMP subtype among the 23 type a isolates in cluster $\mathrm{H} 1$ was $2 \mathrm{H}$, which occurred in 12 isolates; one, seven, and three isolates had OMP subtypes $4 \mathrm{H}, 6 \mathrm{H}$, and $7 \mathrm{H}$, respectively. Three OMP patterns were identified among the seven serotype a isolates in lineage I1: $4 \mathrm{H}$ (five isolates) and $5 \mathrm{H}$ and $8 \mathrm{H}$ (one isolate each).

In sum, all isolates of serotype a assigned to ETs in division $\mathrm{I}$ had the heat-modifiable $\mathrm{U}$ or $\mathrm{L}$ mobility variant of the $45-\mathrm{kDa}$ OMP, and all type a isolates in division $\mathrm{II}$ had the $\mathrm{H}$ mobility variant of this protein.

\section{Genetic Diversity in Relation to Disease Type}

Because a variety of serious diseases are caused by strains of $H$. influenzae [3, 38], we were interested in determining the extent of association of the phylogenetically diverse types with particular diseases and anatomic sites of infection. Our analysis revealed no strong statistical association on a global or continental level between ETs and disease type or anatomic site of recovery of case isolates, but in Finland there was a tendency for serotype $b$ strains of ET 21.8 to cause more meningitis and less epiglottitis compared with stains of the ET 12 clone family $(P=.078$, Fisher's exact test $)[39,40]$. However, this trend was not observed among isolates of ET 21.8 from other parts of the world.

Ten of 22 serotype a isolates of ETs in clusters B2 and B4 (in division I), which are allied with abundant invasive clones of serotype $b$, were recovered from invasive episodes, whereas only one of 30 isolates of ETs in clusters $\mathrm{Hl}$ and I1 (in division II), which are related to lines of serotype $b$ ETs and serotype $\mathrm{f}$ ETs that are rarely recovered from invasive infections, was obtained from a normally sterile body fluid (table 8).

\section{Serotype b Isolates from Native Human Populations} and Patients of Known Ethnic Background

Three samples of isolates recovered from native North American populations were analyzed. A sample of $\mathbf{8 7}$ isolates from Alaska, which was collected as part of an efficacy trial of serotype $b$ conjugate vaccine [41], included strains from Yupik and Inuit Eskimos, Amerinds, and individuals with mixed Native and non-Native American ancestry. Detailed epidemiologic data and strain characterization will be presented elsewhere (J. I. W. and J. M. M., manuscript in preparation). In brief it was found that: (1) among nonnatives in Alaska there were relatively minor differences in ET and OMP subtypes compared with strains recovered from elsewhere in North America, (2) strains of ET 1.9 were more frequently recovered from invasive disease in nonnative (predominantly Caucasian) individuals than in natives, (3) isolates of certain rare ETs were recovered from native populations, often only in isolated geographic areas, and (4) there is considerable variation among the various populations in the clones recovered from asymptomatic carriers and patients with invasive disease. The most striking observation was that 11 of the 12 isolates identified as ET $21.6 / \mathrm{OMP} 13 \mathrm{~L}$ were carrier strains [42].

A sample of 141 isolates from Manitoba included 72 isolates from patients whose ethnic backgrounds were known: 52 strains were from Caucasians, 18 from Amerinds, and one each was from an Inuit and an "oriental" patient. Although $25 \%$ of these isolates were recovered from Amerinds, only $13 \%$ were identified as ET 1.9 (two isolates each from patients

Table 8. Genetic structure in relation to pathogenicity for serotype a isolates of $H$. influenzae.

\begin{tabular}{lcc} 
& \multicolumn{2}{c}{ No. of isolates } \\
\cline { 2 - 3 } & Invasive & Noninvasive \\
\hline Division I & 10 & 12 \\
Division II & 1 & 29 \\
\hline
\end{tabular}

NOTE. $P<.001$ 
with meningitis and cellulitis). Four of the six isolates of ET 25.6 were recovered from Amerindian patients with meningitis; each patient lived in a different part of the province (one isolate each from Garden Hill, Sandy Lake, St. Theresa Point, and Winnipeg).

Thirty-two isolates (21 invasive and 11 carrier strains) recovered from White Mountain Apache Indians living on the Fort Apache Indian Reservation in Arizona [43] were studied. Twelve invasive isolates and six carrier isolates were ET 1.9, and five invasive and five carrier isolates were ET 21.6; of the remaining isolates, all of which were recovered from invasive episodes, two were ET 122, and one each was ET 25.6 and ET 32. The cap region RFLP pattern was determined for 15 of the ET 1.9 isolates: 12 had the $b(V)$ pattern (and, hence most certainly are not OMP pattern $1 \mathrm{H}$, which is charcteristic of most United States ET 1.9 isolates), and three were typed as $b(\mathbf{S})$.

The collection included 12 isolates from Japan: nine isolates were ET 12.8, two isolates were ET 12.9, and one isolate was assigned to ET 172.

Eighty-one isolates from patients in Malaysia were analyzed: $\mathbf{4 0}$ were obtained from Asian Indians, 24 from Malays, 15 from Chinese, and two from individuals whose ethnicity was not recorded. Twentyseven of the isolates $(33 \%)$ ) were obtained from blood or CSF, including 10 isolates from Malays, nine from Asian Indians, and eight from Chinese patients. Most of the remaining isolates were cultured from sputum, usually from patients with lower respiratory tract infection. For these individuals, the serotype b strains were recovered as a pure culture and are presumed to be the etiologic agent. The ethnic background of the patients and ETs of the blood and CSF isolates were as follows: ethnic Malays, six isolates of ET 12.8 and one isolate each of ET 60, ET 99, ET 132, and ET 25.6; Asian Indians, six isolates of ET 12.8 and one isolate each of ET 1.8, ET 60, and ET 25.6; and Chinese, six isolates of ET 12.8 and one isolate each of ET 60 and ET 21.8.

Among the 50 isolates associated with invasive episodes in the Republic of South Africa, 24 were obtained from blacks, two from "colored" individuals, and three from whites; the remainder were from patients of unknown ethnicity. The isolates from blacks included ET 8 (two isolates), ET 12.8 (two isolates), ET 105 (two isolates), ET 141 (four isolates), ET 152 (eight isolates), and one isolate each was ET 60, ET 68, ET 12.5, ET 109, ET 115, ET 130, and ET 153.
The two isolates from colored patients were of ET 12.8 and ET 74, and the isolates from white patients each represented a unique ET, these being ET 35 , ET 76, and ET 12.5.

\section{Genotypic Variation in Relation to Geographic Source}

Of the total of 1,975 serotype $b$ isolates examined, $1,609(81 \%)$ were assigned to one of the following nine ETs, all of which were represented by 20 or more isolates: ETs 1.9, 4, 12.5, 12.7, 12.8, 12.9, 21.6, 21.8, and 25.6 (table 9); the distribution by country of isolates of these nine ETs is shown in table 10.

At least three of these common ETs (ET 1.9, 12.5, and 12.8), and perhaps most of them, have very wide, even global, distributions. However, for each geographic region, our analysis revealed the presence of only one or two common ETs. For example, isolates of ET 1.9 have been recovered in 12 states in the continental United States, eight provinces of Canada, eight countries in western Europe, and in Mexico, the Dominican Republic, Hawaii, Papua New Guinea, the Republic of South Africa, and Australia. Of the 934 North American isolates, 379 (41\%) were ET 1.9 and $151(16 \%)$ were ET 12.8; no other single ET accounted for more than $10 \%$ of the isolates from this continent. Fifty-six percent of the 622 European isolates were ET 12.5 , and $13 \%$ were ET 12.8. The commonest clone among Asian isolates was ET 12.8 $(64 \%)$, and isolates of this ET were also the commonest multilocus genotype recovered in Africa, accounting for $31 \%$ of strains from that continent. Papua New Guinea was most unusual in that ET 25.6 was represented by $43 \%$ of isolates; ET 12.8 also was commonly recovered, accounting for $37 \%$ of isolates.

For serotype b strains, 68 ETs were represented by two or more isolates (mean 3.0). Thirty-four ETs $(50 \%)$ were represented in two or more geographic source groups: 17 ETs from two areas, eight from three areas, five from four areas, and three from five areas; ET 12.8 was represented in all six geographic source groups. However, there is marked geographic variation in genotype representation (table 11). For example, $84 \%(n=379)$ of strains of ET 1.9 were recovered in North America, and 70\% $(n=348)$ of strains of ET 12.5 were from patients in Europe. Similarly, $30(94 \%), 64(84 \%)$, and $51(89 \%)$ of the strains of ET 4, ET 12.7, and ET 21.6, respectively, were collected in North America (table 9).

Iceland was unusual compared with other European countries studied in that $82 \%$ of isolates were 
Table 9. ETs of serotype b $H$. influenzae represented by 20 or more isolates, grouped by geographic source.

\begin{tabular}{|c|c|c|c|c|c|c|c|}
\hline \multirow[b]{2}{*}{ ET } & \multicolumn{6}{|c|}{ No. $(\%)$ of isolates from indicated geographic source } & \multirow[b]{2}{*}{$\begin{array}{c}\text { Total } \\
(n=1,975)\end{array}$} \\
\hline & $\begin{array}{c}\text { North } \\
\text { America } \\
(n=934)\end{array}$ & $\begin{array}{c}\text { Europe* } \\
(n=622)\end{array}$ & $\begin{array}{c}\text { Asia } \\
(n=121)\end{array}$ & $\begin{array}{c}\begin{array}{c}\text { Africa } \\
(n=95)\end{array} \\
\end{array}$ & $\begin{array}{c}\text { Papua } \\
\text { New Guinea } \\
(n=60)\end{array}$ & $\begin{array}{c}\text { Other } \\
(n=143)\end{array}$ & \\
\hline 1.9 & $379(41)$ & $56(9)$ & 0 & $2(2)$ & $1(2)$ & $12(8)$ & $450(23)$ \\
\hline 4 & $30(3)$ & $2(<1)$ & 0 & 0 & 0 & 0 & $32(2)$ \\
\hline 12.5 & $86(9)$ & $348(56)$ & 0 & $3(3)$ & $2(3)$ & $58(41)$ & 497 (25) \\
\hline 12.7 & $64(7)$ & $8(1)$ & $1(<1)$ & 0 & 0 & $3(2)$ & $76(4)$ \\
\hline 12.8 & $151(16)$ & $81(13)$ & $77(64)$ & $29(31)$ & $22(37)$ & $32(22)$ & $392(20)$ \\
\hline 12.9 & $1(<1)$ & $14(2)$ & $7(6)$ & 0 & 0 & $1(<1)$ & $23(1)$ \\
\hline 21.6 & $51(5)$ & $1(<1)$ & $2(2)$ & 0 & $1(2)$ & $2(1)$ & $57(3)$ \\
\hline 21.8 & $4(<1)$ & $24(4)$ & $6(5)$ & 0 & 0 & $1(<1)$ & $35(2)$ \\
\hline 25.6 & $16(2)$ & 0 & $5(4)$ & 0 & $26(43)$ & 0 & $47(2)$ \\
\hline Other & $152(16)$ & $88(14)$ & 23 (19) & $61(64)$ & $8(13)$ & $34(24)$ & $366(18)$ \\
\hline
\end{tabular}

* If isolates from Iceland are excluded, the data for Europe are: ET $1.9(4 \%), 4(1 \%), 12.5(60 \%), 12.7(1 \%), 12.8(13 \%), 12.9(2 \%)$, $21.6(1 \%), 21.8(4 \%), 25.6(0)$, and other $(15 \%)$.

Table 10. Distribution by country of $H$. influenzae serotype b ETs represented by 20 or more isolates.

\begin{tabular}{|c|c|c|c|c|c|c|c|c|c|c|c|}
\hline \multirow[b]{2}{*}{ Geographic source } & \multirow[b]{2}{*}{$n$} & \multicolumn{10}{|c|}{ Percentage of total sample represented by each ET } \\
\hline & & $\begin{array}{c}1.9 \\
(23)\end{array}$ & $\begin{array}{c}4 \\
(2) \\
\end{array}$ & $\begin{array}{l}12.5 \\
(25) \\
\end{array}$ & $\begin{array}{c}12.7 \\
(4) \\
\end{array}$ & $\begin{array}{l}12.8 \\
(20) \\
\end{array}$ & $\begin{array}{c}12.9 \\
\text { (1) }\end{array}$ & $\begin{array}{c}21.6 \\
(3) \\
\end{array}$ & $\begin{array}{c}21.8 \\
(2)\end{array}$ & $\begin{array}{l}25.6 \\
(2)\end{array}$ & $\begin{array}{l}\text { Other } \\
\text { (18) }\end{array}$ \\
\hline Australia & 40 & 7 & & 60 & 2 & 5 & & & & & 26 \\
\hline Canada & 375 & 38 & 1 & 17 & 6 & 19 & $<1$ & $<1$ & & 2 & 24 \\
\hline Denmark & 43 & 7 & & 53 & 2 & 4 & & 2 & & & 34 \\
\hline Dominican Republic & 55 & 2 & & 33 & & 36 & $<1$ & & 2 & & 26 \\
\hline England & 21 & 14 & & 57 & 5 & 5 & & & & & 19 \\
\hline Finland & 100 & 2 & & 23 & & 44 & 3 & & 19 & & 9 \\
\hline France & 77 & 1 & & 78 & & 6 & 1 & & & & 14 \\
\hline The Gambia & 30 & & & & & 60 & & & & & 40 \\
\hline Iceland & 40 & 82 & 2 & 7 & & & & & 2 & & 7 \\
\hline Malaysia & 81 & & & & 1 & 64 & & & 7 & 5 & 23 \\
\hline Norway & 37 & 16 & & 43 & 5 & 14 & 1 & & & & 21 \\
\hline Papua New Guinea & 60 & 2 & & 3 & & 37 & & & 2 & 43 & 13 \\
\hline Republic of South Africa & 50 & 4 & & 6 & & 18 & & & & & 72 \\
\hline Scotland & 20 & & & 65 & & 5 & & & & & 30 \\
\hline Spain & 71 & & & 97 & & & & & & & 3 \\
\hline Sweden & 78 & 8 & 1 & 44 & & 18 & 6 & & 5 & & 18 \\
\hline Switzerland & 123 & 2 & & 72 & 3 & 7 & 2 & & & & 14 \\
\hline United States & 472 & 44 & 4 & 4 & 6 & 16 & & 8 & $<1$ & 2 & 15 \\
\hline
\end{tabular}

Table 11. Proportion of ETs shared between pairs of serotype $\mathrm{b} H$. influenzae isolates from various geographic source groups.

\begin{tabular}{|c|c|c|c|c|c|c|}
\hline \multirow[b]{2}{*}{ Source group } & \multicolumn{6}{|c|}{ Source group } \\
\hline & $\begin{array}{l}\text { North } \\
\text { America }\end{array}$ & Europe & Asia & \multicolumn{3}{|c|}{$\begin{array}{l}\text { Papua } \\
\text { New }\end{array}$} \\
\hline North America & - & 0.155 & 0.061 & 0.048 & 0.056 & 0.118 \\
\hline Europe & & - & 0.116 & 0.130 & 0.078 & 0.156 \\
\hline Asia & & & - & 0.073 & 0.130 & 0.150 \\
\hline Africa & & & & - & 0.091 & 0.098 \\
\hline Papua New Guir & inea & & & & - & 0.152 \\
\hline Other & & & & & & - \\
\hline
\end{tabular}

ET 1.9; and, of these strains, 94\% were OMP 2L. One strain of OMP $1 \mathrm{H}$ was identified. The cap region RFLP pattern was determined for three isolates classified as ET 1.9/OMP $2 \mathrm{~L}$ and the single strain of ET 1.9/OMP 1H: two of the ET 1.9/2L isolates had the $b(S)$ pattern, and the third isolate was typed as $\mathrm{b}(\mathrm{V})$; the ET $1.9 / 1 \mathrm{H}$ isolates had the $\mathrm{b}(\mathrm{G})$ cap region pattern. The ET $1.9 / 1 \mathrm{H} / \mathrm{b}(\mathrm{G})$ strain was recovered from a 5-year-old American child with epiglottitis who lived on the U.S. naval base at Keflavik. The remaining isolates of ET 1.9 were cultured from patients with invasive disease living in communities throughout Iceland: Reykjavik (18 isolates), Akureyri 
(three isolates), Kopavogur (two isolates), Keflavik town (two isolates), and one isolate each was from Husavik, Eskifjordur, Djupivogur, Egilsstadir, Budardalur, Vestmannaeyjar, and Gardabaer. Five additional strains were recovered from individuals in Reykjavik (two were ET 12.5, and one each was assigned to ET 4, ET 72, and ET 175), one strain was from Breiddalsvik (ET 12.5), and one isolate of ET 21.8 was from a patient in Hafnarfjorour.

Variation in the frequency of certain ETs also occurred between geographically contiguous countries that are closely allied historically, ethnically, and politically. One striking example, for serotype $b$ isolates, was the occurrence of ET 100 (17 isolates) throughout Canada (Ontario, Manitoba, Alberta, and British Columbia) but its absence in the sample from the United States. Similarly, $17 \%$ of Canadian isolates, but only $4 \%$ of isolates from the United States, were ET 12.5.

Geographic variation in frequency was also recorded for ETs of isolates of other capsule serotypes; in many cases this was a result of the occurrence of one or a few "private" alleles [44] only in certain areas. For example, the two serotype e isolates from Kenya (ET 205) in the collection were distinguished from isolates assigned to the common serotype $e$ genotype (ET 204) only by the occurrrence of a different allele at the PGM locus. Similarly, all serotype $d$ isolates from Malaysia were represented by ET 162, which differs at two loci (PE1 and CAT) from the common clone (ET 157) of serotype $d$ from the United Kingdom (table 2).

In summary, there is striking geographic variation in the frequency of recovery of certain multilocus genotypes. For serotype b organisms, ET 12.8 was frequently recovered in all regions, whereas isolates of ET 1.9, ET 12.5, and ET 25.6 were common only in single geographic source groups.

\section{Serotype b Isolates from Asymptomatic Carriers}

Isolates of seven distinctive multilocus genotypes were recovered from asymptomatic carriers. Of these isolates, seven were ET 1.9 (including five from Amerinds in Arizona), seven were ET 12.8 (United States, South Korea, and Canada), five were ET 12.9 (South Korea), two were ET 12.7 (Alaskan Natives), 47 were ET 12.5 (including 46 from associated individuals in three day care centers in Spain; [28]), five were ET 103 (Alaskan Natives), and 16 were ET 21.6
(11 from Alaskan Natives and five from White Mountain Apaches in Arizona).

\section{Discussion}

For purposes of reference and discussion, ETs are considered to mark clones, and isolates of a given ET with different OMP types, cap region RFLP patterns, biotypes, or LPS serotypes are regarded as subclones $[20,21]$.

\section{Estimating Genetic Diversity and Relatedness Among Isolates}

For Escherichia coli and Shigella species [45], Legionella species [46, 47], Bordetella species [48], Salmonella species (R. K. S., unpublished data), Gluconobacter species [49], the oral streptococci [50], Listeria species (J.-C. P., unpublished data), other bacteria [51, 52], and many higher organisms [53-55], estimates of genetic relatedness based on multilocus enzyme electrophoresis have been shown to be strongly correlated with measures of similarity in chromosomal nucleotide sequence derived from DNA hybridization experiments. Therefore, there is reason to believe that the 17 enzyme loci examined in this study are a representative sample of encapsulated $\boldsymbol{H}$. influenzae genomes and, hence, provide a basis of estimating both overall genetic relationships among strains and levels of genetic diversity within populations.

\section{Nature of the Sample Studied}

Most of the strains analyzed were recovered in countries with modern bacteriology laboratories and strong affiliation with the United States or European countries. Samples from these countries were recovered from a very large number of different geographic sources (table 1) (and in some cases from two or more hospitals in the same community), and no consistent bias in isolate selection was identified. Hence, we are confident that the serotype b samples are generally representative of natural populations of medically important $H$. influenzae. This is especially true for the samples from Alaska and Finland, both of which were collected as part of populationbased epidemiologic studies performed in conjunction with vaccine efficacy trials $[41,56]$ (a random sample of strains recovered from each area was analyzed), and from other regions represented by rela- 
tively large samples (e.g., Canada, Switzerland, and Malaysia). Numerous attempts were made, without success, to obtain isolates from parts of the world not represented in the collection but of particular interest, such as the USSR, the People's Republic of China, India, and Japan (see beyond). Because the clonal composition of populations of $H$. influenzae varies geographically, additional ETs of encapsulated isolates undoubtedly exist in parts of the world that have yet to be sampled, but further sampling is not expected to affect the qualitative aspects of our findings.

\section{Variation in Serotype in Relation to Population Structure}

Although capsular polysaccharide serotypes clearly do not mark individual clones of $H$. influenzae, two lines of evidence demonstrate that capsule type has some relation to the phylogenetic structure of the species [21, 22]. First, none of the 280 ETs identified was represented by isolates of more than one serotype. Second, isolates expressing each of the six capsule polysaccharides belong to only one or a few major phylogenetic lineages, which in certain cases are strongly differentiated from the lineages of all other capsule types. This restriction accounts for the fact that mean genetic diversity per enzyme locus among ETs within serotypes was, on average, only two-thirds that recorded for all ETs. The latter observation, in conjunction with other data [24], is most parsimoniously interpreted as evidence that the common clones of each serotype were derived from a relatively small number of ancestral clones. In the case of serotype $b$ isolates, this number is probably three or four, depending upon the number of horizontal gene transfer events that gave rise to serotype $b$ isolates in phylogenetic line II (see beyond) [22].

Modal multilocus genotypes (ETs) of strains of the major lineages of each serotype are shown in table 12. These were constucted by taking the most frequent allele among ETs at each of the 17 enzyme loci assayed. (Because the modal multilocus genotypes were inferred on the basis of allele frequencies among ETs rather than isolates, they are not influenced by variation in the frequency of isolation of the ETs.) For serotype $b$, the modal ETs for the three major lineages of division I are the commonly recorded genotypes designated as ETs 1.9, 12.8, and 25.6, which may be interpreted as evidence that these genotypes are genetically similar to the three ancestral clones from which other clones of type $b$ isolates have diverged.

In the laboratory, a type b strain can be transformed to express each of the other five capsule types with chromosomal DNA derived from isolates of the appropriate serotype [57]. Recently it has been shown by restriction enzyme mapping studies that the cap region of representative type $a, b, c$, and d strains is organized in a common fashion [58]. The cap region contains a central DNA segment coding for serotype-specific functions, and DNA probes derived from this region hybridize only to chromosomal DNA from isolates of the same capsule type: they are serotype specific [58]. The sizes of these regions are estimated to be (in kilobases) 3.2-5.4 (serotype a), 5.8-8.2 (serotype b), 5.3-7.7 (serotype c), and

Table 12. Modal ETs of common strains of encapsulated $H$. influenzae.

\begin{tabular}{|c|c|c|c|c|c|c|c|c|c|c|c|c|c|c|c|c|c|}
\hline \multirow[b]{2}{*}{ Serotype } & \multicolumn{17}{|c|}{ Allele at indicated enzyme locus } \\
\hline & CAK & NSP & PGI & MAE & MDH & G6P & GOT & ADK & 6PG & PE2 & PE1 & LAP & PGM & CAT & GLD & G3P & FUM \\
\hline a (division I) & 6 & 5 & 5 & 3 & 5 & 6 & 3 & 2 & 2 & 4 & 5 & 2 & 1 & 2 & 4 & 2 & 3 \\
\hline a (division II) & 7 & 5 & 4 & 1 & 5 & 1 & 3 & 2 & 5 & 2 & 3 & 2 & 4 & 3 & 1 & 2 & 2 \\
\hline b (lineage $\mathrm{Al}$ ) & 9 & 5 & 1 & 5 & 5 & 3 & 5 & 2 & 2 & 4 & 5 & 2 & 4 & 2 & 4 & 2 & 2 \\
\hline b (lineages A2-A5) & 8 & 5 & 4 & 5 & 5 & 3 & 3 & 2 & 2 & 4 & 5 & 6 & 4 & 2 & 4 & 2 & 2 \\
\hline b (lineages B1-C1) & 6 & 5 & 5 & 3 & 3 & 6 & 3 & 2 & 2 & 4 & 5 & 3 & 6 & 2 & 4 & 2 & 2 \\
\hline b (lineages J1b-J3) & 7 & 5 & 4 & 2 & 5 & 1 & 3 & 1 & 4 & 3 & 5 & 2 & 1 & 3 & 4 & 2 & 2 \\
\hline c & 6 & 5 & 10 & 5 & 1 & 6 & 5 & 2 & 2 & 4 & 1 & 2 & 6 & 2 & 4 & 2 & 2 \\
\hline d & 6 & 5 & 5 & 3 & 5 & 6 & 3 & 2 & 2 & 4 & 5 & 3 & 6 & 2 & 4 & 2 & 2 \\
\hline e & 9 & 5 & 9 & 2 & 5 & 3 & 3 & 2 & 2 & 2 & 3 & 3 & 7 & 2 & 5 & 2 & 2 \\
\hline f & 7 & 5 & 2 & 2 & 5 & 1 & 3 & 1 & 5 & 2 & 2 & 3 & 4 & 3 & 4 & 2 & 2 \\
\hline
\end{tabular}

NOTE. Modal ETs are combinations of the most common allele at each of the 17 enzyme loci. Enzyme abbreviations are defined in footnote to table 3 . 
5.3-8.7 (serotype d). The cap regions in serotype e and serotype $f$ strains have not yet been examined in as much detail.

In areas flanking the serotype-specific region, there is great conservation of restriction endonuclease cleavage maps. Hence, the physical arrangement of chromosomal loci for capsulation functions in strains of these four serotypes is very uniform, and it has been suggested that the cap region is organized in such a way that exchange of central region DNA by horizontal gene transfer and recombination will lead to alteration of capsule structure [58]. It is highly probable that horizontal gene transfer of the central region of cap has been important in generating a small number of numerically unsuccessful cell lines of minimal clinical significance in phylogenetic line II [22]. However, because our analysis revealed that individual ETs are comprised solely of isolates expressing only a single capsule polysaccharide, we infer that alteration of capsule structure as a result of horizontal transfer of the serotypespecific region of the cap locus rarely occurs in natural populations. The observation that many type $b$ isolates from invasive episodes fail to undergo DNA-mediated transformation in the laboratory [59] is consistent with this notion.

\section{Variation in OMP in Relation to Population Structure}

The OMP electrophoretic typing system was developed to classify serotype $b$ and $a$ isolates for epidemiologic studies [8, 18, 19]. For isolates of these serotypes, there are strong nonrandom associations between OMP type and ET, with little sharing of OMP subtypes between isolates of ETs in different phylogenetic clusters [20, 21]. However, as demonstrated here, individual OMP patterns may occur in genetically diverse clones. For the nine common serotype b OMP subtypes represented in the sample, mean genetic diversity was, on average, $53 \%$ of that in the total sample of serotype b isolates, with an average of nine ETs per OMP subtype. The practical significance of this finding for epidemiologic studies is that the grouping of strains solely on the basis of OMP subtype may lead to erroneous conclusions concerning strain relationships. This is especially true for subtypes $1 \mathrm{~L}$ and $2 \mathrm{~L}$, which commonly occur in two different clone families. The occurrence of OMP subtypes $1 \mathrm{~L}$ and $2 \mathrm{~L}$ in genetically divergent clones means that these patterns do not consistently mark distinct multilocus genotypes.
In contrast, there is strong statistical association of OMP subtypes $1 \mathrm{H}$ and $6 \mathrm{U}$ with certain multilocus enzyme genotypes, and isolates expressing OMP type $3 \mathrm{~L}$, although confined predominantly to lineage A2a (figure 1), are represented by 30 distinct ETs (table 6). Hence, until better methods are available, finestructure epidemiologic studies should employ a combination of ET and OMP, ET and cap region RFLP, or ET, OMP, and cap region RFLP analyses.

Considering the abundant genetic diversity in enzyme genes identified among strains of certain OMP patterns, it is likely that the structural genes coding for individual OMPs of the same electrophoretic mobility are also polymorphic. Consistent with this notion is the recent observation [60] that OMPs of type b isolates with identical apparent molecular weights may possess different surface-exposed epitopes. It would clearly be of interest to perform comparative DNA sequencing on genes for individual OMPs selected with reference to the population genetic framework presented here. Studies of this type could answer the question of whether the occurrence of the same OMP pattern in strains belonging to different phylogenetic clusters of the dendrogram represents horizonal transfer events or the retention of an ancestral OMP pattern.

Takala et al. [61] recently reported that in Finland type $b$ strains of OMP subtypes 1 and $1 c$ (3L and IL, respectively, in the system described by Barenkamp [8]) are nonrandomly associated with different types of invasive disease. The observation that strains of subtype lc caused proportionally more meningitis and less epiglottitis than did those of subtype 1 was interpreted as evidence of a true difference in virulence between isolates expressing these OMP types, and it was hypothesized that the subtype lc protein marks a clone with special virulence properties. The present study and others $[39,40]$ have shown that the OMP subtype 1 in Finland is commonly associated with ET 12.5, ET 12.8, and a small number of closely allied clones, whereas subtype lc usually occurs in isolates of ET 21.8 (83\% of isolates [37]), which differs from ET 12.5 and ET 12.8 at two and one loci, respectively, of the 17 enzyme loci assayed. Because all properties tend to be associated in clonal organisms $[34,62]$, the results are consistent with the interpretation that in Finland the type lc outer membrane protein subtype frequently marks a subclone that is especially successful in invading the CSF. However, the occurrence in high frequency of this OMP subtype in strains recovered 
from cases of meningitis does not mean that it, per se, has any role in virulence or organotropism.

\section{Clonal Structure and Antibiotic Resistance}

The strongly clonal population structure identified for encapsulated $H$. influenzae strains, coupled with an apparently relatively slow rate of clone dissemination over wide geographic areas, suggests that regional variation may also occur in antibiotic resistance elements. Although we made no systematic effort to identify antibiotic-resistant strains, the molecular epidemiology of some of the resistant serotype $b$ isolates we examined has been studied by other investigators. Mendelman et al. [63] characterized 36 ampicillin-resistant type $b$ strains recovered from native and nonnative individuals in four geographic regions of Alaska by OMP subtyping and restriction endonuclease plasmid profiling. Of the seven strains harboring detectable extrachromosomal DNA, four had a plasmid with a molecular mass of 40 megadaltons (MDa), and three contained a plasmid of $3 \mathrm{MDa}$. Isolates bearing the 3-MDa plasmid were identified only in the south-central region of the state; the 40-MDa plasmid from isolates originating in central Alaska was distinguishable from all other 40-MDa plasmids in Alaskan strains by restriction endonuclease analysis [63]. Similarly, the 40-MDa plasmids carried by isolates from individuals in northwest Alaska were identical to each other but differed in restriction endonuclease digestion pattern from 40-MDa resistance plasmids from other Alaskan regions. There were three distinct OMP subtypes [63] and nine ETs (data not shown) represented among the ampicillin-resistant strains.

Among the 66 ampicillin-resistant $H$. influenzae organisms from patients in the continental United States studied by Willard et al. [64], four plasmids of various sizes ( $3 \mathrm{MDa}, 30 \mathrm{MDa}, 36 \mathrm{MDa}$, and 50 $\mathrm{MDa}$ ) were identified, but the Alaskan 40-mDa plasmid was not represented.

In a study of the molecular epidemiology of multiply resistant $\boldsymbol{H}$. influenzae type b isolates from carriers and patients attending four day care centers in Spain, restriction endonuclease analysis revealed the presence of at least four distinct plasmids of molecular mass $45 \mathrm{MDa}$ and $52 \mathrm{MDa}$ and three different OMP subtypes [65].

The results from these studies, other reports [66], and unpublished data of the authors of this review indicate that extensive genetic diversity exists among the plasmids encoding antibiotic resistance genes and among the chromosomal genotypes of the resistant type b strains. Hence, unlike one documented case for $E$. coli [67], no single antibiotic-resistant clone has widespread geographic range: antibiotic-resistant determinants have been acquired by type $b$ strains through multiple independent evolutionary events.

\section{Rarity of Significant Associations of Clones and Clinical Syndromes}

One situation was identified in which there was a statistically significant association of a clone with a particular clinical syndrome-ET 21.8 and meningitis in Finland - but no other obvious associations of clones and invasive disease types were revealed. For example, there was no unusual tendency for ET 21.8 to cause meningitis in countries other than Finland; and in Malaysia ET 21.8 may be more frequently associated with respiratory tract disease than meningitis (five of six isolates). However, only for the samples from Finland and Alaska were there available detailed clinical histories for all isolates.

The genetic distances separating the three clinically important major lineages of type b organisms are approximately equivalent to those dividing pig-, dog-, and fowl-specialist clones of Bordetella bronchiseptica [68] and separating clones of Salmonella species exhibiting host specialization [69]. It was therefore somewhat surprising that no strong diseaseclone associations for the major serotype $b$ phylogenetic lineages in division I were found. Although clones of all major serotype $b$ lineages apparently are more or less equivalent in their ability to cause septicemia, meningitis, cellulitis, epiglottitis, or other invasive disease, it may well be that the failure to demonstrate clonal disease specificity was caused by a lack of detailed clinical information for the great majority of strains examined. In many areas sampled, a tendency was observed for association of clones and disease types, but these never reached statistical significance. Moreover, a clone or subclone tending to cause a proportionally greater amount of, for example, meningitis in one geographic area might cause less meningitis and more septicemia, epiglottitis, or other type of disease in another area with a different ethnic patient population. For example, in Pakistan and Papua New Guinea, an unusually high frequency of pneumonia with septicemia is 
caused by strains of the ET 25.6 clone family (ET 141) [70-72], but, among Amerinds in Manitoba, this subclone has a tendency to cause meningitis.

\section{Serotype b Asymptomatic Carrier Isolates}

The demonstration that a number of different multilocus genotypes are associated with asymptomatic serotype b carriage is consistent with data on OMP patterns presented earlier [18]. In general, most ETs represented by carrier isolates were also commonly associated with invasive episodes, but isolates of ET 21.6/OMP $13 \mathrm{~L}$ were rarely recovered from normally sterile body fluids. Of the several hypotheses that could explain these results, we favor the interpretation, advanced previously on the basis of OMP profiling data [18], that ET 21.6/OMP 13L isolates represent a subclone that is "less invasive." Clearly, additional epidemiologic and animal model studies will be required to test this hypothesis.

\section{Clone and Subclone Distribution in Iceland}

The finding that the frequency of common diseasecausing type b clones in Iceland was highly unusual compared with other European countries is consistent with the results of an earlier analysis by van Alphen et al. [23] of OMP profiles, biotypes, and LPS serotypes in a smaller collection of strains. These investigators did not develop a cogent hypothesis to explain the difference in strain subtype distributions. One interpretation is that isolates of ET 1.9/OMP $2 \mathrm{~L}$ represent an "old" clone in Iceland, perhaps introduced by Celts in the course of early settlement of the island and that ET 12.5/OMP 3L has been more recently introduced as a result of population migration and contact with other western European peoples. This hypothesis is based on two lines of evidence.

First, subclone ET 1.9/OMP 2L, which accounted for $77 \%$ of all Icelandic isolates, also was represented by $60 \%$ of isolates from patients in the Canadian Maritime Provinces, where the human population is predominantly of Celtic origin and, like that in Iceland, has experienced little immigration in recent centuries. (Strains from the Maritime Provinces were sought specifically to test this Celtic hypothesis.) Although it is not generally appreciated, genetic evidence based on the frequencies of A-B-O blood group antigens clearly demonstrates that Icelanders are closely allied to Scots and Irish and differ markedly from modern Norwegians and other Scandinavians [73]. The available data indicate that the early Icelandic population (A.D. 950) was a CelticNorse mixture, the exact proportions of which are unknown [74]. Germane to our hypothesis is the observation that the same globally uncommon subclone (ET 1.9/OMP 2L) occurs in high frequency among two human populations that share Celtic ancestry but that diverged relatively long ago and have experienced little immigration even to the present time.

Second, strains of ET 1.9/2L were recovered from patients residing in all parts of Iceland (Reykjavik and nine other communities), whereas ET 12.5/3L was confined to the Reykjavik area (two isolates) and Breiddalsvik (one isolate). Because of travel patterns in Iceland, and by analogy with the spread of measles virus on the island [75], it is most likely that the introduction of new clones of type $b \boldsymbol{H}$. influenzae would occur first in Reykjavik and that subsequently they would be disseminated to smaller villages around the perimeter of the island.

We reject the alternative interpretation, that ET $12.5 / 3 \mathrm{~L}$ is an "old" Icelandic clone and that ET $1.9 / 2 \mathrm{~L}$ is "new," because of the rarity of ET 1.9 isolates in general, and ET 1.9/2L isolates in particular, in other European nations sampled, especially those whose populations would be the likely donors of strains with this ET/OMP combination. Intensive longitudinal sampling of invasive isolates from Icelanders, especially individuals living outside of the Reykjavik area, would help to resolve this issue.

\section{Invasive Disease in Japan}

Early in the study, the repeated recovery of isolates of ET 25.6 and closely allied clones from invasive episodes in Papua New Guinea and the virtual absence of these clones in collections from Europe and North America suggested that ET 25.6 occurs in appreciable frequency only in eastern Asia or the Pacific Basin. We attempted to test this hypothesis in two ways.

First, many requests for type $b$ or other invasive isolates were sent to medical scientists in various countries in this region. An especially intensive effort was made to contact medical microbiologists in India, the People's Republic of China, the Republic of Singapore, and Japan. Unfortunately, however, 
we were unsuccessful in obtaining isolates from India, the People's Republic of China, the Republic of Singapore, and a number of other countries. However, three investigators in Japan provided 12 type $b$ isolates, including four recovered from normally sterile body fluids. Information obtained through correspondence with Drs. T. Konda, A. Wake, Y. Terawaki, Y. Sakata, and S. Uehara suggested that the frequency of invasive $H$. influenzae episodes in most areas in Japan is low and that the rarity of disease does not reflect merely an inability of Japanese medical microbiologists to culture $H$. influenzae $[76,77]$.

However, in Hong Kong, a city whose population is $\mathbf{9 8 \%}$ ethnic Chinese, invasive serotype b disease apparently occurs with a frequency similar to that in Western countries (G. C. French, professor of Microbiology, Chinese University of Hong Kong, personal communication), and this observation is consistent with a report from Malaysia describing somewhat similar frequencies of invasive type $b$ disease in ethnic Malays, Asian Indians, and Chinese living in Kuala Lumpur [78].

Because of the failure to obtain adequate samples of invasive isolates from Japan and other areas listed above, testing of the hypothesis was attempted in another way. The senior author contacted Dr. Arnold L. Smith, Children's Hospital of Seattle, Washington, a city with a very large component of people of oriental ancestry, and requested 40 invasive serotype b strains recovered from individuals with Japanese, Chinese, or other ethnically Asian surnames. Although Dr. Smith has for about 10 years received cultures of almost all isolates of $H$. influenzae recovered from sterile body fluids of patients in King County, Washington (population approximately 1.3 million) and maintains a collection of hundreds of invasive strains from the area, he was able to identify only 11 isolates that had originated from patients with Asian ancestry [79].

Although we fully recognize the dangers of formulating hypotheses on the basis of anecdotal information, we tentatively suggest that the Japanese are less prone to develop invasive $H$. influenzae disease than are Caucasians or certain other ethnic groups. Variation within and among human populations in immunologic response to serotype b capsule polysaccharide and susceptibility to other encapsulated bacterial pathogens has been reported $[80,81]$. It also has been noted that individuals with the nonsecretor phenotype may be at increased risk of developing invasive disease caused by encapsu- lated bacterial pathogens, including $H$. influenzae $[82,83]$. This hypothesis is advanced primarily to stimulate further study of the epidemiologic and immunologic aspects of invasive type $b$ disease in regions of the world and ethnic populations for which currently there is little knowledge.

\section{Evolution of Serotype b Clones}

For reasons noted in Results, we suggest that ET 1.9, ET 12.8, and ET 25.6 are genetically similar to the three primordial clones of serotype $\mathrm{b} H$. influenzae from which other extant clones producing this capsule type originated. According to this hypothesis, these three clones, through accumulation of point mutations, deletions and insertions, and, perhaps, occasional horizontal gene transfer and recombination of chromosomal genes, have generated most of the serotype b ETs represented in the collection. As discussed elsewhere [22], the occurrence of serotype b clones in primary division II represents a rare case of the transfer of b-specific segments of the cap region into evolutionarily old phylogenetic lines.

The combined evidence from estimates of genetic distance (shown in figure 1) and the pattern of sharing of restriction sites in the cap locus indicates that the divergence leading to type $b$ clones in group Blb through cluster B5 from those type b clones in clusters A1 through A5 was an ancient event, occurring perhaps in conjunction with the divergence of Eurasians and Africans $[84,85]$. Three lines of evidence suggest that the ET 12.8 clone is evolutionarily old. First, ET 12.8 is geographically the most widespread of any clone represented in the collection, being the only one recovered in most of the countries sampled in all six geographic source groups; and it has been identified in eight additional countries (J. M. Musser, unpublished data). Second, all ET 12.8 isolates except one have the $b(S)$ cap region RFLP pattern, which is also characteristic of all tested strains of ET 25.6 and closely allied clones and which, based on the pattern of sharing of restriction enzyme recognition sites, probably represents the primordial cap b locus RFLP [22]. Third, there is an abundance of different OMP patterns among isolates of ET 12.8 (table 4), which indicates that this genotype has existed for a sufficient period to generate much sequence diversity in genes coding for OMPs. Taken together, the data are interpreted to mean that ET 12.8 has had a relatively long association with humans and has been dispersed world- 
wide in the course of migrations of aboriginal populations and also during recorded history.

Strains of ET 25.6 and allied type b clones assigned to clusters $\mathrm{Bl}$ through $\mathrm{Cl}$ also apparently are evolutionarily old, as judged by the estimates of genetic distance shown in figure 1 and the presence of the $\mathrm{b}(\mathrm{S})$ cap region RFLP pattern. However, clones of this major type $b$ phylogenetic line are less geographically widespread, are numerically less prominent, and are characterized by possession solely of the $U$ electrophoretic mobility variant of the P1 heatmodifiable OMP.

Two explanations could account for the relative lack of diversity in enzyme-encoded genes and OMPs in conjunction with possession of the "old" cap b region RFLP. First, restricted genetic diversity could be caused by small, effective population size [34], ecologic niche specialization, or a low mutation rate. There is no reason to suspect that clones in this line are any less mutable than other serotype $\mathrm{b} H$. influenzae. Evidence for some degree of niche specialization is provided by the recent discovery that strains of the ET 25.6 clone family were responsible for more than $95 \%$ of episodes of type b pneumonia with sepsis in Pakistan [70, 71], an observation suggesting possible viscerotropism for lung parenchyma. (Statistical association of ET 25.6 clones with pneumonia and sepsis was not recorded in other geographic areas [data not shown]; in Pakistan we have yet to examine a large sample of isolates from children with meningitis.) Small population size is suggested by the relative paucity of isolates of ET 25.6 in the collection and their restricted geographic distribution compared with those of ET 12.8 and many other clones.

A second possibility is that the length of time of association of the cap b locus with this phylogenetic line of $H$. influenzae actually has been much shorter than that implied by the depth of genetic distance shown in figure 1. According to this hypothesis, one or more progenitors (most probably unencapsulated) of phylogenetic lines B1 through $\mathrm{C1}$ relatively recently received some part or all of the cap b region by horizontal transfer and recombination, and the resulting type b strains have not yet had an opportunity to spread globally. Clearly, with the data currently available, elimination of either of these alternative hypotheses is not possible. However, the interpretation that small population size accounts, in large part, for the restricted genetic diversity ob- served among isolates of ET 25.6 and associated lines is favored.

It was hypothesized above that ET 1.9 represents a third primordial clone of serotype $b$ and is an "old" European clone that in relatively recent years has been supplanted by the ET 12.5/OMP 3L subclone. Two observations suggest that ET 12.5/OMP $3 \mathrm{~L}$ has recently spread throughout Europe, most probably in an episode of periodic selection of a mutation affecting fitness [86-88]. First, almost all ET 12.5 isolates have the same biotype (BT I), and most are of a single LPS serotype (serotype 1) [23], which suggests that this subclone has not yet had time to accumulate significant recognizable diversity. Second, as noted, all isolates of ET 12.5 tested were OMP 3L and had the $b(S)$ cap region RFLP pattern (table 4).

The probable displacement of ET 1.9 (most likely ET 1.9/OMP 2L) by subclone ET 12.5/OMP 3L represents an example of clonal replacement in populations of human pathogenic bacteria, first described by Caugant et al. [89] for the ET 5 complex of Neisseria meningitidis, also in Europe. Because of the relatively large genetic distance between ET 1.9 and ET 12.5 (these clones differ at four of 17 metabolic enzyme-encoding loci) and the differences in OMPs between $2 \mathrm{~L}$ and $3 \mathrm{~L}$, the replacement episode is envisioned to be analogous to the phenomenon of antigenic shift identified in influenza virus epidemiology $[90,91]$; by similar analogy, episodes involving temporal variation in OMP subclones represent antigenic drift. Many of the hypotheses presented above are readily testable by additional molecular analyses or by further geographic and longitudinal sampling of type $b$ populations.

\section{Hypothesis for the Global Population Stucture and Molecular Epidemiology of Serotype b Organisms}

Can the data generated be used to develop a global hypothesis for serotype $\mathrm{b} H$. influenzae epidemiology and evolution? Two questions are of special interest. Why is the type b population in the United States different in clonal composition and more diverse genetically than populations in northwestern Europe, Papua New Guinea, and certain other areas? Is there a causal relationship between the degree of ethnic or racial mixing of human populations and the extent of clonal diversity in bacterial populations? This question has received little consideration by medical microbiologists, a circumstance that is 
but one aspect of a more general neglect of evolutionary theory by microbiologists [92].

For many species of pathogenic bacteria, including encapsulated $H$. influenzae, the population structure is basically clonal and the number of clones occurring in appreciable frequencies is small, being on the order of only hundreds, even in E. coli and other abundant species that are highly variable at the level of the individual gene locus. Aparently it is in major part the rarity of chromosomal recombination in conjunction with the periodic purging of variation through selection of fitness mutants that severely limits the number of multilocus gene combinations (clones) of serotype b $H$. influenzae, $E$. coli, and other bacteria, which would otherwise be astronomical [93]. With asexuality or very low rates of recombination, the appropriate primary unit of genetic analysis is the multilocus genotype or clone, rather than the individual gene; the units of differentiation of older genetic structures may be preserved even when there is extensive migration and mixing of human and bacterial populations. Moreover, the evolutionary information content of clones is much greater than that of alleles, since convergence to multilocus genotypes is extremely unlikely, whereas convergence to the same electromorph (allele) is a real possibility.

The four phylogenetic lineages of clones of serotype b isolates - A1; A2-A5; B1, B3-C1; and J1-J3 (figure 1) - are highly distinctive in multilocus genotype, which means that their phylogenetic relationships are relatively deep. Hence, the pattern of global geographic variation in clonal composition of populations discovered for serotype $\mathrm{b} H$. influenzae does not represent merely a transitional state in the relatively recent spread of new minor mutant strains. Because studies of the Enterobacteriaceae indicate that nucleotide substitutions in the coding regions of bacterial genomes accumulate at a more or less constant rate of $1 \%$ per million years [94], this degree of genetic differentiation indicates that the several phylogenetic lineages expressing the type $b$ capsule have had relatively long histories of differentiation; they are old.

It is suggested that the occurrence of greater clonal diversity in populations of serotype $b$ isolates in the United States than those in northwestern Europe and Papua New Guinea reflects the much greater racial and ethnic admixture of the North American human populations. According to this hypothesis, a large component of the geographic variation in clonal composition of serotype $\mathrm{b} H$. influenzae seen today reflects an older pattern of differentiation that evolved in relative geographic isolation before the Age of Exploration (beginning about 450 years ago) and has not yet been completely obscured by recent demogra hic changes. Since then some clones that were formerly confined to certain continents and segments of the human species, especially those of European and African origin, became worldwide or nearly so in distribution. In this category are placed clones of ET 1.9 and certain allied cell lines. Similarly, ET 12.8 probably had its origin in Africa or Asia, and ET 25.6 probably arose in eastern Asia or Oceania.

Two lines of evidence sugggest that ET 1.9 is an old European/Caucasian clone and ET 12.5 is a new clone that has rather recently spread through Europe. First, as discussed above, the frequencies of clones causing invasive disease in Iceland are essentially the inverse of those in mainland Europe: $82 \%$ are ET 1.9 , whereas ET 12.5 accounts for $<10 \%$ of serotype $b$ disease on this island. In addition, on the basis of the geographic distribution of multilocus genotypes in Iceland, there is reason to believe that ET 12.5 has only recently been introduced. On the assumption that ET 1.9 is the old Icelandic strain, we reasoned that it should also occur in appreciable frequency in human populations that share a relatively high degree of ethnicity with primordial or ancient Icelandic populations and that have had relatively little ethnic admixture because of geographic isolation. Such a population exists in the Maritime Provinces of Canada, and strains obtained from a collection of serotype $b$ isolates held by one of us (J. H.) revealed that ET 1.9 and ET 12.5 accounted for $66 \%$ and $22 \%$, respectively, of isolates from Prince Edward Island and New Brunswick. Moreover, 32 of 33 ET 1.9 isolates from Iceland were typed as OMP pattern $2 \mathrm{~L}$ (the exception was an isolate of ET 1.9/OMP $1 \mathrm{H}$ recovered from a 5-year-old American child at the U.S. naval base at Keflavik, Iceland, and all 19 ET 1.9 isolates tested from the Maritime Provinces were also subtype 2L (table 4). In contrast, the ET 1.9/OMP 2L subclone accounted for only $3 \%$ and $17 \%$ of isolates tested from mainland Europe, and non-Maritime provinces of Cana$\mathrm{da}$, respectively, and has never been recovered elsewhere in appreciable frequency.

As an alternative explanation for the observed pattern of geographic variation in clonal composition of serotype $\mathrm{b} H$. influenzae populations, it may be 
suggested that there are, in fact, no old human racial or ethnic correlates of the contemporary global pattern of distribution and abundance of the various clones or clone groups. The major phylogenetic groups of serotype b clones did not evolve in geographic isolation in conjunction with the racial differentiation of humans; rather, all the major clonal groups have since earliest times been present in all major geographic regions and human populations. This hypothesis postulates that the contemporary pattern of variation in the relative abundance of certain clones in northwestern Europe, North America, Papua New Guinea, and elsewhere can be attributed to differential susceptibility to various clones on the part of human populations. Thus, for example, the native Papua New Guineans may have had a long history of exposure to all the major clonal types, and the present predominance of ET 25.6 and closely related clones in Papua New Guinea may reflect a greater susceptibility to clones of that group, as opposed to ET 12.5 or other clones. Susceptibility could be genetically and/or environmentally determined and, as shown by research in genetic epidemiology [95], it would be difficult to distinguish the contributions of these two factors.

A weakness of this hypothesis is that it seems unlikely that all the clones of this organism could have achieved and maintained global distributions while the human species was undergoing geographic differentiation in strong geographic isolation in the past $50,000-100,000$ years $[84,85]$. Because there are no known nonhuman natural hosts of serotype b $H$. influenzae [38], dispersal apparently usually occurs via the close intermingling of children. Strains of type $b$ occur mainly in children from birth to 10 years of age, in whom there is only generally (at most) a $5 \%$ colonization frequency of the throats of healthy individuals not associated with individuals with type $b$ disease [96-99]. (The carriage rate for type b organisms may be much higher in contacts of children with invasive disease [45, 100-102].) Serotype b strains are even rarer in infants (1\% colonization) and in healthy adults ( $<1 \%$ colonization).

Rigorous prospective testing of the two hypotheses is not possible for ethical reasons, but the demonstration that there is a greater chance of finding strongly differentiated clonal types of serotype $b$ $H$. influenzae in the more isolated and genetically differentiated human populations would be more compatible with hypothesis 1 (the postulation of an older, human racial-associated component of bac- terial population structure) than with hypothesis 2 (differential susceptibility of human racial populations). Additionally, hypothesis 1 predicts a stronger correlation between the clonal composition of $H$. influenzae populations and the geographic pattern of human racial differentiation and history of demographic movements than does hypothesis 2 .

The most critical test of the hypotheses comes from an examination of isolates from native or other populations that have experienced very little immigration: hypothesis 1 explicitly predicts that these populations (aboriginals or others) should have a distinctive set of serotype $b$ clones in relatively high frequency, whereas hypothesis 2 does not.

In this respect, six observations, all supporting hypothesis 1, are noteworthy. (1) As discussed elsewhere in this paper, serotype $b$ isolates causing most invasive disease in Iceland, which has had no significant immigration in the last 800 years, are, as a population, genetically quite distinct from strains recovered from invasive episodes in geographically and culturally allied European nations. (2) The clone causing $43 \%$ of the serious type $b$ disease in Papua New Guineans, a geographically, culturally, and genetically divergent and relatively isolated human population, was otherwise rarely encountered in the collection. (3) Sixty percent of invasive isolates from blacks in the Gambia, a group representing an aboriginal African population, were a single clone (ET 12.8); no other single ET accounted for more than $10 \%$ of cases, and isolates of most other ETs were closely allied to those of ET 12.8. Moreover, all six isolates of ET 12.8 tested for OMP pattern were subclone ET 12.8/OMP 2L, whereas only eight of 97 ET 12.8 isolates recovered in other countries represented this subclone $(P<.05)$. (4) Nineteen percent of isolates recovered from invasive episodes in Finland were ET 21.8; in other regions of the world sampled, this clone caused, on average, $<1 \%$ of disease $(P<.01)$. (5) Analysis of 61 serotype $b$ isolates from children in Pakistan with pneumonia and sepsis revealed that a single subclone, ET 25.6/OMP $6 \mathrm{U}$, was responsible for $95 \%$ of cases $[70,71]$. (6) Among Eskimos in Alaska, the analysis revealed cases in which ETs were uniquely confined to individuals living in single geographic regions ([42] and J. I. W., manuscript in preparation). Statistically significant differences in the frequency of occurrence of certain clones in native Alaskans compared to non-native Alaskans [42] have been identified.

In sum, there is extensive geographic variation - 
on an intercontinental scale - in the clonal composition of populations of serotype $b \boldsymbol{H}$. influenzae. The bulk of the data favors the hypothesis that a large component of this variation reflects an older pattern of differentiation that evolved in relative geographic isolation before the Age of Exploration approximately 450 years ago and that has not yet been completely obscured by recent human migration and population admixture. However, it is not possible to completely reject the alternative hypothesis that contemporary patterns of variation in the relative abundance of certain clones reflect differential susceptibility on the part of different human populations. Study of isolates from regions of the world not yet sampled, and especially strains recovered from additional human populations that have experienced little recent admixture, will provide critical data bearing on these hypotheses, as will more extensive analysis, at the nucleotide sequence level, of judiciously selected isolates from the sample described here.

\section{Geographic Variation in Genetic Structure of Pathogenic Bacteria}

Our study of encapsulated strains of $H$. influenzae is among the first to examine geographic variation in genetic structure of a bacterial pathogen on a very large scale in a systematic manner. The analysis revealed at least three distinct levels of geographic variation: intercontinental, intracontinental, and intracountry. For type b strains, the most striking aspect of geographic structure is the variation in frequency of recovery of clones from invasive episodes in patients on different continents; but intracontinental differences in clone recovery may also be extensive. For example, in North America, isolates of ET 100 accounted for $4.5 \%$ of strains from Canada, but this clone was not represented among isolates from invasive or carrier sources in the United States.

We cite two examples of intracountry variation. First, also in Canada, most isolates from the Maritime Provinces were ET 1.9, but isolates of this clone have been recovered in other provinces. Second, in the United States isolates of the ET 1.9/2L subclone are more frequently recovered from patients in Minnesota than in Texas [103]. It very well may be that geographic variation in the structure of type b populations extends to the level of the individual community (microgeographic variation [104]), but we made no attempt to test this hypothesis.

Although many ideas may be advanced to explain the occurrence of geographic variation in type $b$ populations, we favor the notion that much of it is due to a relatively slow dispersal velocity for this pathogen. In this regard it is probably significant that geographic variation recorded in populations of $E$. coli [105], an organism that may survive in the environment and resides in the bowel of virtually every warm-blooded species, is far less dramatic than for type $b$ organisms.

Mayr [104] noted in 1963 that the occurrence of genetic differences among spatially segregated populations of a species is most probably a universal phenomenon in the animal kingdom. Varying levels of geographic structure have been noted for small samples of other bacterial pathogens $[48,69,106]$, and failure to detect it in all species may well be the consequence of investigation of insufficient sample sizes. Like many other aspects of prokaryotic evolution [92], the study of geographic variation and the factors accounting for it (molecular and other) is in its infancy.

\section{Implications for the Control of Serotype b Disease}

The restriction of isolates of most clones to one or a few parts of the world suggests that transmission of serotype $b$ strains and those producing other capsular polysaccharides between geographically separate populations is occurring relatively slowly. Because humans are the only known reservoir of $H$. influenzae [38] and children are the primary carriers of serotype $b$ strains, interpopulation spread must occur largely as a result of close contact between children. The implication of the discovery of the slow spread of clones is that in human populations that are relatively isolated for geographic, cultural, or other reasons, and for which no efficacious vaccine currently exists $[107,108]$, strategies designed to decrease the carrier rate might decrease the incidence of disease.

The hypothesis of slow spread of clones between populations is not incompatible with episodes of rapid turnover of clones or subclones within local $[40,103,109]$ or distant [27] populations.

\section{Utility of the Population Genetic Framework}

One of the strong advantages of population genetic analysis of bacterial pathogens not widely appreciated by medical microbiologists and investigators interested in microbial pathogenesis is that it pro- 
vides ready access to a wealth of genetic theory that can be exploited to provide insights and generate testable hypotheses regarding many aspects of pathogenic organisms. Four examples from studies with $H$. influenzae will suffice.

First, the extensive genetic differentiation of division I and II organisms, which approaches specieslevel divergence, suggests that strains assigned to each division should be quite different in the structure of putative virulence factors and natural history. This has been extensively confirmed by studies of the cap region [22,58], human IgAl protease (iga) gene [110], OMP patterns [20-22, 25], and virulence [22,33] of isolates in each division. Perhaps the best indicator of basic biologic differences between isolates in the two primary divisions is the very infrequent recovery of division II isolates from invasive episodes. Based upon the degree of genetic differentiation, it is very possible that division I and division II serotype $a$ and serotype $b$ isolates differ in global regulation [111] of virulence genes.

Second, population genetic analysis revealed that all serotype $d$ isolates examined are very closely related in overall chromosomal genotype and, thus, anticipated the finding that type $d$ strains collected from worldwide sources over several decades have identical cap region RFLP patterns [58]. Three type $\mathrm{d}$ isolates recently have been shown to have closely similar genomic digest patterns revealed by orthogonal field-alternation gel electrophoresis [112].

Third, the occurrence of strong associations of multilocus enzyme genotypes, cap region RFLP patterns, and OMP subtypes and the recovery of isolates with identical genetic properties in widely separated geographic regions and over a 40-year period indicates that, despite the presence of a well-characterized DNA transformation system, the population structure of encapsulated $H$. influenzae is clonal and presaged a recent report of a lack of genetic competence among multiple independent natural isolates expressing serotype b capsule [59].

Fourth, the extensive knowledge of genetic relationships among the very large number of encapsulated strains described in this report led to the recognition that strains of $H$. influenzae biogroup aegyptius recovered from cases of Brazilian purpuric fever [113], a newly described invasive disease among children in certain rural areas in Brazil, are evolutionarily most closely allied with strains producing serotype c capsule (J. M. M. and R. K. S., "Brazilian Purpuric Fever: Evolutionary Genetic Relation- ships of the Case Clone of Haemophilus influenzae Biogroup aegyptius to Encapsulated Strains of Haemophilus influenzae," submitted).

The population genetic framework has revealed a number of observations regarding the evolutionary genetics of encapsulated $\boldsymbol{H}$. influenzae strains that need explaining at the molecular level. First, by what evolutionary mechanism have serotype $d$ strains arisen, most likely from either a serotype $b$ progenitor strain in cluster B (figure 1)? Second, among serotype $\mathrm{b}$ organisms, why is the $\mathrm{H}$ variant of the heatmodifiable OMP P1 present in strains assigned to divisions I and II? Third, why are strains of ET 21.6 frequently associated with asymptomatic carriage in many different geographic areas and only rarely recovered from invasive disease episodes? Fourth, and perhaps most important from a medical standpoint, why do strains of only nine ETs account for $81 \%$ of all invasive episodes worldwide? Fifth, what processes are responsible for the dramatic geographic variation in population structure among serotype $b$ clones? Examination of these and other questions will provide additional insights into the population biology of $H$. influenzae.

\section{Concluding Comment}

Olyhoek et al. [114] have recently analyzed electrophoretic variation in seven metabolic enzymes and two OMPs in 423 isolates of serogroup A N. meningitidis recovered from 23 epidemics or outbreaks occurring in 38 countries on six continents over a 70year period. Caugant et al. $[89,115]$ have studied genetic diversity and relationships among 650 isolates of eight serogroups of $N$. meningitidis recovered from patients in 20 countries. Therefore, detailed information is now available on an intercontinental scale concerning the molecular population genetics and epidemiology of two other important human pathogenic bacterial species. This study of encapsulated $H$. influenzae is the first to identify possible correlations between contemporary patterns of geographic and temporal variation in clonal composition and diversity of the bacterial population and patterns of ethnic or racial differentiation and historical movement of the host human populations. The data suggest that a causal relation exists between degree of ethnic mixing of human populations and degree of diversity in clonal composition of serotype b $H$. influenzae populations.

Knowledge of the global genetic diversity and 
clonal structure of type $b$ strains will permit prospective assessment of the effect of vaccination on the genotypic population structure of the pathogen and may be important in the formulation of vaccination strategies.

A major goal of bacterial population genetic research is to exploit phylogenetic frameworks to contribute to an understanding of the molecular basis of pathogenesis through the analysis and interpretation of the distribution of proven and putative virulence genes and other factors that may be causally related to pathogenicity [116]. The framework presented here can be utilized to address a variety of questions that remain unresolved in $H$. influenzae research and to generate additional testable hypotheses regarding virulence, pathogenicity, and global epidemiology.

\section{References}

1. Turk DC, May JR. Haemophilus influenzae: its clinical importance. London: English University Press, 1967

2. Sell SH, Wright PF, eds. Haemophilus influenzae: epidemiology, immunology, and prevention of disease. New York: Elsevier/North-Holland, 1982

3. Turk DC. The pathogenicity of Haemophilus influenzae. J Med Microbiol 1984;18:1-16

4. Pittman M. Variation and type specificity in the bacterial species Hemophilus influenzae. J Exp Med 1931;53: 471-92

5. Kilian M. A taxonomic study of the genus Haemophilus, with the proposal of a new species. J Gen Microbiol 1976;93:9-62

6. Anderson $\mathrm{P}$, Flesher A, Shaw S, Harding AL, Smith DH. Phenotypic and genetic variation in the susceptibility of Haemophilus influenzae type $b$ to antibodies to somatic antigens. J Clin Invest 1980;65:885-91

7. Loeb MR, Smith DH. Outer membrane protein composition in disease isolates of Haemophilus influenzae: pathogenic and epidemiological implications. Infect Immun 1980;30:709-17

8. Barenkamp SJ, Munson RS Jr., Granoff DM. Subtyping isolates of Haemophilus influenzae type b by outermembrane protein profiles. J Infect Dis 1981;143:668-76

9. van Alphen L, Riemens T, Poolman J, Hopman C, Zanen HC. Homogeneity of cell envelope protein subtypes, lipopolysaccharide serotypes, and biotypes among Haemophilus influenzae type b from patients with meningitis in the Netherlands. J Infect Dis 1983;148:75-81

10. van Alphen L, Riemens T, Poolman J, Zanen HC. Characteristics of major outer membrane proteins of Haemophilus influenzae. J Bacteriol 1983;155:878-85

11. Inzana TJ. Electrophoretic heterogeneity and interstrain variation of the lipopolysaccharide of Haemophilus influenzae. J Infect Dis 1983;148:492-9

12. Inzana TJ, Pichichero ME. Lipopolysaccharide subtypes of
Haemophilus influenzae type b from an outbreak of invasive disease. J Clin Microbiol 1984;20:145-50

13. Gulig PA, Frisch CF, Hansen EJ. A set of two monoclonal antibodies specific for the cell surface-exposed $39 \mathrm{~K}$ major outer membrane protein of Haemophilus influenzae type $b$ defines all strains of this pathogen. Infect Immun 1983;42:516-24

14. Gulig PA, Hansen EJ. Coprecipitation of lipopolysacchride and the 39,000-molecular-weight major outer membrane protein of Haemophilus influenzae type b by lipopolysaccharide-directed monoclonal antibody. Infect Immun 1985;49:819-27

15. Kilian M, Sørensen I, Frederiksen W. Biochemical characteristics of 130 recent isolates from Haemophilus influenzae meningitis. J Clin Microbiol 1979;9:409-12

16. Tiller F-W. Biochemical differentiation of Haemophilus influenzae. Additional characterization of biotypes by carbohydrate fermentation patterns. Zentralbl Bakteriol Mikrobiol Hyg [A] 1982;253:236-46

17. Tebbutt GM. A chemotyping scheme for clinical isolates of Haemophilus influenzae. J Med Microbiol 1984;17:335-45

18. Hampton CM, Barenkamp SJ, Granoff DM. Comparison of outer membrane protein subtypes of Haemophilus influenzae type $b$ isolates from healthy children in the general population and from diseased patients. J Clin Microbiol 1983;18:596-600

19. Granoff DM, Barenkamp SJ, Munson RS Jr. Outer membrane protein subtypes for epidemiologic investigation of Haemophilus influenzae type b disease. In: Sell SH, Wright PF, eds. Haemophilus influenzae: epidemiology, immunology, and prevention of disease. New York: Elsevier/North-Holland, 1982:43-55

20. Musser JM, Granoff DM, Pattison PE, Selander RK. A population genetic framework for the study of invasive diseases caused by serotype b strains of Haemophilus influenzae. Proc Natl Acad Sci USA 1985;82:5078-82

21. Musser JM, Kroll JS, Moxon ER, Selander RK. Clonal population structure of encapsulated Haemophilus influenzae. Infect Immun 1988;56:1837-45

22. Musser JM, Kroll JS, Moxon ER, Selander RK. Evolutionary genetics of the encapsulated strains of Haemophilus influenzae. Proc Natl Acad Sci USA 1988;85:7758-62

23. van Alphen L, Geelen L, Jónsdóttir K, Takala AK, Kayhty H, Zanen HC. Distinct geographic distribution of subtypes of Haemophilus influenzae type b in western Europe. J Infect Dis 1987;156:216-8

24. Allan I, Loeb MR, Moxon ER. Limited genetic diversity of Haemophilus influenzae (type b). Microb Pathog 1987;2:139-45

25. Allan I, Kroll JS, Dhir A, Moxon ER. Haemophilus influenzae serotype a: outer membrane protein classification and correlation with DNA polymorphism at the cap locus. Infect Immun 1988;56:529-31

26. Hoiseth SK, Connelly CJ, Moxon ER. Genetics of spontaneous, high-frequency loss of b capsule expression in Haemophilus influenzae. Infect Immun 1985;49:389-95

27. Barenkamp SJ, Granoff DM, Pittman M. Outer membrane protein subtypes and biotypes of Haemophilus influenzae type b: relation between strains isolated in 1934-1954 and 1977-1980. J Infect Dis 1983;148:1127 
28. Campos J, Garcia-Tornel S, Musser JM, Selander RK, Smith AL. Molecular epidemiology of multiply resistant Haemophilus influenzae type $\mathrm{b}$ in day care centers. J Infect Dis 1987;156:483-9

29. Musser JM, Barenkamp SJ, Granoff DM, Selander RK. Genetic relationships of serologically nontypable and serotype b strains of Haemophilus influenzae. Infect Immun 1986;52:183-91

30. Selander RK, Caugant DA, Ochman H, Musser JM, Gilmour MN, Whittam TS. Methods of multilocus enzyme electrophoresis for bacterial population genetics and systematics. Appl Environ Microbiol 1986;51:873-4

31. Laemmli UK. Cleavage of structural proteins during the assembly of the head of bacteriophage T4. Nature 1970; 227:680-5

32. Lugtenberg B, Meijers J, Peters R, van der Hoek P, van Alphen L. Electrophoretic resolution of the 'major outer membrane protein' of Escherichia coli $\mathrm{K} 12$ into four bands. FEBS Lett 1975;58:254-8

33. Kroll JS, Moxon ER. Capsulation and gene copy number at the cap locus of Haemophilus influenzae type b. J Bacteriol 1988;170:859-64

34. Nei M. Molecular evolutionary genetics. New York: Columbia University Press, 1987

35. Sneath PHA, Sokal RR. Numerical taxonomy: the principles and practice of numerical classification. San Francisco: Freeman, 1973

36. Catlin BW, Bendler JW III, Goodgal SH. The type b capsulation locus of Haemophilus influenzae: map location and size. J Gen Microbiol 1972;70:411-22

37. Kroll JS, Hopkins I, Moxon ER. Capsule loss in H. influenzae type b occurs by recombination-mediated disruption of a gene essential for polysaccharide export. Cell 1988;53:347-56

38. Kilian M, Biberstein EL. Genus II. Haemophilus Winslow, Broadhurst, Buchanan, Krumwiede, Rogers, and Smith 1917. In: Kreig NR, Holt JG, eds. Bergey's manual of systematic bacteriology. Vol. 1. Baltimore: Williams and Wilkins, 1984:558-69

39. Takala AK, Musser JM, Selander RK. High prevalence of Haemophilus influenzae serotype $b$ of a rare electrophoretic type 21 among invasive isolates in Finland [abstract no. 786]. In: Programs and abstracts of the 27th Interscience Conference on Antimicrobial Agents and Chemotherapy. Washington DC: American Society for Microbiology, 1987

40. Takala AK, van Alphen L, Musser JM, Geelen L, Selander RK, Eskola J, Makela PH. Bacteriologic epidemiology of Hemophilus influenzae type b strains causing invasive infections in Finland. $J$ Infect Dis 1989;160:237-42

41. Ward JI, Lum MKW, Hall DB, Silimperi DR, Bender TR. Invasive Haemophilus influenzae type b disease in Alaska: background epidemiology for a vaccine efficacy trial. J Infect Dis 1986;153:17-26

42. Ward J, Burkart K, Christenson P, Fitzgerald M, Musser $J$, Selander R. Outer membrane protein (OMP) and isoenzyme analysis of Haemophilus influenzae type B (Hib) strains in Alaska [abstract no. 785]. In: Programs and abstracts of the 27th Interscience Conference on An- timicrobial Agents and Chemotherapy. Washington, DC: American Society for Microbiology, 1987

43. Losonsky GA, Santosham M, Sehgal VM, Zwahlen A, Moxon ER. Haemophilus influenzae disease in the White Mountain Apaches: molecular epidemiology of a high risk population. Pediatr Infect Dis 1984;3:539-47

44. Slatkin M. Rare alleles as indicators of gene flow. Evolution 1985;39:53-65

45. Ochman H, Whittam TS, Caugant DA, Selander RK. Enzyme polymorphism and genetic population structure in Escherichia coli and Shigella. J Gen Microbiol 1983; 129:2715-26

46. Selander RK, McKinney RM, Whittam TS, Bibb WF, Brenner DJ, Nolte FS, Pattison PE. Genetic structure of populations of Legionella pneumophila. J Bacteriol 1985; 163:1021-37

47. Brenner DJ, Steigerwalt AG, Epple $P$, Bibb WF, McKinney RM, Starnes RWM, Colville JM, Selander RK, Edelstein PH, Moss CW. Legionella pneumophila serogroup Lansing 3 isolated from a patient with fatal pneumonia, and descriptions of $L$. pneumophila subsp. pneumophila subsp. nov., $L$. pneumophila subsp. fraseri subsp. nov., and $L$. pneumophila subsp. pascullei subsp. nov. J Clin Microbiol 1988;26:1695-1703

48. Musser JM, Hewlett EL, Peppler MS, Selander RK. Genetic diversity and relationships in populations of Bordetella spp. J Bacteriol 1986;166:230-7

49. Yamada Y, Akita M. An electrophoretic comparison of enzymes in strains of Gluconobacter species. J Gen AppI Microbiol 1984;30:115-26

50. Gilmour MN, Whittam TS, Kilian M, Selander RK. Genetic relationships among the oral streptococci. J Bacteriol 1987;169:5247-57

51. Chun PK, Sensabaugh GF, Vedros NA. Genetic relationships among Neisseria species assessed by comparative enzyme electrophoresis. J Gen Microbiol 1985;131:3105-15

52. Selander RK, Musser JM, Caugant DA, Gilmour MN, Whittam TS. Population genetics of pathogenic bacteria. Microb Pathog 1987;3:1-7

53. Tibayrenc M, Ayala FJ. Isozyme variability in Trypanosoma cruzi, the agent of Chagas' disease: genetical, taxonomical, and epidemiological significance. Evolution 1988; 42:277-92

54. Beverley SM, Ismach RB, Pratt DM. Evolution of the genus Leishmania as revealed by comparisons of nuclear DNA restriction fragment patterns. Proc Natl Acad Sci USA 1987;84:484-8

55. Caccone A, Powell JR. Molecular evolutionary divergence among North American cave crickets. II. DNA-DNA hybridization. Evolution 1987;41:1215-38

56. Eskola J, Peltola H, Takala AK, Käyhty H, Hakulinen $M$, Karanko V, Kela E, Rekola P, Rönnberg P-R, Samuelson JS, Gordon LK, Mäkelä PH. Efficacy of Haemophilus influenzae type b polysaccharide-diphtheria toxoid conjugate vaccine in infancy. N Engl J Med 1987;317:717-22

57. Zwahlen A, Rubin LG, Moxon ER. Contribution of lipopolysaccharide to pathogenicity of Haemophilus influenzae: comparative virulence of genetically-related strains in rats. Microb Pathog 1986;1:465-73 
58. Kroll JS, Zamze S, Loynds B, Moxon ER. Common organization of chromosomal loci for production of different capsular polysaccharides in Haemophilus influenzae. J Bacteriol 1989;171:3343-7

59. Cope L, Edgley H, Hansen E. Lack of genetic competence among strains of Haemophilus influenzae type b [abstract no. D-36]. In: Abstracts of the 89th Annual Meeting of the American Society for Microbiology. Washington, DC: American Society for Microbiology, 1989:88

60. Loeb MR, Woodin KA. Cross-reactivity of surface-exposed epitopes of outer membrane antigens of Haemophilus influenzae type b. Infect Immun 1987;55:2977-83

61. Takala AK, van Alphen L, Eskola J, Palmgren J, Bol P, Mäkelä PH. Haemophilus influenzae type b strains of outer membrane subtypes 1 and lc cause different types of invasive disease. Lancet 1987;2:647-50

62. Selander RK, Caugant DA, Whittam TS. Genetic structure and variation in natural populations of Escherichia coli. In: Neihardt FC, Ingraham JL, Low KB, Magasanik B, Schaechter M, Umbarger HE, eds. Escherichia coli and Salmonella typhimurium: cellular and molecular biology. Vol. 2. Washington, DC: American Society for Microbiology, 1987:1625-48

63. Mendelman PM, Syriopoulou VP, Gandy SL, Ward JI, Smith AL. Molecular epidemiology of plasmid-mediated ampicillin resistance in Haemophilus influenzae type b isolates from Alaska. J Infect Dis 1985;151:1061-72

64. Willard JE, Johnson EJ, Daum RS. Interrelationships between physical state, phenotypic stability and transferability of $\beta$-lactamase genes in Haemophilus influenzae. J Gen Microbiol 1982;128:2353-60

65. Campos J, Garcia-Tornel S, Musser JM, Selander RK, Smith AL. Molecular epidemiology of multiply resistant Haemophilus influenzae type $\mathrm{b}$ in day care centers. J Infect Dis 1987;156:483-9

66. Simasathien S, Duangmani C, Echeverria P. Haemophilus influenzae type $\mathrm{b}$ resistant to ampicillin and chloramphenicol in an orphanage in Thailand. Lancet 1980;2:1214-7

67. O'Brien TF, Pla MP, Mayer KH, Kishi H, Gilleece E, Syvanen M, Hopkins JD. Intercontinental spread of a new antibiotic resistance gene on an epidemic plasmid. Science 1985;230:87-8

68. Musser JM, Bemis DA, Ishikawa H, Selander RK. Clonal diversity and host distribution in Bordetella bronchiseptica. J Bacteriol 1987;169:2793-803

69. Beltran P, Musser JM, Helmuth R, Farmer JJ III, Frerichs WM, Waschsmuth IK, Ferris K, McWhorter AC, Wells JG, Cravioto A, Selander RK. Toward a population genetic analysis of Salmonella: genetic diversity and relationships among strains of serotypes $S$. choleraesuis, S. derby, S. dublin, S. enteritidis, S. heidelberg, S. infantis, S. newport, and S. typhimurium. Proc Natl Acad Sci USA 1988;85:7753-7

70. Ghafoor A, Weinberg GA, Ishaq Z, Anwar F, Khalid Nomani N, Kabeer M, Musser JM, Granoff DM. An epidemic of bacteremic nontypable Haemophilus influenzae (NTHI) respiratory infection (ARI) in children in Pakistan [abstract no. 895]. In: Programs and abstracts of the 28th Intersciences Conference on Antimicrobial Agents and Chemotherapy. Washington DC: American Society for Microbiology, 1988
71. Weinberg GA, Ghafoor A, Ishaq Z, Nomani NK, Kabeer M, Anwar F, Burney MI, Qureshi AW, Musser JM, Selander JM, Granoff DM. Clonal analysis of Hemophilus influenzae strains isolated from children from Pakistan with lower respiratory tract infections. J Infect Dis 1989;160:634-43

72. Munson RS Jr, Kabeer MH, Lenoir AA, Granoff DM. Epidemiology and prospects for prevention of disease due to Haemophilus influenzae in developing countries. Rev Infect Dis 1989;11(Suppl):S588-97

73. Bjarnason O, Bjarnason V, Edwards JH, Fridriksson S, Magnusson $M$, Mourant AE, Tills $D$. The blood groups of Icelanders. Ann Hum Genet 1973;36:425-58

74. Thompson EA. The Icelandic admixture problem. Ann Hum Genet 1973;37:69-80

75. Cliff A, Haggett P. Island epidemics. Sci Am 1984;250(5): 138-47

76. Kawakami Y, Okimura Y, Kanai M. Biochemical properties and antimicrobial susceptibility of recent clinical isolates of Haemophilus species. Microbiol Immunol 1981;25:79-84

77. Uehara S, Terashima I, Nakamura A, Kurosaki T, Sugaya $\mathrm{N}$, Okimoto Y, Himi K. The role of Haemophilus influen$z a e$ in lower respiratory infections in childhood. Acta Paediatra Jpn 1985;89:94-101

78. Thong ML, Puthucheary SD, Omar A. Haemophilus influenzae meningitis. Asean J Clin Sci 1983;4:47-56

79. Sherry B, Emanuel I, Kronmal RA, Smith AL, Char LF, Gale $\mathrm{JL}$, Walkley $\mathrm{E}$. Interannual variation of the incidence of Haemophilus influenzae type $\mathrm{b}$ meningitis. JAMA 1989;261:1924-9

80. Petersen GM, Silimperi DR, Roter JI, Terasaki PI, Schanfield MS, Park MS, Ward JI. Genetic factors in Haemophilus influenzae type $\mathrm{b}$ disease susceptibility and antibody acquisition. J Pediatr 1987;110:228-33

81. Siber GR, Santosham M, Priehs CM, Reid R, Letson W, Madore D, Eby R. Impaired antibody (ab) response to $H$. influenzae b (Hib) capsular polysaccharide (CP) in Native American children [abstract no. 326]. In: Programs and abstracts of the 27th Interscience Conference on Antimicrobial Agents and Chemotherapy. Washington, DC: American Society for Microbiology, 1987

82. Blackwell CC, Jonsdóttir K, Hanson M, Todd WTA, Chaudhuri AKR, Mathew B, Brettle RP, Weir DM. Nonsecretion of $A B O$ antigens predisposing to infection by Neisseria meningitidis and Streptococcus pneumoniae [letter]. Lancet 1986;2:284-85

83. Blackwell CC, Jonsdottir K, Hanson MF, Weir DM. Nonsecretion of $\mathrm{ABO}$ blood group antigens predisposing to infection by Haemophilus influenzae [letter]. Lancet 1986;2:687

84. Cavalli-Sforza LL, Bodmer WB. The genetics of human populations. San Franciso: W. H. Freeman, 1971

85. Cavalli-Sforza LL, Piazza A, Menozzi P, Mountain J. Reconstruction of human evolution: bringing together genetic, arachaeological, and linguistic data. Proc Natl Acad Sci USA 1988;85:6002-6

86. Kubitschek HE. Operation of selection pressure on microbial populations. In: Carlile MJ, Skehel JJ, eds. Evolution in the microbial world. London: Cambridge University Press, 1974:105-30 
87. Koch AL. The pertinence of the periodic selection phenomenon to prokaryote evolution. Genetics 1974;77:127-42

88. Levin BR. Periodic selection, infectious gene exchange and the genetic structure of $E$. coli populations. Genetics 1981;99:1-23

89. Caugant DA, Frøholm LO, Bøvre K, Holten E, Frasch CE, Mocca LF, Zollinger WD, Selander RK. Intercontinental spread of a genetically distinctive complex of clones of Neisseria meningitidis causing epidemic disease. Proc Natl Acad Sci USA 1986;83:4927-31

90. Palese $P$, Kingsbury DW, eds. Genetics of influenza viruses. New York: Springer-Verlag, 1983

91. Stuart-Harris C, Potter CW. The molecular virology and epidemiology of influenza. London: Academic Press. 1984

92. Woese CR. Bacterial evolution. Microbiol Rev 1987;51:221-71

93. Selander RK, Levin BR. Genetic diversity and structure in Escherichia coli populations. Science 1980;210:545-7

94. Ochman H, Wilson AC. Evolutionary history of enteric bacteria. In: Neihardt FC, Ingraham JL, Low KB, Magasanik B, Schaechter M, Umbarger HE, eds. Escherichia coli and Salmonella typhimurium: cellular and molecular biology. Vol. 2. Washington, DC: American Society for Microbiology, 1987:1649-54

95. Chakraborty R, Szathmary EJE, eds. Progress in clinical and biological research. Vol. 194. Diseases of complex etiology in small populations. Ethnic differences and research approaches. New York: Alan R. Liss, 1985

96. Dawson B, Zinnemann K. Incidence and type distribution of capsulated $H$, influenzae strains. Br Med J 1952;1:740-2

97. Masters PL, Brumfitt W, Mendez RL, Likar M. Bacterial flora of the upper respiratory tract in Paddington families, 1952-4. Br Med J 1958;1:1200-5

98. Turk DC. Naso-pharyngeal carriage of Haemophilus influenzae type b. J Hyg (Lond) 1963;61:247-56

99. Straker EA. Serological typing of meningeal strains of $H$. influenzae. Lancet 1945;1:817

100. Schiffer MS, MacLowry J, Schneerson R, Robbins JB, McReynolds JW, Thomas WJ, Bailey DW, Clarke EJ Jr, Mueller EJ, Escamilla J. Clinical, bacteriological, and immunological characterisation of ampicillin-resistant Hemophilus influenzae type b. Lancet 1974;2:257-9

101. Ward JI, Gorman G, Phillips C, Fraser DW. Haemophilus influenzae type b disease in a day-care center. Report of an outbreak. J Pediatr 1978;92:713-7

102. Yogev R, Lander HB, Davis AT. Effect of TMP-SMX on nasopharyngeal carriage of ampicillin-sensitive and ampicillin-resistant Hemophilus influenzae type b. J Pediatr 1978;93:394-7

103. Granoff DM, Sheetz K, Pandey JP, Nahm MH, Rambeck JH, Jacobs JL, Musser J, Selander RK, Kabeer M, Murphy TV, Osterholm MT. Host and bacterial factors associated with Haemophilus influenzae type b disease in
Minnesota children vaccinated with type b polysaccharide vaccine. J Infect Dis 1989;159:908-16

104. Mayr E. Animal species and evolution. Cambridge, Mass: Belknap Press of Harvard University Press, 1963

105. Whittam TS, Ochman H, Selander RK. Geographic components of linkage disequilibrium in natural populations of Escherichia coli. Mol Biol Evol 1983;1:67-83

106. Musser JM, Rapp VJ, Selander RK. Clonal diversity in Haemophilus pleuropneumoniae. Infect Immun 1987;55: 1207-15

107. Gilsdorf JR. Bacterial meningitis in southwestern Alaska. Am J Epidemiol 1977;106:388-91

108. Ward JI, Brenneman G, Letson G, Heyward W, the Alaska Vaccine Efficacy Trial Study Group. Limited protective efficacy of an $H$. influenzae type b conjugate vaccine PRP$D$ in Native Alaskan infants immunized at 2-, 4-, and 6-months of age [abstract no. 1127]. In: Programs and abstracts of the 28th Interscience Conference on Antimicrobial Agents and Chemotherapy. Washington, DC: American Society for Microbiology, 1988

109. Crowe BA, Wall RA, Kusecek B, Neumann B, Olyhoek T, Abdillahi H, Hassan-King M, Greenwood BM, Poolman JT, Achtman M. Clonal and variable properties of Neisseria meningitidis isolated from cases and carriers during and after an epidemic in The Gambia, West Africa. J Infect Dis 1989;159:686-700

110. Poulsen K, Hjorth JP, Kilian M. Limited diversity of the immunoglobulin A1 protease gene (iga) among Haemophilus influenzae serotype b strains. Infect Immun 1988;56:987-92

111. Miller JF, Mekalanos JJ, Falkow S. Coordinate regulation and sensory transduction in the control of bacterial virulence. Science 1989;243:916-22

112. Lee JJ, Smith HO, Redfield RJ. Organization of the Haemophilus influenzae Rd genome. J Bacteriol 1989;171: 3016-24

113. Brazilian Purpuric Fever Study Group. Brazilian purpuric fever: epidemic purpura fulminans associated with antecedent purulent conjuctivitis. Lancet 1987;2:757-61

114. Olyhoek T, Crowe BA, Achtman M. Clonal population structure of Neisseria meningitidis serogroup A isolated from epidemics and pandemics between 1915 and 1983. Rev Infect Dis 1987;9:665-92

115. Caugant DA, Mocca LF, Frasch CE, Frøholm LO, Zollinger WD, Selander RK. Genetic structure of Neisseria meningitidis populations in relation to serogroup, serotype, and outer membrane protein pattern. J Bacteriol 1987;169: 2781-92

116. Selander RK, Musser JM. The population genetics of bacterial pathogenesis. In: Iglewski BH, Clark VL, eds. Molecular basis of bacterial pathogenesis. Orlando: Academic Press, 1990 (in press) 